\title{
A High Resolution MCML-based Time-to-Digital Converter Implementation
}

by

\section{Tianshuo Zhao}

\begin{abstract}
A thesis submitted to the
Faculty of Graduate and Postdoctoral Affairs

in partial fulfillment of the requirements for the degree of
\end{abstract}

Master of Applied Science in Electrical Engineering

Ottawa-Carleton Institute for Electrical and Computer Engineering

Department of Electrical Engineering

Carleton University

Ottawa, Ontario

August, 2017

(C) Copyright

Tianshuo Zhao, 2017 
The undersigned hereby recommends to the

Faculty of Graduate and Postdoctoral Affairs acceptance of the thesis

\title{
A High Resolution MCML-based Time-to-Digital Converter Implementation
}

\author{
submitted by Tianshuo Zhao \\ in partial fulfillment of the requirements for the degree of
}

Master of Applied Science in Electrical Engineering

Professor Leonard MacEachern, Thesis Supervisor

Professor Niall Tait, Chair,
Department of Electrical Engineering

Ottawa-Carleton Institute for Electrical and Computer Engineering

Department of Electrical Engineering

Carleton University

August, 2017 


\section{Abstract}

A new high-resolution time-to-digital converter (TDC) architecture based on MOSCurrent-Mode-Logic (MCML) was described. Aiming at high resolution and large dynamic range, the prototype was designed using Virtuoso Cadence Analog Design Environment (ADE) and implemented with $0.13 \mu \mathrm{m}$ CMOS technology. A minimum time domain resolution of $8.24 p S$ and a dynamic range of 9 bits were achieved. The capability of operating with variable resolutions was described. With the novel multistep switchable configuration, the TDC is able to operate with four specific resolutions which are $8.24 p S, 10.83 p S, 12.98 p S$ and $14.3 p S$. Each operation mode corresponds to a different power consumption, which makes the new TDC architecture suitable for being embedded in various systems. For instance, a LIDAR is one of the feasible applications. The new TDC designed in this work can serve as the range detection element and provides millimeter-level $(2.5 \mathrm{~mm}, 3 \mathrm{~mm}, 3.5 \mathrm{~mm}$ and $4 \mathrm{~mm}$ correspond to the four operation modes) ranging precision in a LIDAR system. The design and implementation were verified through simulation results. 


\section{Acknowledgments}

My deepest gratitude goes first and foremost to Professor Leonard MacEachern, my supervisor, for his constant patience, academic support and invaluable advice. With his guidance the research has been a good experience of my academic career.

I would like to express my heartfelt gratitude to my parents, who not only supported me for living in this country, but also comforted me spiritually. Without them this thesis could not have been possible.

Also, my thanks would go to the Department of Electronics, Carleton University and people who have given me their help and advice. Blazenka Power and Anna Lee who have given me their kindness and helped me get through the hardest time. Mr. Nagui Mikhail and Mr. Bruce Scott provided a lot of technical support and time. My academic life could not be so smooth and enjoyable without their help.

I would like to give special thanks to my fiancée, Rongman Gao, for all her support and understanding. 


\section{Contents}

Abstract $\quad$ iii

Acknowledgments $\quad$ iv

Table of Contents $\quad$ v

List of Tables $\quad$ ix

List of Figures $\quad \mathrm{X}$

List of Acronyms $\quad$ XV

List of Symbols $\quad$ xviii

1 Introduction 1

1.1 LIDARs In Autonomous Vehicle Systems . . . . . . . . . . . . . 1

1.2 Time-to-Digital Converter in LIDAR Systems . . . . . . . . . . . . . 3

1.3 Organization of the Thesis . . . . . . . . . . . . . . 5

2 Review of Time-to-Digital Conversion 6

2.1 Introduction . . . . . . . . . . . . . . . . . . 6

2.2 Fundamental Analog Time-to-digital Converter . . . . . . . . . . . . 7

2.3 Basic Digital Delay-Line Based TDC . . . . . . . . . . . . . . . 12 
2.4 Looped Time-to-digital Converter . . . . . . . . . . . . . . . . . . 15

2.5 Gated Ring Oscillator Time-to-Digital Converter . . . . . . . . . . . 17

2.6 Hierarchical Time-to-Digital Converter . . . . . . . . . . . . . . . . . 19

2.7 Vernier Time-to-Digital Converter . . . . . . . . . . . . . 23

2.8 Conclusion . . . . . . . . . . . . . . . . . . 25

3 System Level Design $\quad 27$

3.1 Introduction . . . . . . . . . . . . . . . . . 27

3.2 TDC Module Diagram . . . . . . . . . . . . . . . . . 28

3.3 Fundamental Delay Cell Design . . . . . . . . . . . . . . . . . 29

3.3.1 Basic One-level-MCML Circuit . . . . . . . . . . . . . . 30

3.3.2 MCML Design Parameters . . . . . . . . . . . . . . . 31

3.3.3 An Improved Switchable MCML Delay Cell . . . . . . . . . . 34

3.4 MCML-based TDC Core Design and Improvement . . . . . . . . . . . 34

3.4.1 Basic MCML-buffer-based TDC Core . . . . . . . . . . . . 35

3.4.2 An improved 4-steps Switchable MCML-based TDC core . . . 38

3.5 Peripheral Circuits . . . . . . . . . . . . . . . . 41

3.5.1 Ring Closer ................... 41

3.5.2 Loop Counter . . . . . . . . . . . . . . . . . 41

3.5.3 PISO-Register . . . . . . . . . . . . . . . 43

4 Circuit Design and Implementation 45

4.1 Logic Gate Library Design . . . . . . . . . . . . . . . . 46

4.1 INV1 . . . . . . . . . . . . . . . . . 46

$4.1 .2 \quad$ NAND2D1 . . . . . . . . . . . . . . . . . . 47

$4.1 .3 \quad$ BufferD1 . . . . . . . . . . . . . . . . . . . 49

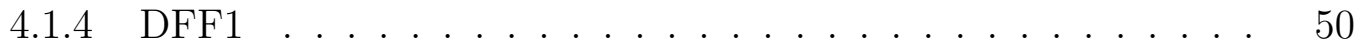


4.1 .5 SWMCML-Buffer . . . . . . . . . . . . . . . . 52

4.2 TDC Implementation . . . . . . . . . . . . . . . . . . 56

4.2.1 Architecture . . . . . . . . . . . . . 56

4.2.2 Fine And Coarse TDC Core Implementation . . . . . . . . . . 58

4.2.3 Ring Closer Implementation . . . . . . . . . . . . . . . . 62

4.2.4 Loop Counter Implementation . . . . . . . . . . . . . . 65

4.2.5 State Freeze Circuit Implementation . . . . . . . . . . . . . . 69

4.2.6 PISO-shift Register Implementation . . . . . . . . . . . . . . . 71

4.3 Output Pad Driver . . . . . . . . . . . . . . . . . . . . 74

4.4 Decoupling Capacitor . . . . . . . . . . . . . . . . 76

4.5 ESD Protection . . . . . . . . . . . . . . . 78

4.6 Chip Micrograph . . . . . . . . . . . . . . . . . . 79

4.7 Chip Layout . . . . . . . . . . . . . . . . . . . . . . . . . . . . . 81

\section{Switchable MCML-Buffer based TDC Simulation Results 83}

5.1 Introduction . . . . . . . . . . . . . . . . . . 83

5.2 Delay Cell Simulated Performance . . . . . . . . . . . . 83

5.2.1 Switchable MCML-Buffer Step Response . . . . . . . . . . . . 83

5.2.2 Corner Simulation of the Delay Cell . . . . . . . . . . . . . . . 87

5.3 TDC Core Simulated Performance . . . . . . . . . . . . . . 89

5.3.1 Calculation of the Loop Delay . . . . . . . . . . . . . . 89

5.3.2 The TDC Performance with 4 Loads Activated . . . . . . . . 91

5.3.3 The TDC Performance with 3 Loads Activated . . . . . . . . 93

5.3.4 The TDC Performance with 2 Loads Activated . . . . . . . . 95

5.3.5 The TDC Performance with 1 Load Activated . . . . . . . . . 97

5.4 Loop Counter Simulation Result . . . . . . . . . . . . . . . . . 99 
5.5 PISO Shift Register and Driving Buffer Chain Simulation Result . . . 101

5.6 Summary of The Simulation Results . . . . . . . . . . . . . 103

6 Conclusions and Future Work 107

6.1 Conclusions . . . . . . . . . . . . . . . . . 107

6.2 Future Work . . . . . . . . . . . . . . . . . . . . 109

$\begin{array}{ll}\text { List of References } & 110\end{array}$ 


\section{List of Tables}

2.1 Comparison of the state-of-the-art . . . . . . . . . . . . . 25

4.1 Inverter INV1 and its corresponding layout. T0 is $3 \mu \mathrm{m}$ wide and 120 $\mathrm{nm}$ long. T1 is $1 \mu \mathrm{m}$ wide and $120 \mathrm{~nm}$ long. . . . . . . . . . . . 47

4.2 The sizes of each transistor in Figure. $4.3 \ldots \ldots \ldots \ldots$

4.3 The sizes of each transistor in Figure $4.5 \ldots \ldots \ldots$

4.4 Pins Descriptions in Figure. $4.9 \ldots \ldots \ldots$

4.5 Pins Descriptions in Figure $4.10 \ldots \ldots \ldots \ldots \ldots$

4.6 Pins Descriptions in Figure. $4.13 \ldots \ldots \ldots$

4.7 Pins Descriptions in Figure $4.16 \ldots \ldots \ldots \ldots$

4.8 Pins Descriptions in Figure. $4.19 \ldots \ldots \ldots \ldots$

4.9 Information of each buffer stage in the driving buffer chain . . . . . 75

5.1 Multi-corner analyses results . . . . . . . . . . . . . . 87

5.2 The recent state-of-the-art versus all modes of the Switchable MCMLBuffer based TDC . . . . . . . . . . . . . . . . . . . . 104 


\section{List of Figures}

1.1 High-level diagram of a LIDAR system showing light source, reflecting object, light receiver, and control and interval measurement sub-systems 3

2.1 Block diagram of basic analog time-to-digital converter. . . . . . . . 8

2.2 Signal diagram for the basic analog time-to-digital converter. . . . . . 8

2.3 Block diagram of an analog TDC based on the pulse stretching technique. 10

2.4 Basic signal diagram of analog time-to-digital converter using the pulse stretching technique. Using this technique, the initial time interval $\Delta T$ is converted into a digital representation. . . . . . . . . . . . . . . 11

2.5 Implementation of a basic delay-line based time-to-digital converter. . 12

2.6 signal diagram of delay-line based TDC. Delayed versions of start event are sampled on the rising edge of the stop signal. The measurement result is represented by a thermometer code at the outputs of the sample elements array. i.e. Flip-Flops or comparators . . . . . . . . . 13

2.7 An example of a looped time-to-digital converter architecture . . . . . 15

2.8 A simplified circuit of GRO-TDC. A fundamental delay cell is implemented with a tri-state inverter. . . . . . . . . . . . . . 17

2.9 An hierarchical time-to-digital converter architecture . . . . . . . . 20

2.10 An improved hierarchical time-to-digital converter architecture with a very short delay-line formed first stage . . . . . . . . . . . . . . 
2.11 Signal diagram of proposed hierarchical improved Time-to-Digital Con-

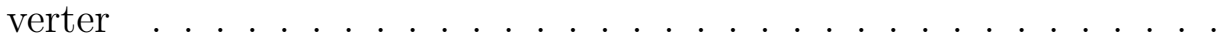

2.12 Cut-out of a Vernier delay-line based TDC based on a first delay-line for the start signal and a second one for the stop signal. The arrival time at two corresponding nodes is assessed by early-late detectors such as flip-flops . . . . . . . . . . . . . . . . .

2.13 Operating principle of Vernier delay-line based TDC. In each stage the chasing signal catches up the leading signal by one $T_{L S B}=t_{d 1}-t_{d 2} \quad . \quad 25$

3.1 Basic block diagram of a time-to-digital converter . . . . . . . . . . 27

3.2 TDC module diagram . . . . . . . . . . . . . . . . . . . . . . . . . 29

3.3 An example of basic MCML circuits. (a)basic MCML inverter/buffer gate. (b)Current steering mechanism of the basic MCML gate . . .

3.4 MCML delay cell analysis. (a) A one-level MCML gate schematic. The highlight portion is approximately modeled in the sub figure below (b) Equivalent small signal model of the hight light portion . . . . . . . . 32

3.5 Diagram of steps switchable MCML-buffer . . . . . . . . . . . . . . 35

3.6 Diagram of the basic MCML-Buffer based TDC core schematic . . . . 36

3.7 Signal diagram of the TDC core schematic in Fig. $3.6 \quad \ldots . . .37$

3.8 Diagram of the improved 4 step switchable MCML-Buffer based TDC

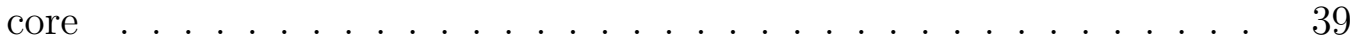

3.9 An example signal of the switchable MCML-Buffer based TDC core . 40

3.10 Diagram of the Ring closer module formed by 2 cross coupled $2 \mathrm{x} 1$ NAND gates . . . . . . . . . . . . . . . . . . . . 42

3.11 Diagram of an 8-bit digital counter . . . . . . . . . . . . . . . . 42

3.12 Diagram of a parallel-in-serial-out shift register . . . . . . . . . . 43 
4.1 NAND2D1 gate and its corresponding layout. The widths of T0 and $\mathrm{T} 1$ is $4 \mu \mathrm{m}$ with 2 fingers and their lengths are $120 \mathrm{~nm}$. T2 and T3 are $3.04 \mu m$ wide with 2 fingers and $120 \mathrm{~nm}$ long. . . . . . . . . . 48

4.2 An example of a Buffer gate and its corresponding layout. The widths of T0 and T3 are $1.04 \mu \mathrm{m}$ and lengths are $120 \mathrm{~nm}$. T1 and T2 are 780 $n m$ wide and $120 \mathrm{~nm}$ long. . . . . . . . . . . . . . . . . . . 49

4.3 The schematic diagram of the D type Flip-Flop. . . . . . . . . . 50

4.4 The layout diagram of the D type Flip-Flop. . . . . . . . . . 51

4.5 The schematic diagram of the four steps switchable MCML Buffer gate 53

4.6 The layout diagram of the 4 steps switchable MCML Buffer gate . . . 54

4.7 The overall block diagram of the switchable TDC design $\ldots \ldots . .57$

4.8 Schematic and layout view of the TDC core . . . . . . . . . 59

4.9 The diagram of the TDC core symbol . . . . . . . . . . . . 60

4.10 Ring Closer Implementation Symbol _ . . . . . . . . . . . . 62

4.11 The schematic of the Ring Closer. This block is made up of two NAND gates. For each NAND one input is for the start signal injection and the other one is for the feed-back signal. . . . . . . . . . . 63

4.12 The layout of the Ring Closer block . . . . . . . . . . . . . . 64

4.13 8-bit rising edge counter hierarchical Symbol . . . . . . . . . . . 65

4.14 The schematic of the Loop Counter. This block is made up of 8 stages. Each stage consists of a Flip-Flop and a logic shifting element. . . . . 67

4.15 The layout of the Loop Counter. All gates are placed in a row. . . . . 68

4.16 The hierarchical symbol of the State Freeze block with all pins . . . . 69

4.17 The schematic view of the State Freeze block . . . . . . . . . . 71

4.18 The layout view of the State Freeze block . . . . . . . . . . 71

4.19 The hierarchical symbol of the PISO shift regesiter $\ldots \ldots \ldots$. . . 72 
4.20 The schematic of the PISO shift regesiter . . . . . . . . . . . 73

4.21 The layout of the PISO shift regesiter . . . . . . . . . . . . . 74

4.22 The schematic of a driving buffer chain . . . . . . . . . . . . 75

4.23 The layout of the driving buffer chain . . . . . . . . . . . . . . 76

4.24 The layout of the PISO shift regesiter . . . . . . . . . . . 76

4.25 Schematic of the ESD protection device and the corresponding layout 79

4.26 The micrograph of the chip. Main components are highlighted. . . . . 80

4.27 Top view of the switchable MCML-Buffer based TDC chip layout. The major building blocks of the novel TDC are a TDC core, counters, PISO-shift registers and driving buffers. The chip margin is $1.6 \mathrm{~mm}$ tall and $1.6 \mathrm{~mm}$ wide. . . . . . . . . . . . . . . . .

5.1 The test bench for the measurement of specific delay for each switched condition. With different control voltage combination (VRP0-VRP3). The MCML-Buffer delay element operates with different propagation delays. . . . . . . . . . . . . . . . . . . .

5.2 Delay cell step response for the four switched conditions, with time delay: TD1 $=8.24 \mathrm{ps}, \mathrm{TD} 2=10.83 \mathrm{ps}, \mathrm{TD} 3=12.98 \mathrm{ps}$, and TD4=14.3 ps

5.3 Step response variations for different temperatures with FF corner simulation. . . . . . . . . . . . . . . . . . . . . 88

5.4 Step response variations for different temperatures with SS corner sim-

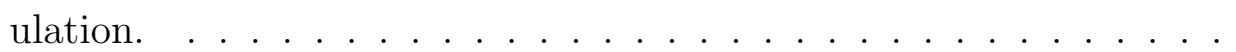

5.5 An average value of the loop delay for the complementary path is equalized. This configuration is able to address the delay asymmetry that is shown in this figure. . . . . . . . . . . . . 90

5.6 The Simulation Result of a $20 \mathrm{~ns}$ Time Interval. The total number of cycles of the upper path is 51 and the lower path is $50 . \ldots . .$. 
5.7 The simulation results of the FFs. A 12-bit thermometer code is acquired. It contains 4 bits of logic 1 followed by 8 bits of logic 0 . . . . 93

5.8 The Simulation Result of a $20 \mathrm{~ns}$ Time Interval. The total number of cycles for the upper path is equal to 35 and the lower path is 36 . . .

5.9 The simulation results of the FFs. A 12-bit thermometer code is acquired. It contains 9 bits of logic 1 followed by 3 bits of logic $0 . \quad$. . . 95

5.10 The Simulation Result of a 20 ns Time Interval. The total number of cycles for the upper path is equal to 30 and the lower path is 29 . . . 96

5.11 The simulation results of the FFs. A 12-bit thermometer code is acquired. It contains 2 bits of logic 1 followed by 10 bits of logic 0 . . .

5.12 The Simulation Result of a 20 ss Time Interval. The total number of cycles for the upper path is equal to 27 and the lower path is $26 \ldots$. . 98

5.13 The simulation results of the FFs. A 12-bit thermometer code is acquired. It contains 7 bits of logic 1 followed by 5 bits of logic $0 . \quad$. . 99

5.14 The simulation result of the Loop Counter. The output binary code is 00100011 (Decimal 35) at the stop event which occurs at $21 \mathrm{~ns} . \quad$. . 100

5.15 The diagram of the test bench. A driving buffer chain is attached. A capacitor and an inductor is used to model the bond pad and the

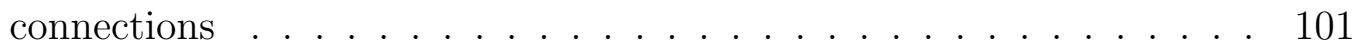

5.16 The simulation result of the PISO Shift Register. . . . . . . . . . . . 102

5.17 Resolution and Power comparison. . . . . . . . . . . . . . . 105 


\section{List of Acronyms}

\section{Acronyms Definition}

\begin{tabular}{ll}
\hline \hline SLAM & Simultaneous Localization And Mapping \\
UGV & Un-manned Ground Vehicle \\
PCL & Point-Cloud Library \\
CMOS & Complementary Metal Oxide Semiconductor \\
CMC & Canadian Micro-electronics Corporation \\
TDC & Time-to-Digital Converter \\
TOF & Time-Of-Flight \\
ADC & Analog-to-Digital Converter \\
DR & Dynamic Range \\
& All-Digital-Phase-Locked-Loop \\
&
\end{tabular}




\begin{tabular}{|c|c|}
\hline MUX & Multiplexer \\
\hline $\mathrm{MCML}$ & MOS-Current-Mode-Logic \\
\hline LPI & Local Passive Interpolation \\
\hline SSP & Single Shot Precision \\
\hline UDSC & Ultra-Deep-Submission-CMOS \\
\hline FI & Fan-In \\
\hline $\mathrm{FO}$ & Fan-Out \\
\hline $\mathrm{FF}$ & Flip-Flop \\
\hline $\mathrm{S} / \mathrm{H}$ & Sample and Hold \\
\hline DNL & Differential Non-Linearity \\
\hline INL & Integrated Non-Linearity \\
\hline PISO & Parallel-In-Serial-Out \\
\hline DETSE & Dual-Edge-Triggered-Storage-Element \\
\hline $\mathrm{DRC}$ & Design-Rule-Checking \\
\hline LVS & Layout-Versus-Schematic \\
\hline TM2B & Thermometer-To-Binary \\
\hline ESD & Electrostatic Discharge \\
\hline ADE & Analog Design Environment \\
\hline
\end{tabular}




\section{List of Symbols}

\section{Symbols Definition}

$\begin{array}{ll}\mathbf{T}_{\mathbf{L S B}} & \text { the minimum time interval that can be resolved by a TDC } \\ p & \text { time amplification factor defines the ratio of the amplification } \\ \mathbf{N} & \text { time interval need to be measured } \\ \varepsilon & \text { the number of stages output HIGH values } \\ \mathbf{B}_{\text {cnt }} & \text { the binary code represents the coarse result } \\ \tau & \text { the resolution of the Looped TDC } \\ \mathbf{M}_{\text {M }} & \text { the number of delay elements that the start event has traversed } \\ \mathbf{B}_{\text {phase }} & \text { measurement result of the fine block in the hierarchical TDC } \\ \mathbf{B}_{\text {FTDC }} & \text { internal calibration coefficient of the improved hierarchical TDC } \\ \kappa & \text { gate oxide capacitance per unit area }\end{array}$




\begin{tabular}{ll}
$C_{g d}$ & gate-to-drain overlap capacitance \\
$C_{d b}$ & drain-to-bulk overlap capacitance \\
$v_{\text {sat }}$ & MOSFET saturation voltage \\
$A_{\text {bulk }}$ & bulk charge effect parameter \\
$T_{l o o p}$ & loop cycle of the MCML-based TDC \\
\hline
\end{tabular}




\section{Chapter 1}

\section{Introduction}

\subsection{LIDARs In Autonomous Vehicle Systems}

Fully autonomous, self-driving vehicles have become a reality over the past several years. With the development of simultaneous localization and mapping (SLAM) technology and the advent of consumer-grade three-dimensional (3D) light detection and ranging (LIDAR) scanners, famous un-manned ground vehicle (UGV) prototypes such as the Google driver-less car are able to travel hundreds of thousands of miles without user control [1].

In order to navigate autonomously, UGV systems require precise localization within a digital 3D map. Rather than using the vehicle's sensors to explicitly perceive lane markings, traffic signs, regional landmarking, etc., metadata is embedded into a prior map, which transforms the difficult perception task into a localization problem. Some existing state-of-the-art methods [2] [3] use reflectivity measurements from 3D LIDAR scanners to create an orthographic map of ground-plane reflectivity. The local digital prior map which is formed by these reflected points is then processed online by point-cloudy library (PCL) technology [4].

LIDAR scanners are combined with high accuracy GPS/INS systems, to enable 
these autonomous vehicles to operation safely and precisely on roadways. However, it is also what is keeping autonomous vehicles from being affordable enough for the average consumer to buy [5]. For instance, the Velodyne 64 channels LIDAR (HDL64e) which has been placed on the top of the famous Google-car costs 75,000 USD. This is higher than the price of a regular luxury car. The cost of LIDAR sensors should drop by 90 percent in the near future [6]. Big companies such as, Tesla Motors Inc, BMW, Ford Motor Co, and Volvo cars have all promised to have fully autonomous cars on the road within five years. 


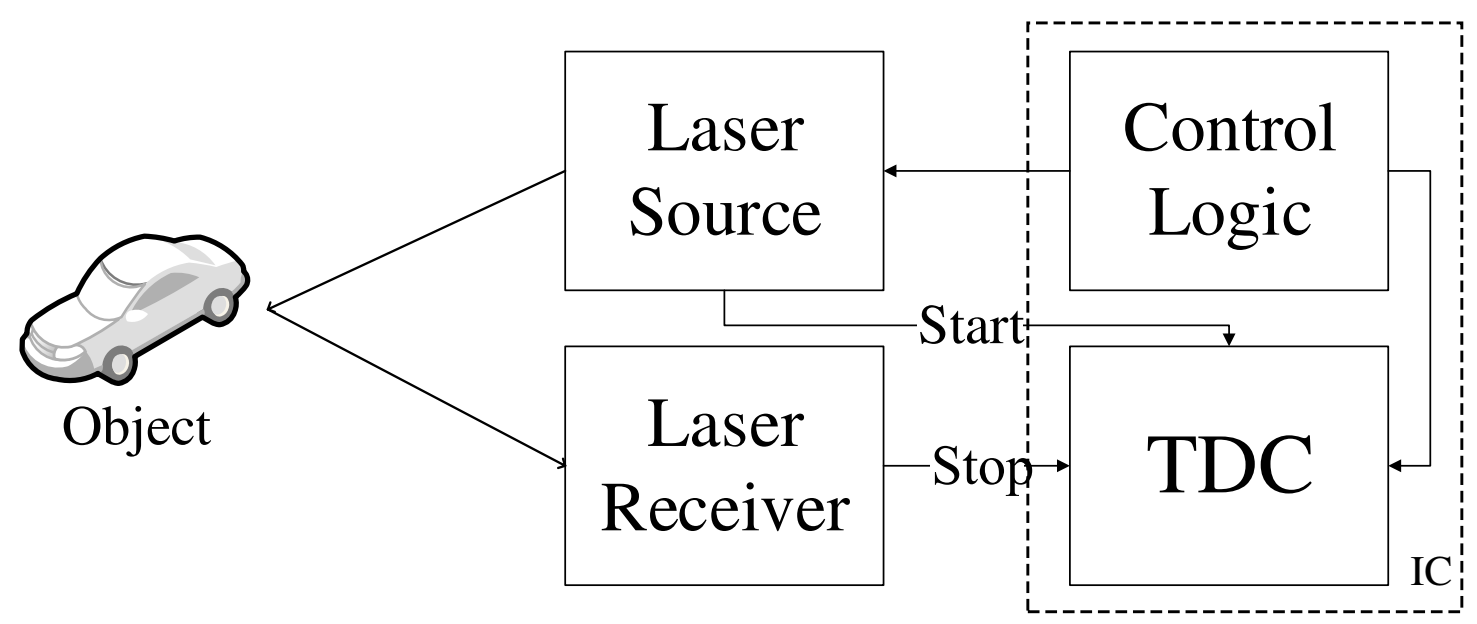

Fig. 1.1: High-level diagram of a LIDAR system showing light source, reflecting object, light receiver, and control and interval measurement sub-systems

\subsection{Time-to-Digital Converter in LIDAR Systems}

Autonomous vehicles use LIDAR scanners extensively, and therefore low-cost and high-performance LIDAR components are desired [5]. A crucial component of any LIDAR system is a time-to-digital converter (TDC), which serves as a phase difference detector, as shown in Figure 1.1.

A LIDAR system typically consists of a laser source channel, a receiving channel, control logic for start and stop timing pulses, and a TDC which serves as the time interval measurement unit. The time interval between an out-going laser pulse directed at a target location and the reflected laser pulse acquired from the target is called the the time-of-flight (TOF) for the laser pulse. The distance to the target location may be computed from the TOF. For Lidars in autonomous vehicles a measurement range of several hundred meters with accuracy on the order of centimeters is desired. [7] These are directly influenced by two TDC performance characteristics: dynamic range and resolution. Usually, a LIDAR scanner requires centimeter-level or even millimeter-level ranging precision. This can be achieved by using a TDC 
with a resolution smaller than tens of picoseconds or even picoseconds. For example, the TDC7200 has a time domain resolution of $55 \mathrm{ps}$ which corresponds to a $1.5 \mathrm{~cm}$ distance resolution in a LIDAR system. Consequently, the measurement capability of a LIDAR system is significantly influenced by TDC key performance parameters. All these crucial characteristics will be presented in the next sub-section. 


\subsection{Organization of the Thesis}

Chapter 2 briefly reviews the existing TDC architectures. By introducing the recent state-of-the-art architectures, a clear track about how to improve the TDC performance will appear. Chapter 3 presents the detailed analysis about MOS-CurrentMode-Logic (MCML) gates and provides a new architecture which makes the TDC resolution tunable. Chapter 4 provides a novel TDC circuit level design and implementation based on a new MCML technique and gives detailed information on how this work improves the key performance characteristics. Results are presented in Chapter 5. Conclusions and future work are presented in Chapter 6. 


\section{Chapter 2}

\section{Review of Time-to-Digital Conversion}

\section{$2.1 \quad$ Introduction}

In this chapter, architectural aspects of time-to-digital converters are discussed in detail. The critical design parameters are high dynamic range, high resolution, high linearity, small die area and low power consumption.

The TDC architectures that have been widely used during the past decade are briefly summarized in the following subsections. Analog time-to-digital converters are the most elegant and well-suited for precise timer design. A loop architecture enables long measurement times with reasonable area and power consumption. For long measurement times a hierarchical TDC system can further reduce the power consumption. A 3rd generation TDC implement with a Vernier architecture achieves sub-gated resolution which is a time domain resolution smaller than a cell propagation delay. Finally, the novel MOS-Current-Mode-Logic based TDC is introduced. A comparison of recent state-of-the-art architectures is summarized in a table as the conclusion of this chapter. 


\subsection{Fundamental Analog Time-to-digital Con- verter}

Time-to-digital converters are essentially a specialized type of analog to digital converter $(\mathrm{ADC})$. Therefore, the first step in time-to-digital conversion is first to convert the time interval into a corresponding voltage. This voltage is then digitized by a conventional ADC. A block diagram is illustrated in Figure 2.1. An example of the signal during a conversion is depicted in Figure 2.2. First, the start and stop event form a pulse with a width corresponding to the time interval to be measured. Next an analog integrator transforms this pulse into a voltage which is then fed to the ADC, which converts it to a digital value.

The dynamic range (DR) of a TDC is defined as the maximum time interval that can measured. There is a fundamental trade-off between this and the maximum number of bits $(\mathrm{N})$ resolvable by the ADC. This is revealed by the following equation $[2$, sec.3.2]:

$$
D R=2^{N} \cdot T_{L S B}
$$

where $T_{L S B}$ is the minimum time interval that can be resolved. From this we can see that the resolution of the ADC is limited by analog constraints. For example, a basic integrator implementation that allows for high-speed is a current source which is connected to an capacitor during the measurement interval. Due to the finite output resistance of the current source the linearity is limited [8]. Generally, a long measurement interval means low resolution and vice versa due to the fact that the capacitance of the integrator is a constant value .

This fundamental TDC is very simple, but it is limited when converting long time 


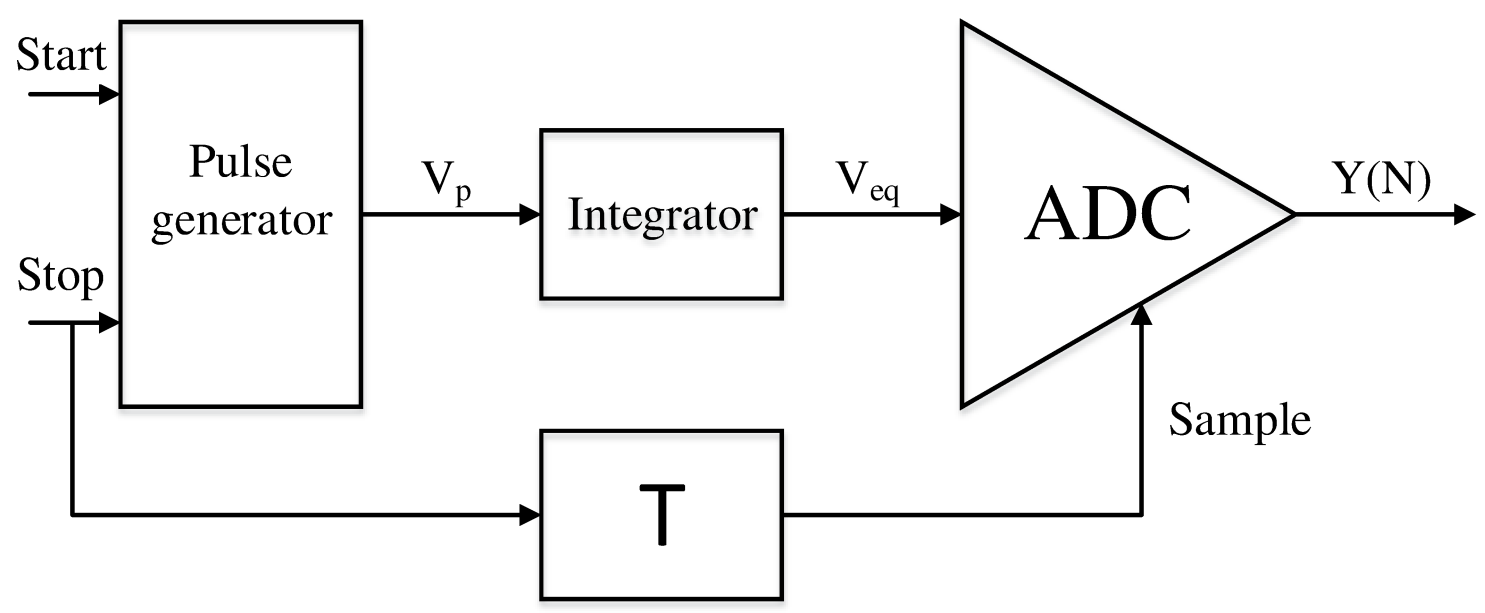

Fig. 2.1: Block diagram of basic analog time-to-digital converter.

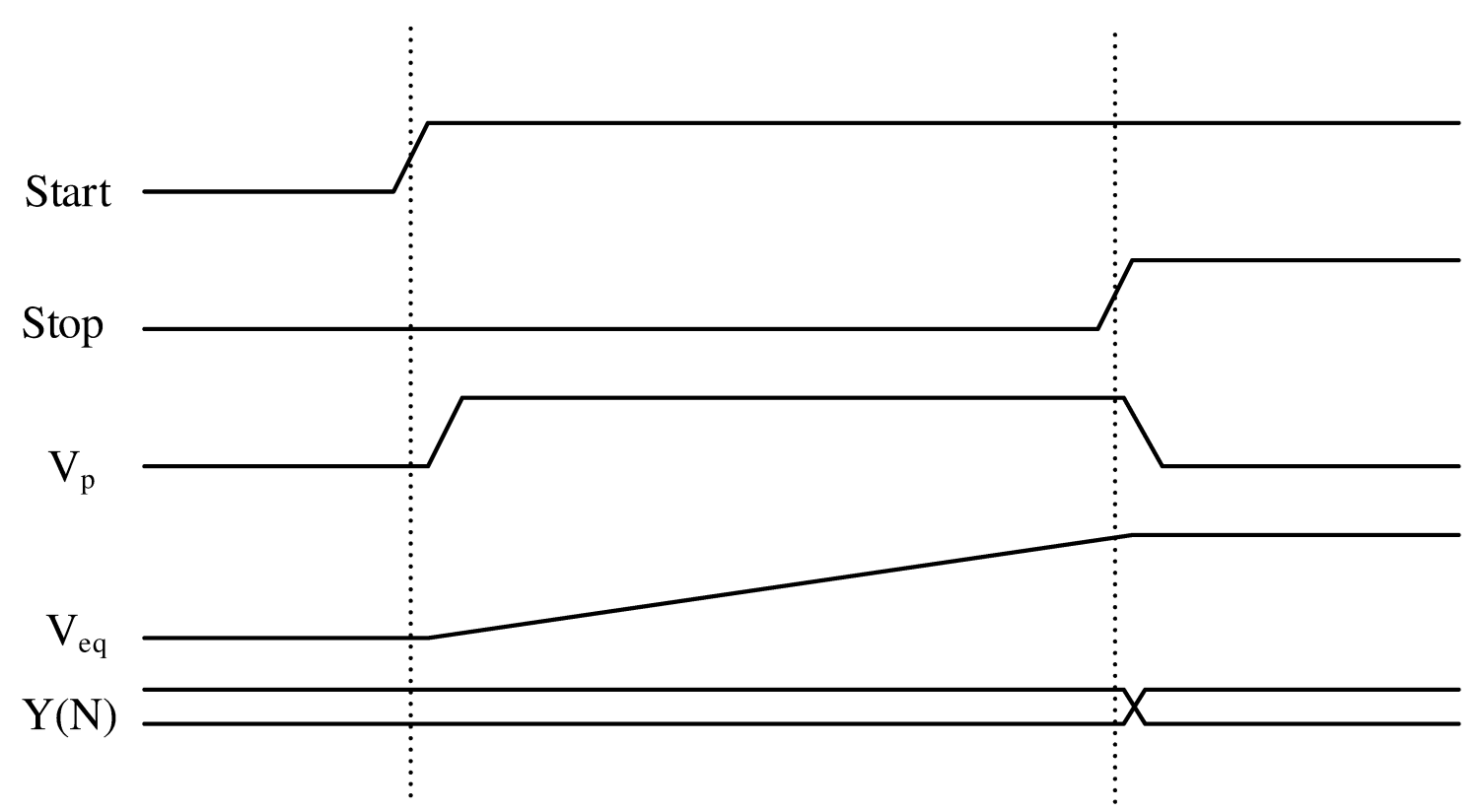

Fig. 2.2: Signal diagram for the basic analog time-to-digital converter.

intervals with a high resolution. The architecture can be improved to overcome this issue allow for measurement at a long time interval.

The way to achieve this is to integrate the signal with both charging and discharging, namely, the "pulse stretching" or "time amplification" technique [9] [10]. An example topology of an analog TDC based on this dual-slope pulse stretching 
method is given in Figure 2.3. As with the previous architecture (Figure 2.1), the input time interval defined by the start and stop signals is integrated. As illustrated in Figure 2.4, on the rising edge of the stop signal, another integrator is activated but with a reduced integration rate $1 / p$, where $p$ is defined as the time amplification factor. A comparator then detects the point in time when the two integrators output the same voltage. Ideally, this happens $(1+p) \Delta T$ seconds after the start event. We can see that the initial time interval $\Delta T$ is stretched by the factor $(1+p)$. Consequently, this approach increases the capability of measuring a relatively long time interval. If the factor $p$ is large enough, the enlarged time interval may be quantized by a simple digital counter.

Although the analog TDCs introduced in this section work with a relatively simple mechanism, there are still several issues that degrade their performance. All the building blocks, i.e. the pulse generator, the integrator, and the ADC, have to meet the full linearity demands of the overall TDC [8]. A basic integrator is usually implemented with a large capacitor charged by a current source. This consumes a large chip area and also limits the conversion speed. Also, the resolution $T_{L S B}$ is strictly limited by the back-end ADC. This makes the optimization of this architecture challenging.

The analog TDC architecture discussed above would be good enough for the purpose of a precise timer design. But TDCs should not just focus on being a time counting device. TDCs have been used in many applications: for example, they are used as the phase difference detector in all-digital-phase-locked-loop (ADPLL), which is a crucial building block in the wireless communication field. They are also used as the range measurement core for the LIDAR system in the modern remote sensing field [11]. The advantages of time domain signal processing and the superior scaling properties of TDCs are commonly known [8]. However, this does not hold for any 


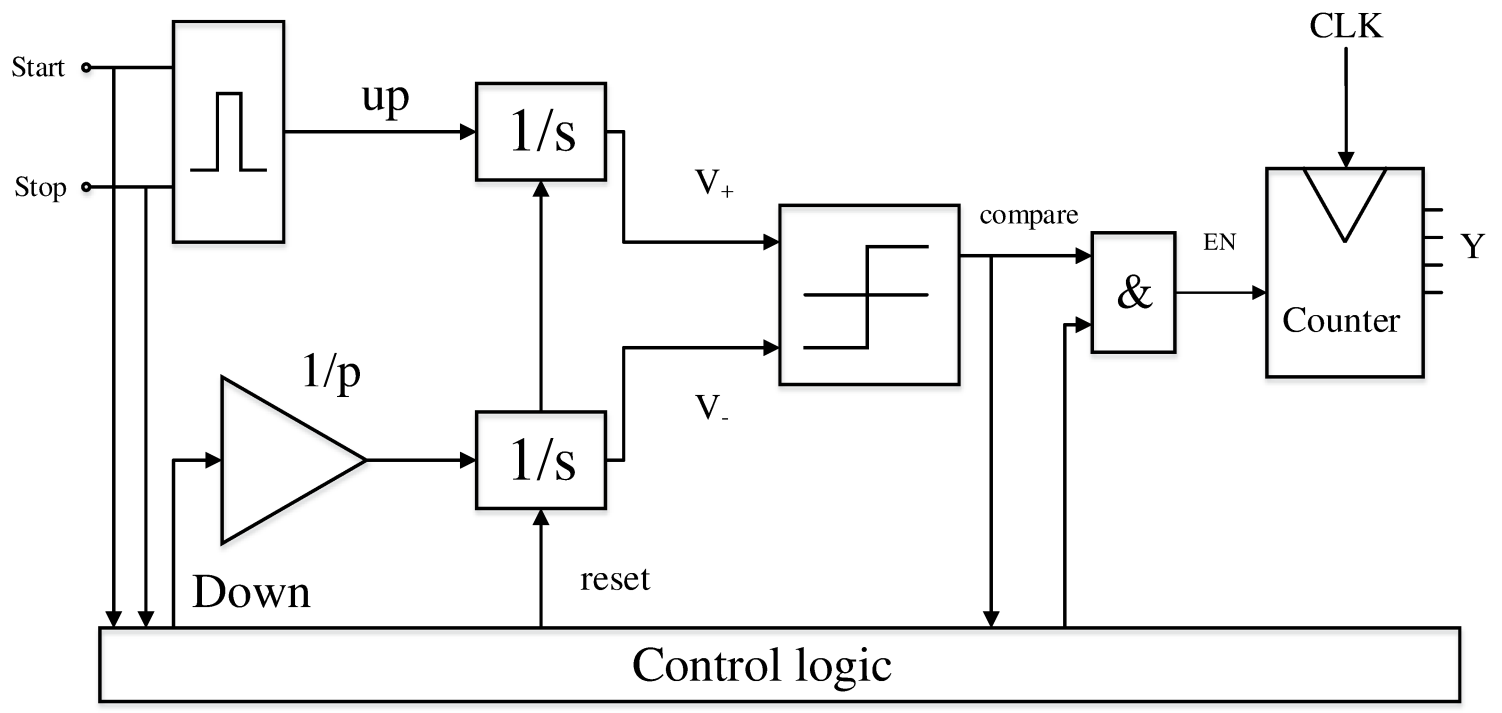

Fig. 2.3: Block diagram of an analog TDC based on the pulse stretching technique.

analog TDC which converts time domain information first into the analog domain and then to the digital domain. The TDC can only benefit from all the properties of sub-micron CMOS technologies if there is no analog conversion required. This leads to TDCs that are implemented with delay cells and counter based architectures. These are known as 2nd generation TDCs. A few most widely used 2nd generation TDCs will be discussed next. 


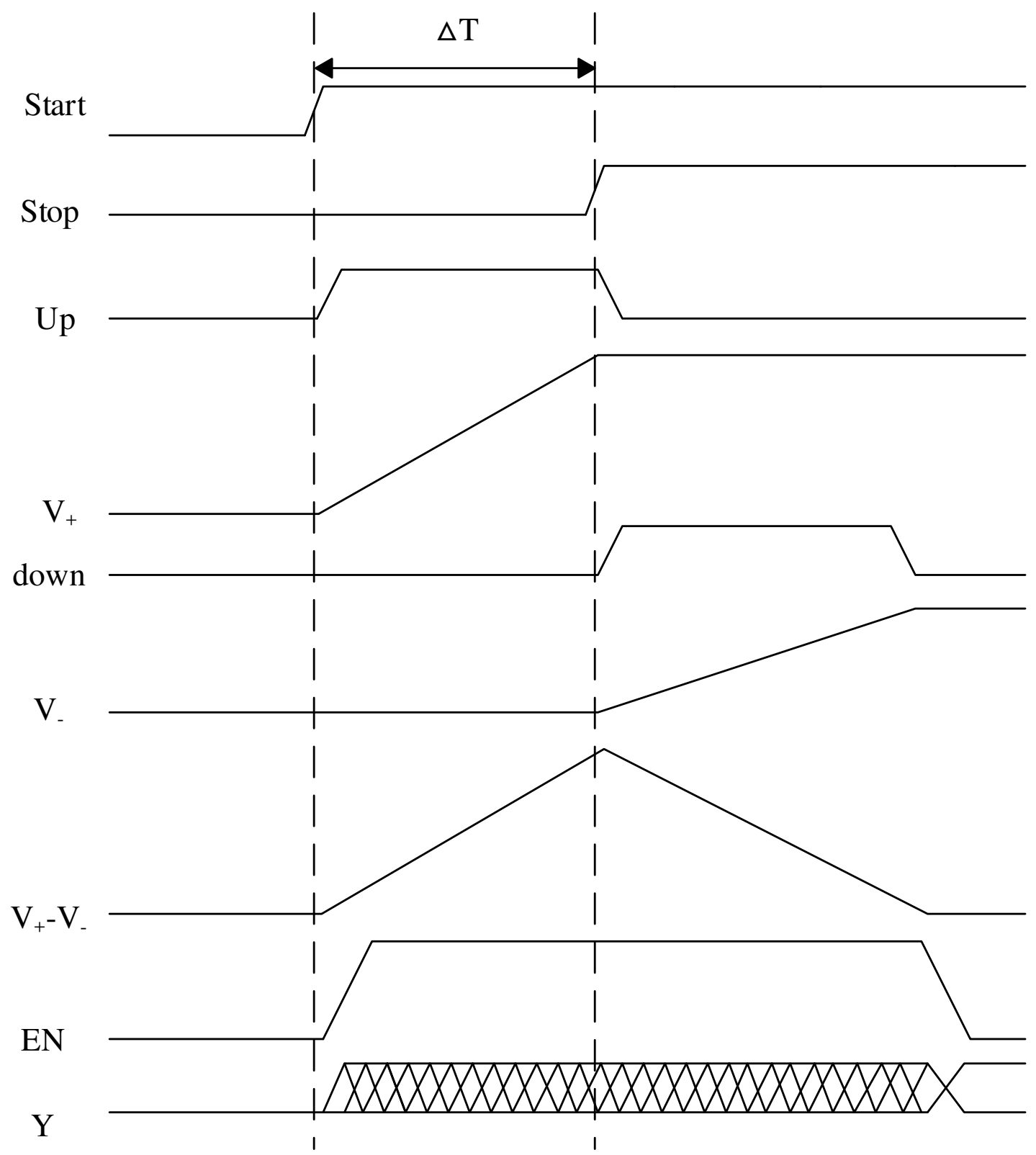

Fig. 2.4: Basic signal diagram of analog time-to-digital converter using the pulse stretching technique. Using this technique, the initial time interval $\Delta T$ is converted into a digital representation. 


\subsection{Basic Digital Delay-Line Based TDC}

As discussed in the previous section, TDCs implemented by sub-micron CMOS technologies benefit from all the advantages of digital signal processing. One of the basic approaches to achieve this is delay-line based time-to-digital conversion. An implementation of basic delay-line based TDC is depicted in Figure 2.5.

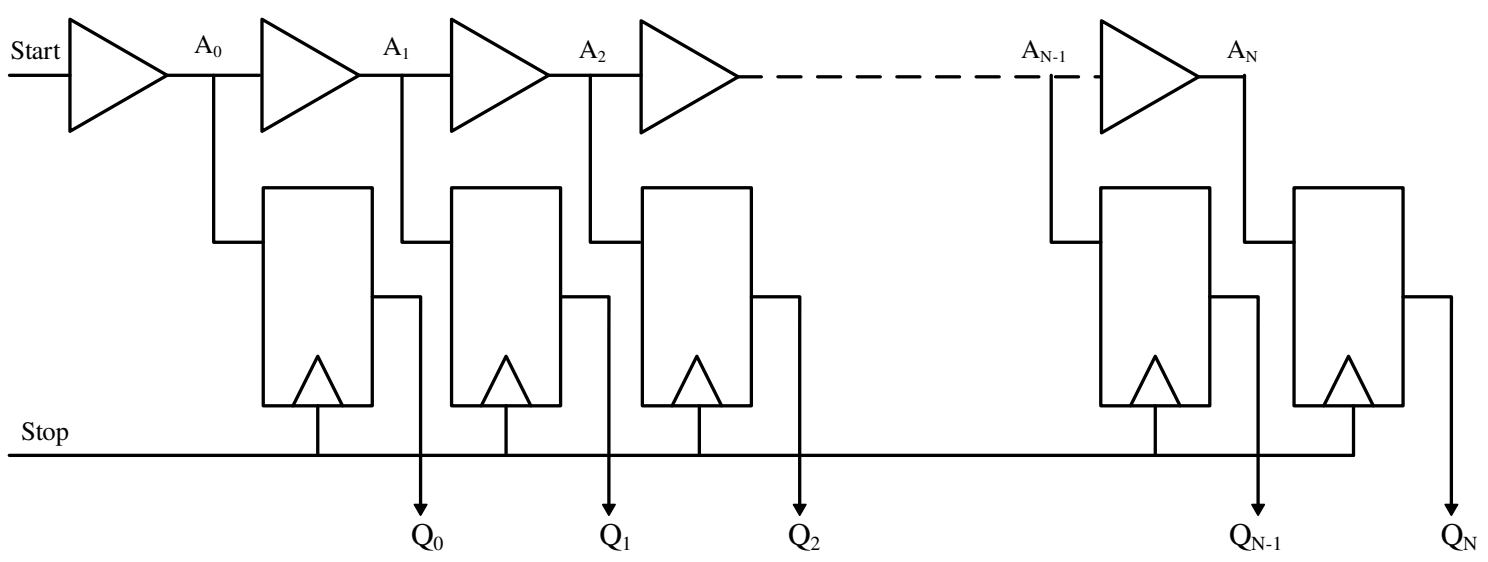

Fig. 2.5: Implementation of a basic delay-line based time-to-digital converter.

The delay-line TDC works as follows. First, the start signal propagates along the buffer formed delay-line in order to create multiple delayed versions of it. After a certain amount of time, the stop signal triggers. At the rising edge of the stop signal, the sample array which is formed by flip-flops or comparators is activated. The time interval between the start and stop event is exactly the time interval which needs to be measured.

As shown in Figure 2.6, the delayed versions of the start signal, start ${ }_{i}$, are sampled in parallel. The sample process freezes the state of the delay-line at the instant the stop signal occurs. This results in a thermometer code because all the delay stages which have already been passed by the start signal give a HIGH value (logic 1) at the output of the sample elements, and all of the delay stages which have not been 


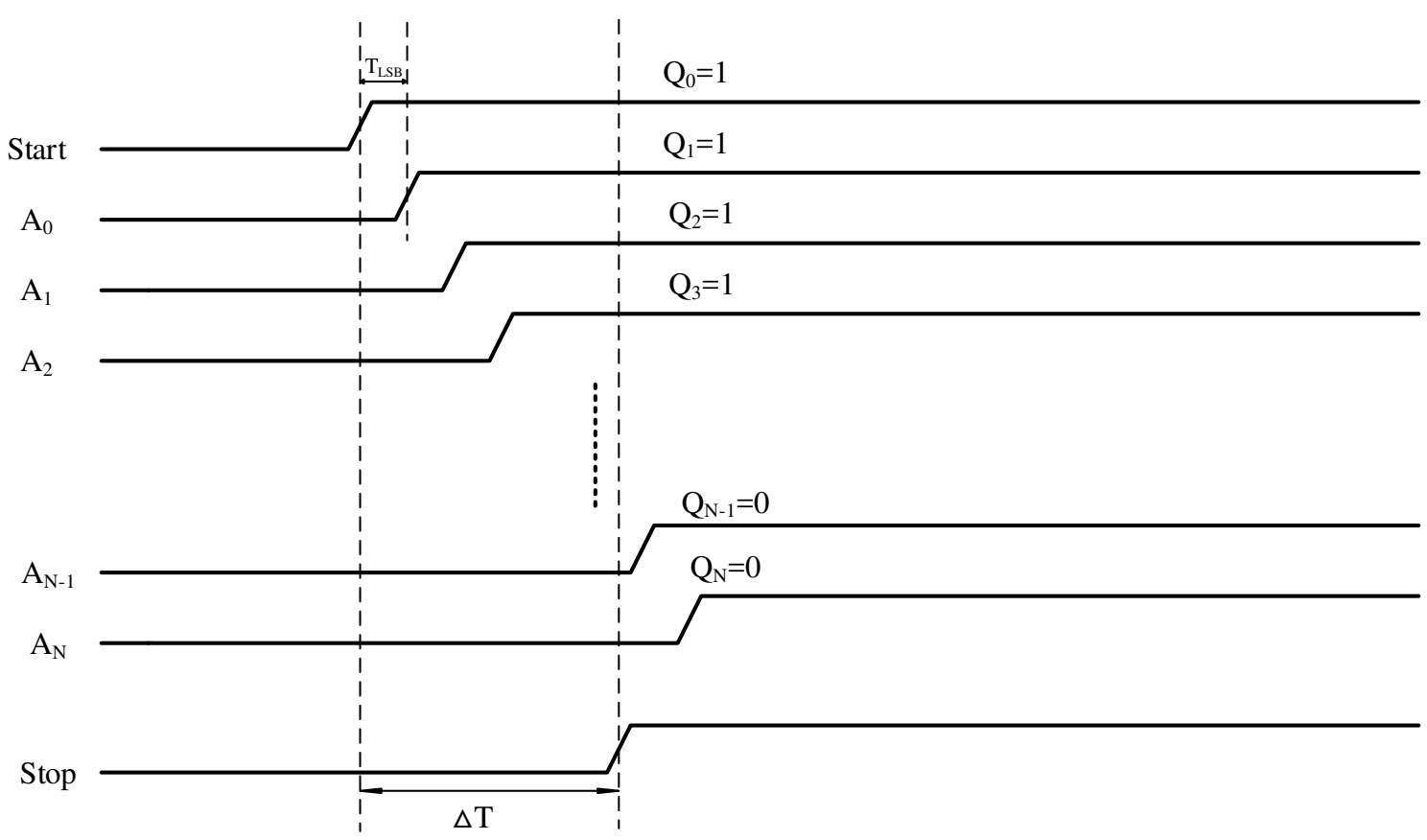

Fig. 2.6: signal diagram of delay-line based TDC. Delayed versions of start event are sampled on the rising edge of the stop signal. The measurement result is represented by a thermometer code at the outputs of the sample elements array. i.e. Flip-Flops or comparators

passed by the start signal yet give a LOW value (logic 0). All of these HIGH and LOW values in sequence comprise a thermometer code. The position of the one-zero transition in this thermometer code indicates how far the start signal has propagated. This distance corresponds to the time interval $\Delta T$ which needed to be measured. The number of stages with an output HIGH value can be then calculated as:

$$
N=\frac{\Delta T}{T_{L S B}}
$$

where $T_{L S B}$ is the delay of a single delay element in the delay-line, which is equivalent to the resolution of this basic delay-line based TDC.

There is an issue with this architecture that should be mentioned. At any given point in time, a delay cell has either been activated by the start signal or it has not. 
Any intermediate state is not possible [8]. This results in nonlinearity, namely the quantization error $\varepsilon$, which can be described mathematically as follows:

$$
\Delta T=N \cdot T_{L S B}+\varepsilon
$$

Another design challenge is the stop clock signal distribution for the sample elements. We can see that if we design a TDC with this architecture requiring a large dynamic range (the capability to measure a relatively long time interval), an extremely long delay-chain is required. For example, with the commonly used IBM 0.13um CMOS technology, a thousand cells (e.g. inverters) delay chain would only be able to achieve a 16 ns dynamic range (assuming a standard cell propagation delay of $16 \mathrm{ps}$ ). Unfortunately, even with a thousand delay cells, a 16 ns dynamic range is still not sufficient for a practical TDC implementation. In additional, long chains have another problem. The sample elements need to be trigged simultaneously. Without any complementary methodology it becomes impossible to distribute the clock signal to all sample elements at the same point in time. The latency brought on by the long clock distribution network is very likely to cause a sample error in the final output thermometer code. One way to partially solve this problem is with a "clock tree" or "clock mesh" [12] [13]. For our basic delay-line based TDC, a tree structure clock network may be suitable to deliver the stop signals simultaneously.

The TDCs discussed so far are referred to as linear TDCs as they consist of one feed-forward delay-line without any feed-back. The length of the delay-line and therefore the area of the TDC grow with the maximum time interval to be measured. This can be avoided by the architecture that will be presented in next section. 


\subsection{Looped Time-to-digital Converter}

For long time interval measurements, an improved delay-line based technique has been developed, which is called the looped time-to-digital converter. This architecture uses a delay line feeding back into itself through a multiplexer. This feedback structure avoids increasing the number of delay elements in order to accommodate a long measurement interval (i.e. a large dynamic range). An example Looped TDC architecture is shown in Figure 2.7 [2, sec.4.2].

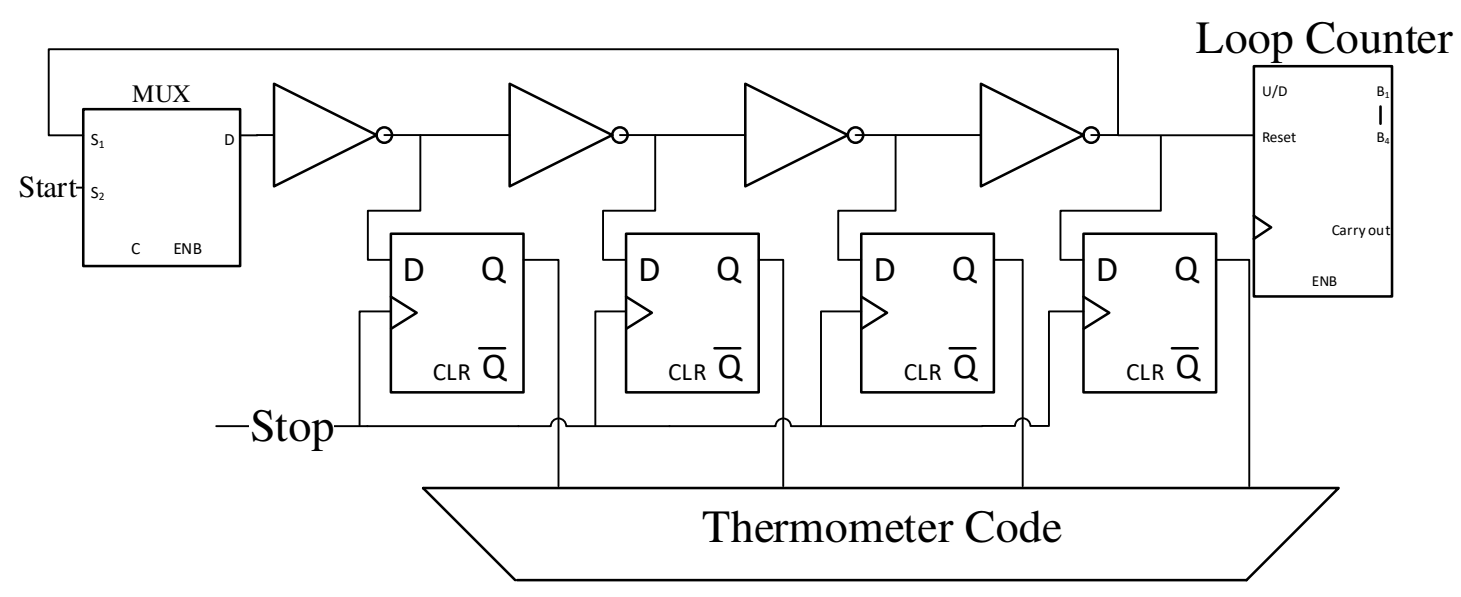

Fig. 2.7: An example of a looped time-to-digital converter architecture

The operating principle is illustrated as follows. The start signal propagates through the delay line (a inverter formed ring oscillator) and each loop traversal increments a loop counter. The binary code Bcnt, generated by the loop counter, directly provides a coarse quantization of the measured time. As with the basic TDC discussed above, a thermometer code is generated by an array of flip-flops which samples the outputs of the delay cells in the delay-loop. The one-zero transition of the thermometer code represents the signal stop position within one loop period. The fine resolution $\tau$ corresponds to the delay of each delay-cell in the loop. The overall time measurement value $\mathrm{B}$ can be calculated according to 


$$
B=N \cdot B+M \cdot \tau
$$

where $N$ is the overall count measured by the counter and $M$ is the number of delay elements within one cycle that the timing event has passed, as recorded by the thermometer code.

In principle a looped time-to-digital converter allows for arbitrarily long time intervals to be measured. Unfortunately a phenomenon known as "pulse shrinking" limits the maximum measurable time interval (Dynamic Range)[2, sec.5.4]. This happens because as the signal propagates through the delay chain its strength diminishes and it will eventually vanish. This parasitic pulse shrinking phenomenon shortens the circulating timing event. Additionally, the loop-closing element (e.g. a multiplexer as shown in Figure 2.7) will add delay asymmetry into the loop. This unbalanced delay accumulates for each loop cycle creating a measurement nonlinearity that cannot be ignored. 


\subsection{Gated Ring Oscillator Time-to-Digital Con- verter}

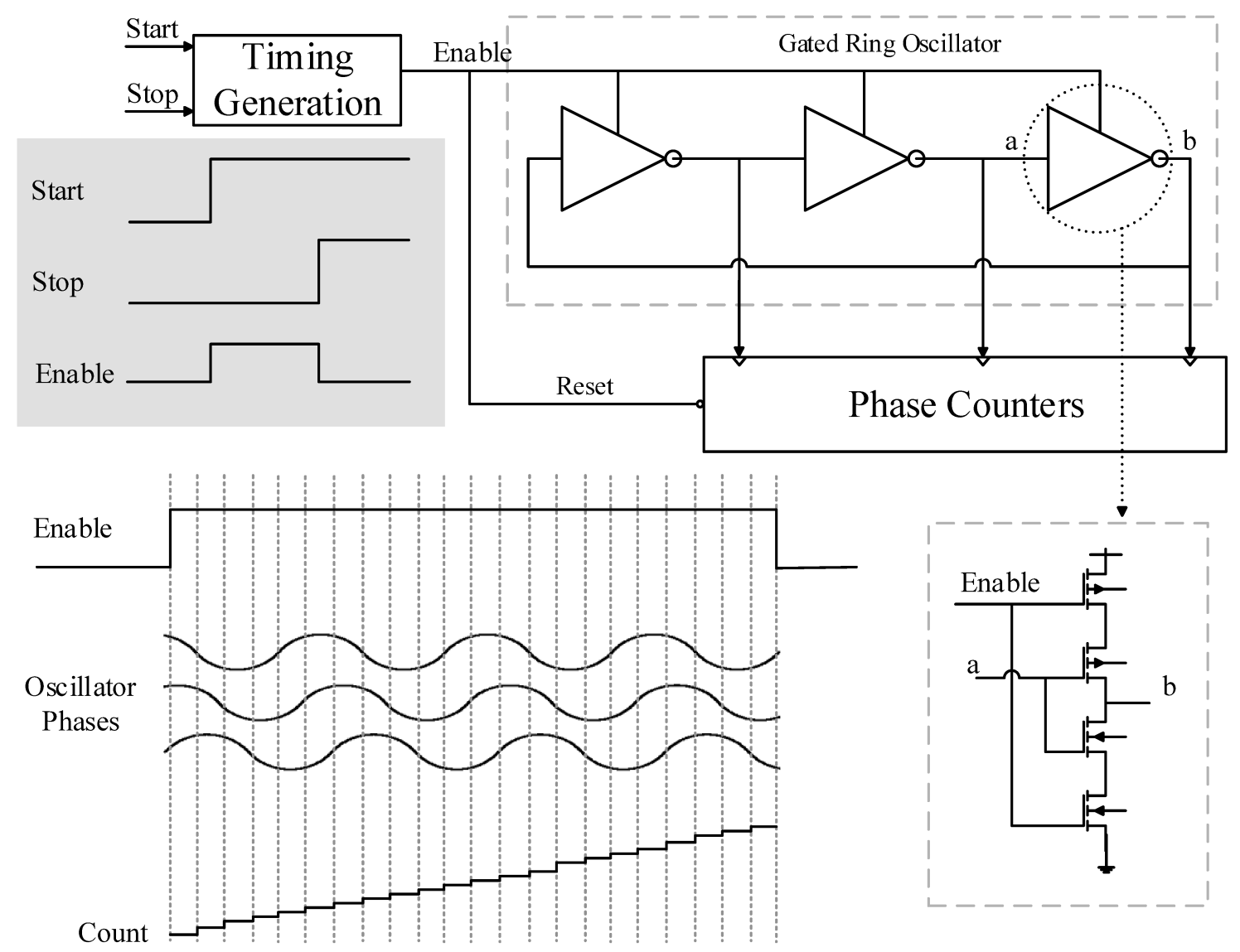

Fig. 2.8: A simplified circuit of GRO-TDC. A fundamental delay cell is implemented with a tri-state inverter.

The Gated Ring Oscillator(GRO) TDC is another architecture which is implemented with delay-line based loop configuration. As shown in Figure 2.8, this TDC architecture is similar to the Looped TDC which was introduced previously. However, there is a difference in the basic delay cell implementation between the two TDCs. Unlike a Looped TDC, the GRO TDC only allows the oscillator to have transitions during a given measurement. Therefore, the conventional inverter gate in a Looped 
TDC is replaced with a tri-state inverter in the GRO architecture. In addition, unlike the Looped TDC, the enable timing interval is created by a pre-generation block according to the time difference between the rising edges of start and stop signal. With this configuration, the delay asymmetry involved by the MUX stage in Looped TDC architecture is avoided.

Figure 2.8 also shows the operating mechanism of the GRO TDC. When the enable signal is high, oscillation occurs and the ring of inverters behaves identically to a typical ring oscillator. Conversely, when the enable signal is low, the inverter delay element is unable to charge or discharge the output capacitance, and as a result oscillation is suspended. During the measurement time interval, the signal propagated along the delay loop and skewed by each stage with a constant time delay $\left(T_{L S B}\right)$. A phase counter is used to detect each phase and forward the counting result to the next block which is normally a register.

Compare to the loop counter, this phase counter requires a short reaction time due to the fact that normally the ring oscillator is operating on a high frequency in gigahertz-level. Consequently, a high speed counter design challenge is involved by this block.

A GRO TDC normally consists of a relatively short delay loop. Therefore, the DNL of the step response is significantly reduced. In addition, GRT TDC has a good effective resolution by using intrinsic first-order noise shaping property [14]. These features make it suitable for being used in a Digital Phase-Locked-Loop or other similar systems. 


\subsection{Hierarchical Time-to-Digital Converter}

The TDCs discussed so far measure the complete time interval with the full resolution. A critical issue with this method is that its power consumption is very high, especially for higher resolutions. Also, the energy consumption grows linearly with the time interval length. For instance, consider the looped time-to-digital converter analyzed above. A long time interval can be measured with a relatively short delay line, but the gating signal may circulate in the delay loop for an unattractive high number of cycles, which linearly increases the required number of bits in the loop counter as well as the energy consumed in the counting process. Consequently, long time and high resolution measurements using the techniques discussed so far become unattractive.

One way to address this issue is to use a hierarchical TDC, as illustrated in Figure 2.9. The hierarchical TDC consists of two sub-TDCs, one of which has a fineresolution and the other a coarse resolution. In the coarse TDC, as in the looped TDC, timing calculations are achieved through the use of counters. In the fine-resolution TDC a small timing residue between a reference clock and the start signal is fed to a high resolution delay line [15]. While the fine-resolution TDC has a much higher resolution than the coarse $\mathrm{TDC}$, it has a small dynamic range, which in this case is equal to the quantization interval of the coarse TDC [8].

The coarse and fine resolution sub-TDCs are coupled by a multiplexer (Mux) and control logic. This multiplexer is the reason the theoretically simple and advantageous hierarchical TDC architecture is very challenging to implement. From the perspective of propagation delay, a multiplexer is not a symmetrical device with respect to its inputs, even if the topology is completely symmetrical (for instance by using a wiredNOR structure) [16]. Because of this, the layout and the wiring between the first TDC stage and the coupling MUX unavoidably introduce some asymmetry. The wiring is 


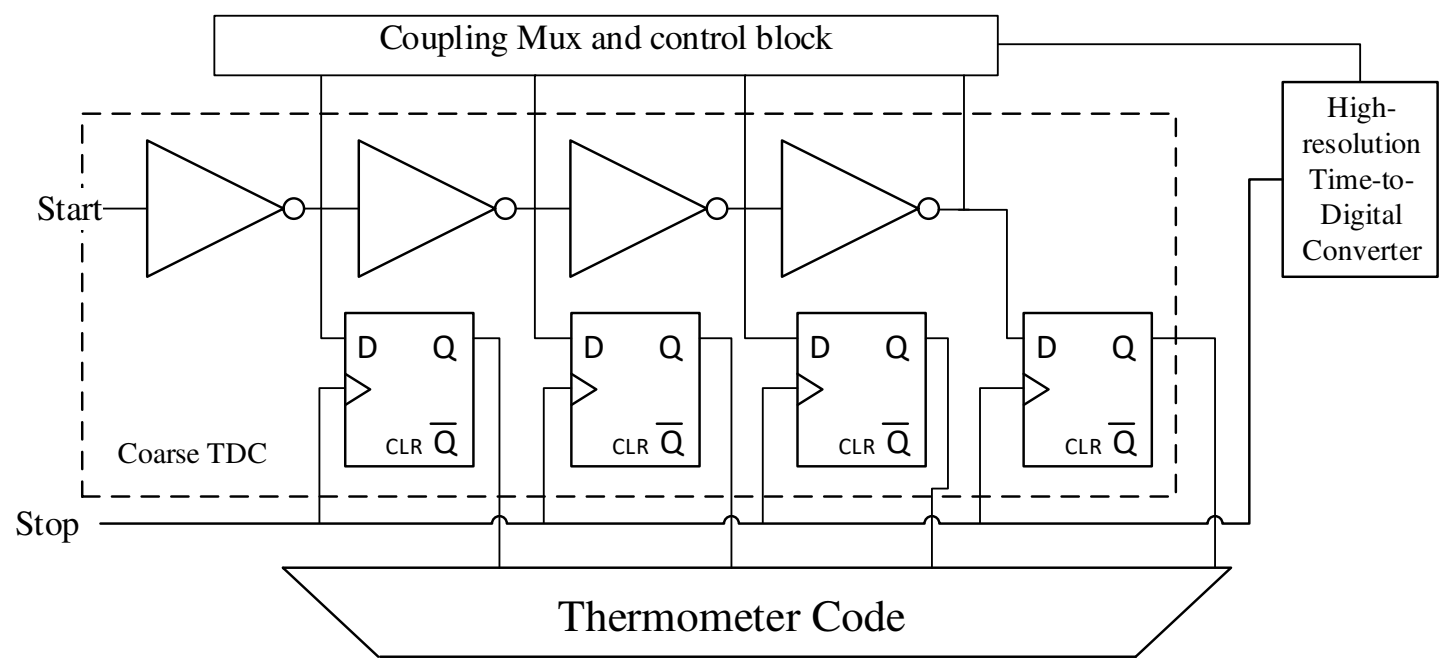

Fig. 2.9: An hierarchical time-to-digital converter architecture

particularly difficult as each input signal originates from another delay element. This causes a systematic nonlinearity in the hierarchical TDC measurement. Consequently, it makes the hierarchical approach unattractive for practical applications. For the injection of the residue time interval into the fine TDC the one-zero transition in the coarse TDC has to be detected first. This causes not only control logic overhead but also introduces some latency that has to be compensated by additional delay elements in the stop path between the coarse and fine TDC stages. Additional delay elements lead to additional power, area, and noise. Hence, the overall latency (i.e. the number of compensation elements) should be kept as small as possible.

Because of these issues, the coupling paths between the two TDCs has to be reduced as much as possible in order to take advantage of the hierarchical TDC architecture. An improved hierarchical architecture with a very short delay-line based first stage is presented in Figure 2.10.

The coarse TDC stage consists of a very short delay line formed with four coarse delay elements, which is coupled to the second stage at a single location. Similar to the 


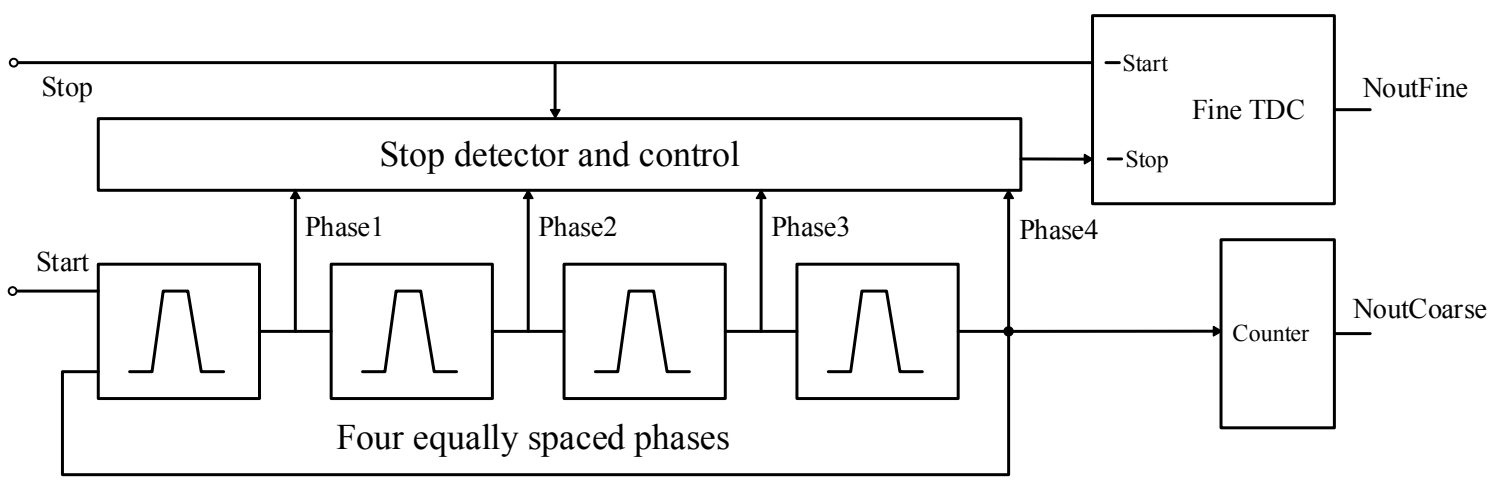

Fig. 2.10: An improved hierarchical time-to-digital converter architecture with a very short delay-line formed first stage

looped-TDC discussed in the last section, the short delay-line is bent over to achieve a long dynamic range. Each phase is connected to the fine TDC via a 4:1 multiplexer. The number of delay elements (and therefore the the fan-in of the multiplexer) is small enough to enable symmetrical layout. Similar to the basic hierarchical TDC discussed above, the measurement result is comprised of the output of the fine TDC NoutFine and the output of the coarse TDC NoutCoarse.

An example signal diagram is depicted in Figure 2.11. The circulating start timing event is injected into the fine TDC periodically. On the arrival of the stop signal both the coarse and fine TDCs are sampled. Then the output word of this hierarchical TDC is calculated according to $[2$, sec. 4.5$]$

$$
B_{\text {out }}=B_{\text {cnt }}+\frac{1}{4} B_{\text {phase }}+\left(\frac{1}{4}+\frac{1}{4} \kappa B_{F T D C}\right)
$$

where $B_{\text {cnt }}$ is the value of the loop counter, $B_{\text {phase }}$ is the number of the phase that has actually stopped the fine TDC, $B_{F T D C}$ is the measurement result of the fine TDC and $\kappa$ is the internal calibration coefficient. Due to the relatively shorter delay-line, the linearity of this improved hierarchical TDC is very good [2, sec.4.5].

Compared with the looped TDC, the hierarchical TDC achieves a relatively higher 

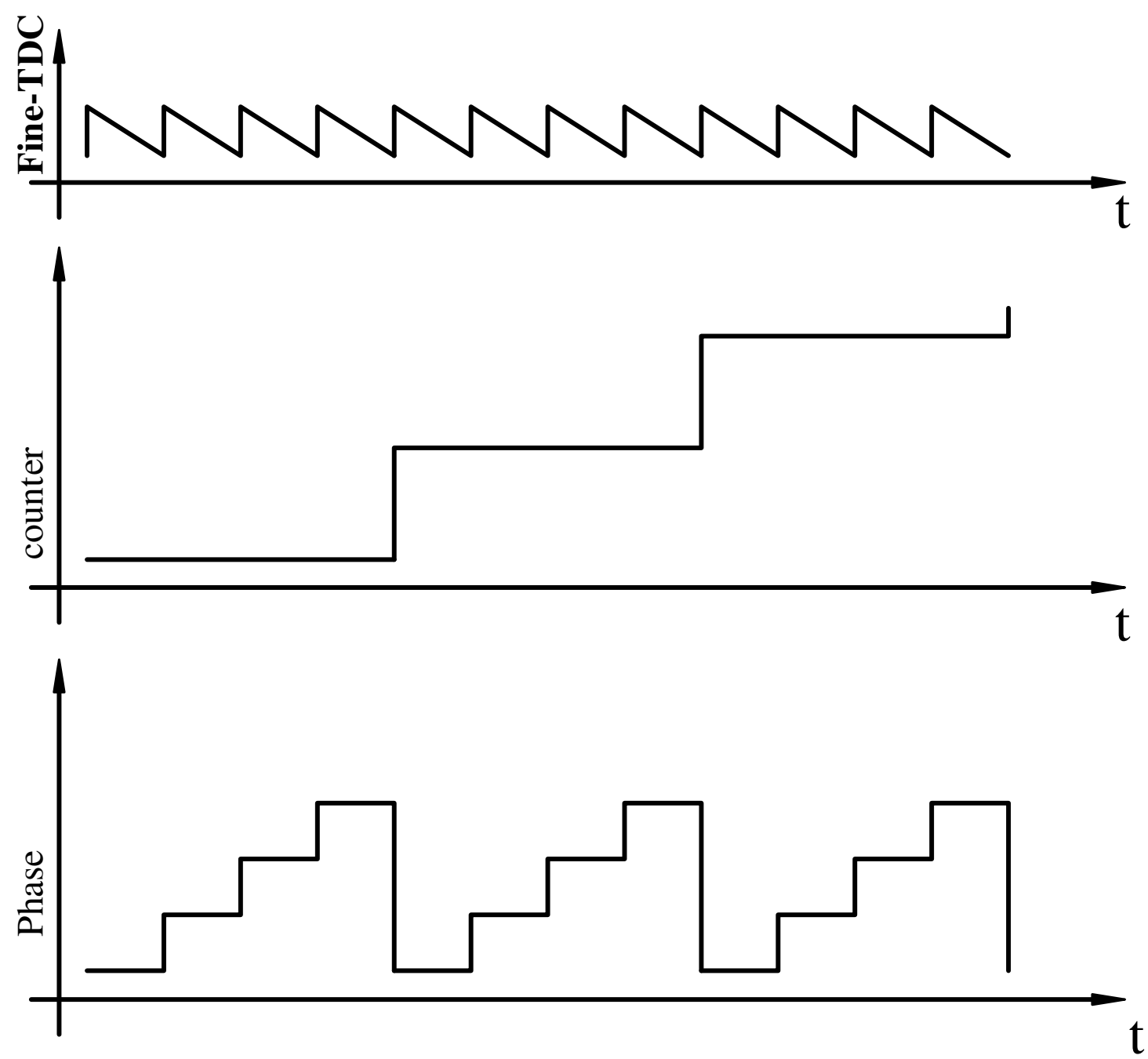

Fig. 2.11: Signal diagram of proposed hierarchical improved Time-to-Digital Converter

resolution and a similarly high dynamic range but with the cost of higher design complexity in order to reduce the systematic nonlinearity. 


\subsection{Vernier Time-to-Digital Converter}

The looped TDCs and hierarchical TDCs discussed above are the so called 2nd generation time-to-digital converters. As mentioned previously, the resolution of 2nd generation TDCs is limited by the propagation delay of one delay cell. In contrast, Vernier TDCs are capable of measuring time periods with a time resolution shorter than the fundamental delay through a single delay element [17]. TDCs that are capable of measuring time intervals with sub-gated delay resolution are usually referred to as 3rd generation TDCs [8]. As illustrated in Figure 2.12, this sub-gate-delay resolution of $T_{L S B}$ is achieved by using two delay chains with differing cell delays [18]: one for the start signal and one for the stop signal. The delay elements in the first delayline have a delay $t_{d 1}$ which is slightly larger than the the delay $t_{d 2}$ of the elements in the second chain.

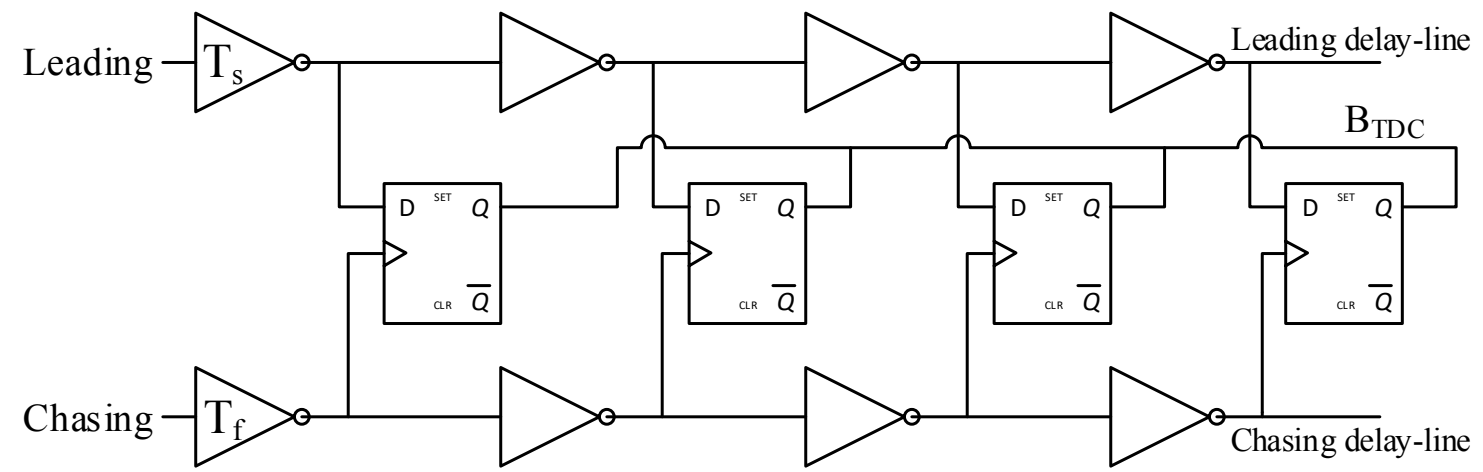

Fig. 2.12: Cut-out of a Vernier delay-line based TDC based on a first delay-line for the start signal and a second one for the stop signal. The arrival time at two corresponding nodes is assessed by early-late detectors such as flip-flops

During a measurement, the leading signal propagates along the first delay-line. The chasing signal occurs later, but the delays seen by this signal are smaller. Thus, the chasing signal catches up to the leading signal in the cascade of delay stages 
according to

$$
T_{L S B}=t_{d 1}-t_{d 2}
$$

The vernier TDC operating principle is depicted in Figure 2.13. The stage where both signals are in phase is detected by early-late detectors such as flip-flops, and its position is encoded in the one-zero transition in an output thermometer code. The position where this transition occurs represents the time/phase difference between the leading and chasing signal. With the Vernier technique, the resolution limitation can be effectively overcome but the penalty is in the extra area and power consumption required for the parallel delay chain. The skew between the signals in the leading and chasing delay line is reduced by $T_{L S B}$ in each stage for a certain dynamic range $T_{\text {total }}$. We can then determine that the number of stages required is given by [2, sec.5.3]

$$
N=\frac{T_{t o t a l}}{T_{L S B}}=\frac{T_{t o t a l}}{t_{d 1}-t_{d 2}}
$$

From Equation 2.7, we see that in order to attain a fine time resolution, the number of stages grows linearly with the required dynamic range $T_{\text {total }}$ and inversely with $T_{L S B}$. Hence, the Vernier TDC becomes impractically large when a large dynamic range is required. Fortunately, we can borrow some inspiration from the looped TDC discussed in Section 2.3: long measurement times can be achieved with reasonable area consumption by applying looped structures. In Vernier TDCs this is even more advisable as the length of the double delay-lines grows with the resolution. By taking the advantage of the loop architecture, the Vernier TDC is able to maintain a high resolution while having the capability of quantizing a relatively long time interval by using each delay element repeatedly. 


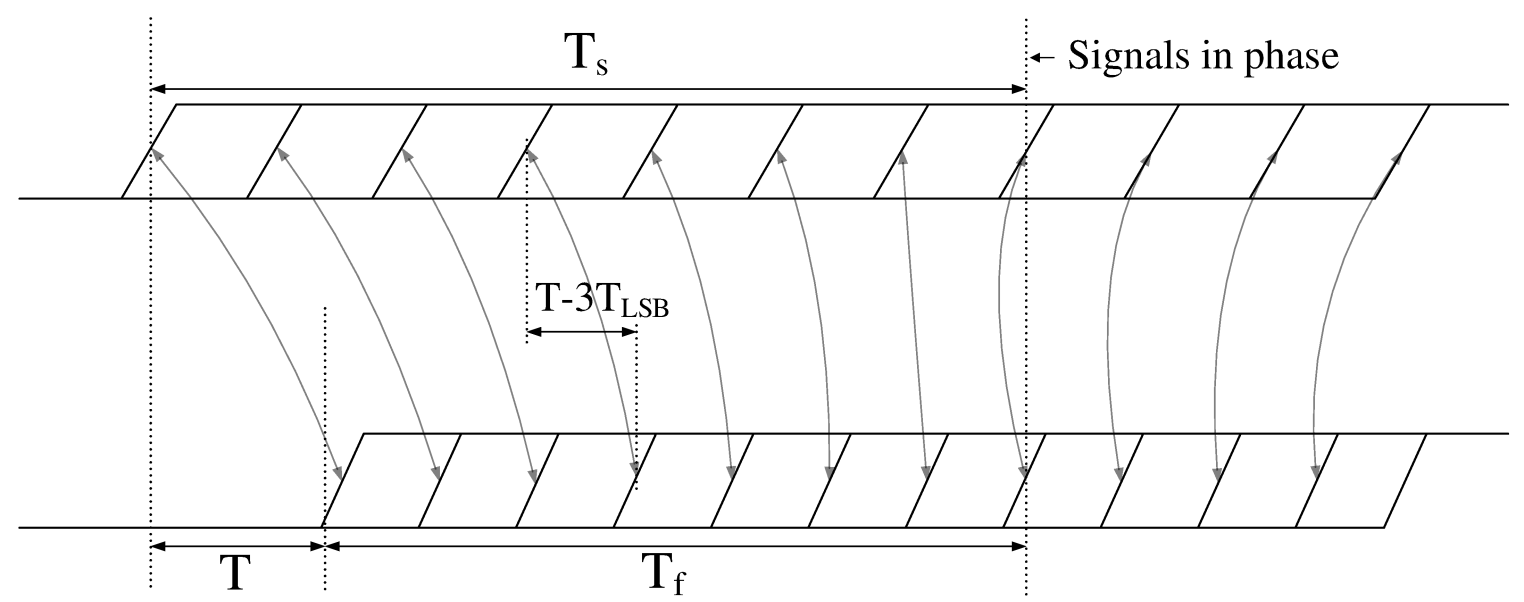

Fig. 2.13: Operating principle of Vernier delay-line based TDC. In each stage the chasing signal catches up the leading signal by one $T_{L S B}=t_{d 1}-t_{d 2}$

$[2$, sec.5.3]

\subsection{Conclusion}

Generally, for each of the previous TDCs mentioned, the achievable measurement resolution is inextricably tied to the performance of the delay cells. Ultimately, resolution improvement results from propagation delay optimization, implying delay cell switching speed optimization [19].

Table 2.1: Comparison of the state-of-the-art

\begin{tabular}{|l||c|c|c|}
\hline Reference & {$[14]$} & {$[20]$} & {$[21]$} \\
\hline Type & GRO & Hierarchical & Time Amplification \\
\hline Resolution (ps) & 6 & 476 & $0.98(\mathrm{FB}) / 6.01(\mathrm{FF})$ \\
\hline Dynamic range (bit) & 11 & 15 & $10(\mathrm{FB}) / 3(\mathrm{FF})$ \\
\hline Power Supply (V) & 1.5 & - & 1 \\
\hline Power $(\mathrm{mW})$ & $3.3-31.5$ & 6.3 & $3(\mathrm{FB}) / 17.5(\mathrm{FF})$ \\
\hline Technology $(\mathrm{nm})$ & 130 & 350 & 65 \\
\hline Area $\left(\mathrm{mm}^{2}\right)$ & 0.04 & 0.16 & 0.02 \\
\hline
\end{tabular}

Table. 2.1 provides a comparison of several TDC implementations. TDCs with a 
featured architecture may be superior on a certain parameter. A Time Amplification TDC normally achieves a relatively smaller resolution. However, it is hard for this architecture to achieve a relatively large DR. The reference [21] which is a Time Amplification TDC has two operation modes. The FB stands for the feedback mode and FF stands for the feedforward mode.

A hierarchical TDC saves the power consumption and the area consumption. However, the resolution of it is not desirable.

The GRO TDC in reference [14] was manufactured with the same CMOS technology of this work.

In the next chapter we will look at MOS-Current-Mode-Logic (MCML) gates family, which have properties that make it suitable for delay cell implementation and therefore resolution improvement. Furthermore, a novel TDC design based on an improved MCML-Buffer loop will be presented. 


\section{Chapter 3}

\section{System Level Design}

\subsection{Introduction}

The system level design of the TDC will be presented and analyzed in detail in this chapter. In addition, the advantages and disadvantages of MCML will be discussed and compared to conventional logic gates as well as their merits and demerits. As introduced before, a TDC acts as the interface between a time domain interval and a digitized signal. The basic block diagram is shown below in Figure 3.1. It converts a given length of time (in picoseconds or nanoseconds ) into its corresponding digital representation precisely.

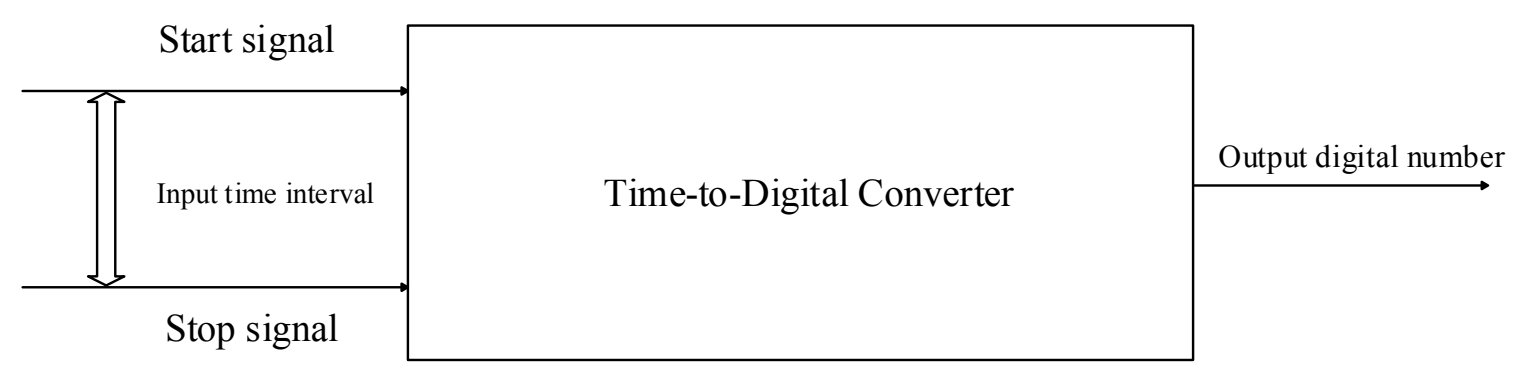

Fig. 3.1: Basic block diagram of a time-to-digital converter

First, a time difference between the start and stop signal is injected into the 
TDC. Then after a conversion process, the TDC outputs a digital number, which corresponds to this time interval. For this work, the output is a combination of binary codes and a thermometer code.

The following are the specifications that are considered the highest priority:

- Time domain resolution, $T_{\text {res }}$.

- Dynamic range, $D R$

- Power consumption, $P$

- Chip area, compared to the current state-of-the-art.

For all of the parameters above, the resolution is of the highest-priority. It defines the minimum time interval that can be measured and this is known as the signal shot precision (SSP) [22]. In order to improve the time domain resolution, most of the recent TDC research focuses on new TDC topologies. Comparatively little attention has been placed on the fundamental delay cell implementation. This work proposes an improved MOS-Current-Mode-Logic (MCML) based delay core. A TDC using this element maintains a relatively high resolution. Additionally, it has the ability to switch among four different resolutions, while scaling power consumption.

\subsection{TDC Module Diagram}

The TDC proposed here is depicted in Figure 3.2. It is comprised of a TDC core block and three peripheral blocks for counting, shifting and evaluating purposes.

The trigger event (start signal) is fed into the structure and then processed by the TDC core module. For the architecture using MCML gates, a pair of differential start signals is required. When the stop signal is activated, the measurement results are 


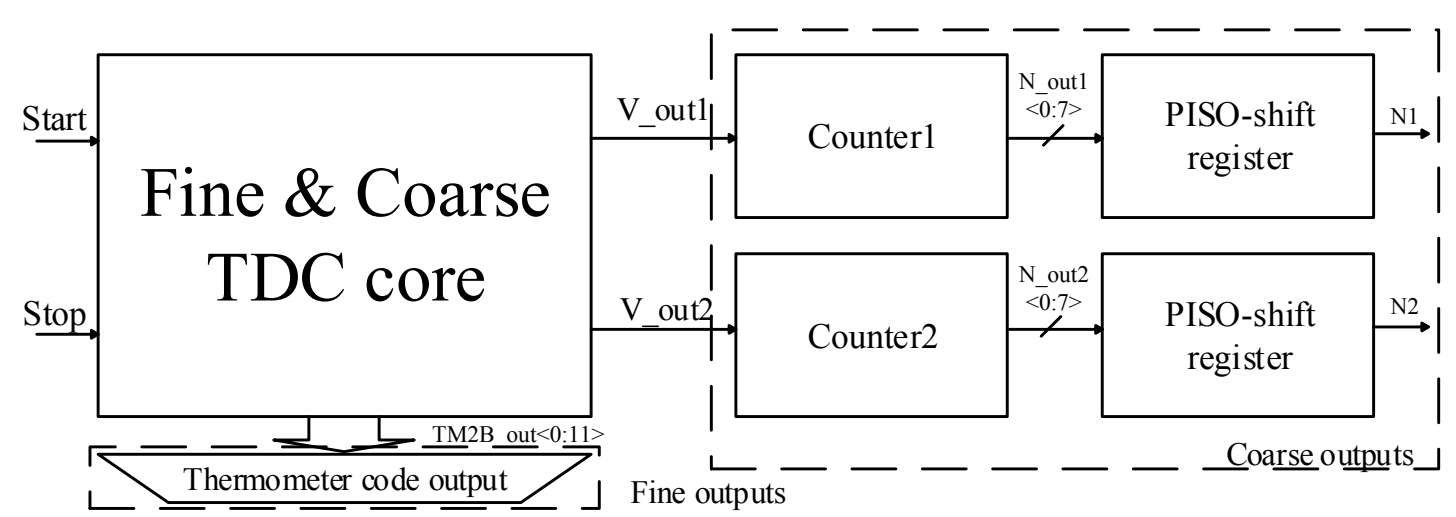

Fig. 3.2: TDC module diagram

given separately and shown in Figure 3.2. The coarse outputs $V_{\text {out } 1}$ and $V_{\text {out } 2}$, which are processed by a differential TDC core, are fed into two complementary counters, Counter 1 and Counter 2 individually. Next the parallel outputs of the counters $N_{\text {out } 1}$ and $N_{\text {out } 2}$ are converted to serial by a pair of shift registers used for parallel-in-serialout (PISO) conversion. On the other side, the Fine outputs, which result from a sample elements array inside the TDC core, are provided in a thermometer code. Each of the blocks will be discussed further in the rest of this chapter.

\subsection{Fundamental Delay Cell Design}

The trend in the TOF measurement field is developing circuits that can achieve high resolution while minimizing system complexity. Moreover, the Ultra-Deep-Submicron CMOS (UDSC) technologies provide high-performance standard logic cells [23]. With the benefits they bring, the resolution of TDCs (i.e. the propagation delay of each cell) can be improved. However, the cost of fabrication also increases as CMOS technologies are scaled down. For the purpose of reducing the cost without sacrificing the performance or increasing the power consumption or die area, a favorable candidate for high-resolution TDC design that has the ability to provide a smaller propagation 
delay than standard cells with the same CMOS technology is MCML. A exemplary MCML gate will be discussed in the next subsection.

\subsubsection{Basic One-level-MCML Circuit}

MCML logic is an attractive alternative to standard CMOS logic for delay chain implementation. An MCML gate consists of three main components: the pull-up resistance, differential pair, and a current sink. Figure 3.3 illustrates an example of a basic MCML delay cell.

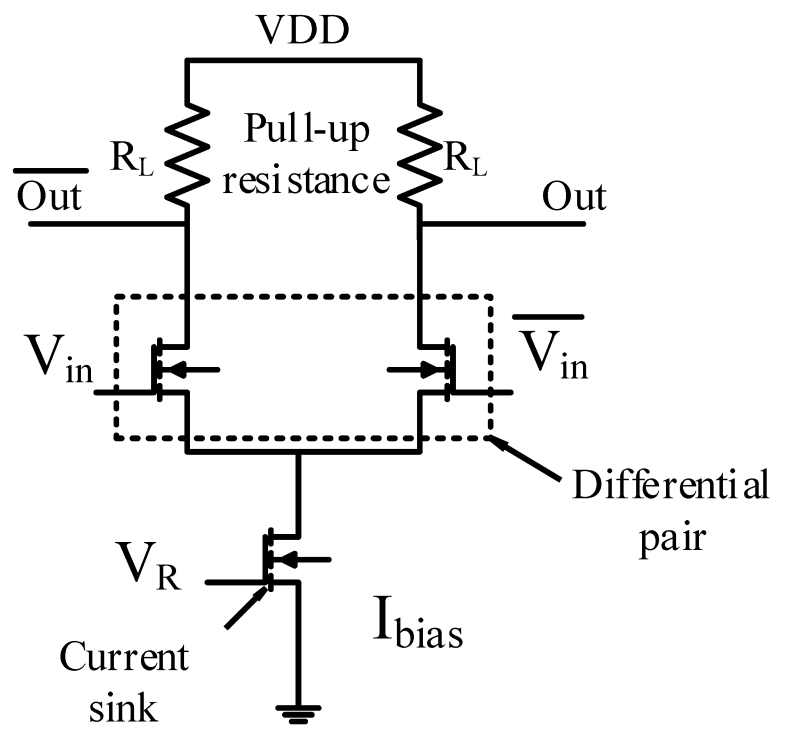

(a)

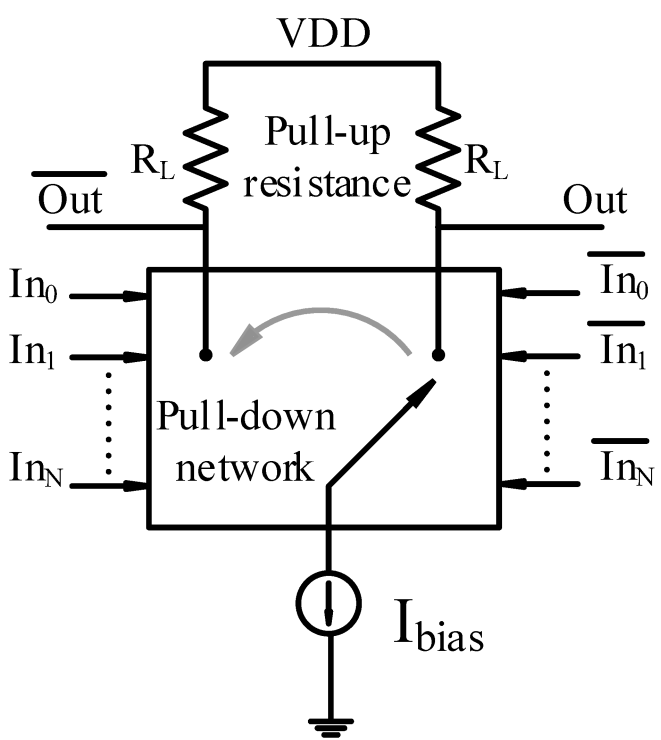

(b)

Fig. 3.3: An example of basic MCML circuits. (a)basic MCML inverter/buffer gate. (b)Current steering mechanism of the basic MCML gate

The pull-down network steers the bias current, $I_{s s}$ between the load devices. The loads $R_{L}$ can be either active devices or passive resistors. In the case of the resistive loads shown in Figure 3.3, the voltage at the output nodes varies between $V_{D D}$ and $V_{D D^{-}} I_{s s} R_{L}$ as the bias current is selectively steered through one load or the other [24]. Compared with the conventional CMOS logic gate, MCML circuits can operate faster 
since propagation delay can be reduced by reducing the voltage swing range [25].

\subsubsection{Design Parameters}

A. Delay $\left(t_{d}\right)$

The small-signal model for the inverter/buffer in Figure 3.3, can be approximated by the equivalent RC circuit in Figure 3.4 [24]. The small-signal approximation is used because MCML gates experience a small input voltage swing. $C_{L}$ is the load capacitance and is given by [26]

$$
C_{L}=C_{\text {wiring }}+n C_{i n}=C_{\text {wiring }}+n \times \frac{2}{3} C_{o x} W_{n} L_{n}
$$

where $C_{\text {wiring }}$ is the wiring capacitance and $n$ is the gate fan-out. Using first-order circuit analysis [27], the gate delay $t_{d}$ is given by [26]

$$
t_{d}=0.69 R C=0.69 R\left(C_{d b, n}+C_{g d, n}+C_{L}\right)
$$

where the $C_{g d}, C_{d b}$, and $C_{i n}$ are the gate-to-drain overlap, drain diffusion, and input capacitances of the differential pair NMOS respectively. The $C$ is the total capacitance of $C_{g d}, C_{d b}$, and $C_{i n}$. The $R$ is the pull-up loads of the RC circuit in Figure 3.4.

For the purpose of high-resolution TDC design, the propagation delay is the most important parameter. It will be treated carefully for the rest of this thesis.

B. Current $(I)$ and Power Dissipation $\left(P_{d}\right)$

A critical feature of MCML gates is that they drain a constant current $I$ and do not dissipate dynamic power. This is because when one of the differential outputs is charging $C_{l}$, the other one is discharging an equal capacitance. As a result, only 

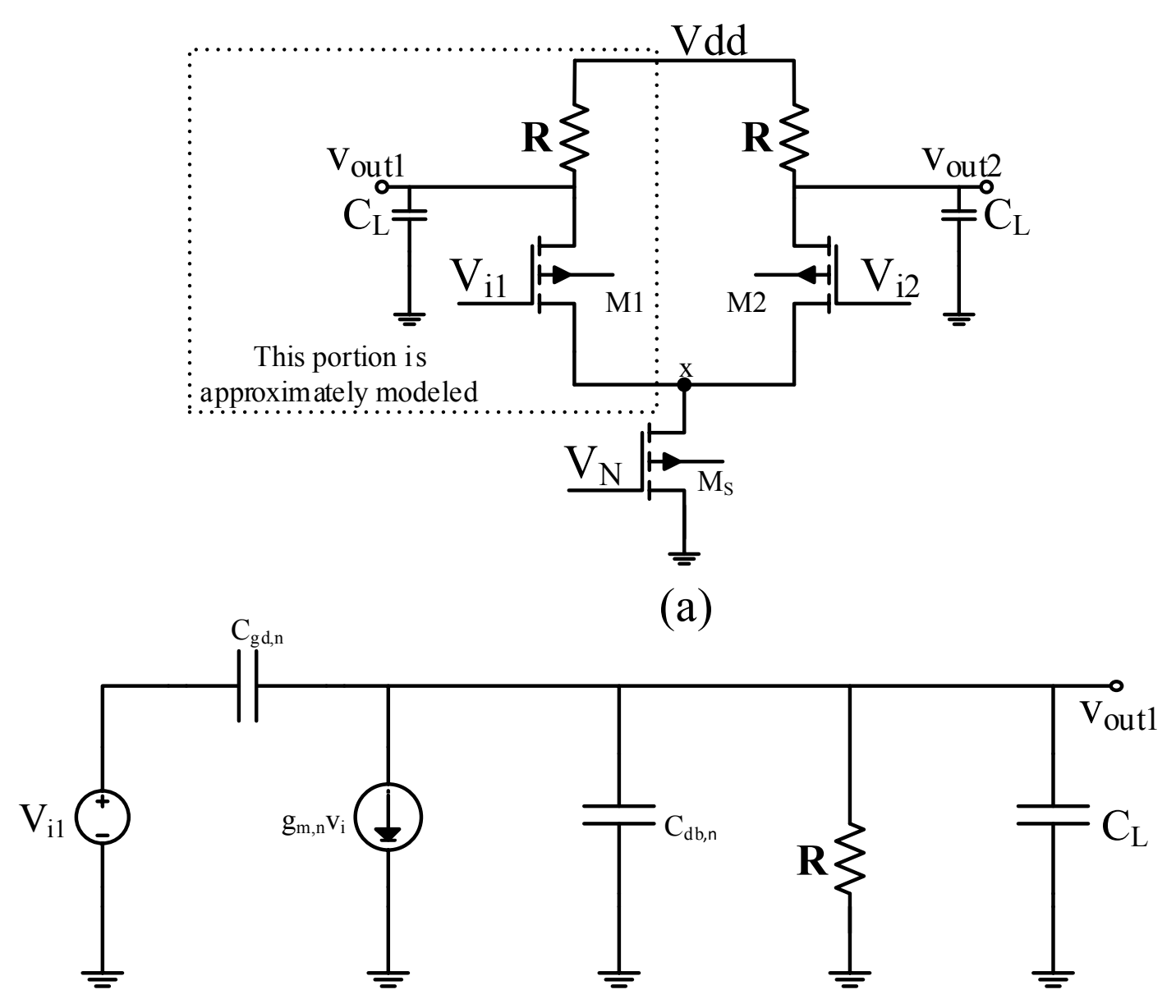

(b)

Fig. 3.4: MCML delay cell analysis. (a) A one-level MCML gate schematic. The highlight portion is approximately modeled in the sub figure below (b) Equivalent small signal model of the hight light portion

static power is considered:

$$
P_{d}=V_{D D} \times I_{\text {bias }}
$$

The $I_{\text {bias }}$ is the saturation current of the bottom NFET in Figure 3.4 and is expressed by

$$
I_{b i a s}=W_{s} v_{s a t} C_{o x}\left(V_{n}-V_{t h}-A_{b u l k} V_{d s a t}\right)\left(1+\frac{V_{x}-V_{d s a t}}{V_{A}}\right)
$$

where $W_{s}$ is the width of the NFET $M_{s}$ and $v_{s a t}$ is the saturation velocity, $A_{b u l k}$ is 
the bulk charge effect parameter, $V_{t h}$ is the threshold voltage, and $V_{A}$ is the early voltage of the bottom NFET $W_{s}$. $V_{d s a t}$ is the saturation drain voltage of $M_{s}$. $V_{x}$ is the voltage at the internal point $x$ which is shown in Figure 3.4 (a) [28].

It should be mentioned that MCML gates do not always consume less power than conventional gates. The power of standard CMOS logic gates increase with the operation frequency and the MCML has a constant power consumption. This means that the power of standard CMOS gates will becomes higher than the power of MCML above a certain frequency (approximately $300 \mathrm{MHz}$ ). This makes the MCML a good candidate at high speed circuit design.

\section{Voltage swing $(\Delta V)$}

The bottom NFET in Figure 3.3 (a) generates a constant current, $I_{\text {bias }}$, which is steered to one of the two branches depending on the inputs. For example, the left branch results in the low output $V_{D D}-\Delta V$, while the right branch results in the high output $V_{D D}$, where the voltage swing or voltage drop $\Delta V$ across the load resistance is equal to

$$
\Delta V=I_{\text {bias }} \times R_{L}
$$

According to Equation 3.5, the upper bound of $\Delta V$ is set by the saturation region of the bottom NFET.

Compared to a full-swing device, the MCML gate achieves a smaller propagation delay with the price of smaller voltage swing. Thus, this logic family becomes attractive when implementing the TDC delay chain with MCML gates as its delay cell. In addition, the differential nature of MCML enhances the common mode noise rejection of the circuit [26]. As well, the power consumption of the MCML delay loop can be less than that of a conventional CMOS inverter chain above a certain loop frequency, since conventional logic power requirements scale in proportion to frequency while 
MCML power requirements are constant.

Finally, the MCML-based TDC achieves not only a smaller propagation delay compared to the conventional logic gates, but also a lower circuitry complexity by taking advantage of its differential output for inversion. Moreover, a single MCML gate can achieve either a buffer or an inverter gate by its differential outputs. These features are presented in the next subsection, where an improved switchable MCML delay cell is presented. It serves as the basic delay element of the TDC design.

\subsubsection{An Improved Switchable MCML Delay Cell}

Recent TDC research focuses on new TDC topologies, such as looped Vernier TDCs and local passive interpolation TDCs [29]. Comparatively little focus has been placed on the fundamental delay cell implementation. This work proposes a 4-step selectable delay MCML-based delay cell as shown in Figure 3.5. The cell delay is inherently adjustable due to the inclusion of the four selectively switched active PMOS loads, which in practice influence the voltage swing at the output of the cell. Consequently, a TDC utilizing this element as its fundamental delay cell can operate at different resolutions.

The four switching steps are controlled using reference voltages $V_{p 0}$ to $V_{p 3}$.

\subsection{MCML-based TDC Core Design and Improve- ment}

As the most important block in the TDC system, the TDC core serves as the signal processing unit. It takes the injected start and stop events and converts the corresponding time difference into its output digital representation. Among all of the 


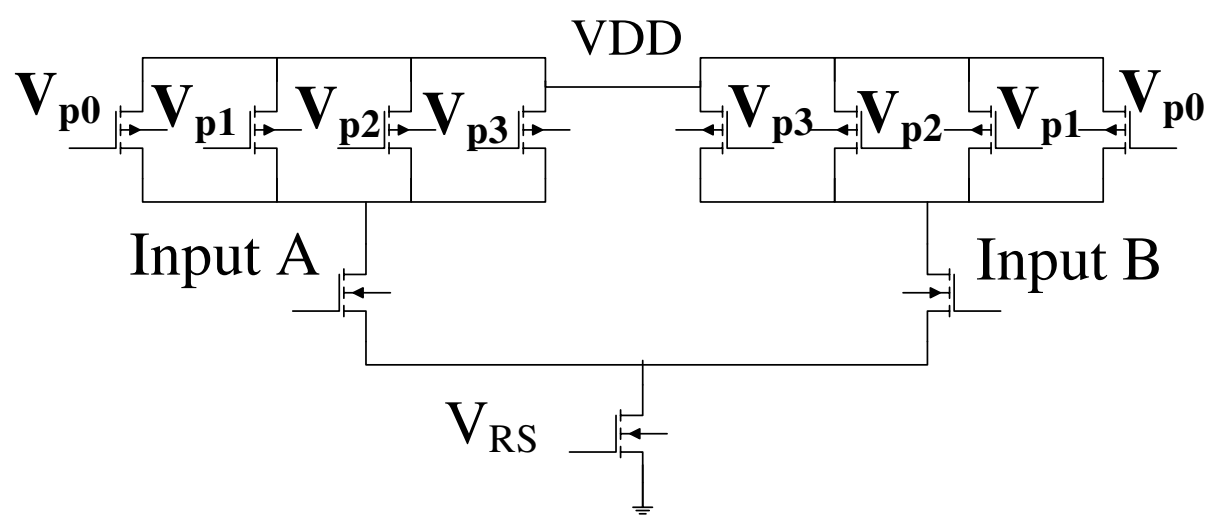

Fig. 3.5: Diagram of steps switchable MCML-buffer

modules introduced previously, the design of TDC core block is the most challenging. In this work, the TDC core is implemented using the switchable MCML gates discussed previously as its delay cells.

\subsubsection{Basic MCML-buffer-based TDC Core}

As discussed in the previous section MCML gates may be used as delay cells for high resolution and low power TDC design. A primary TDC core that uses this fast operational MCML gate is designed. The MCML TDC consists of a differential MCML buffer delay chain and two sample element (flip-flops in this case) arrays. As shown in Figure 3.6, the main delay chain is formed by using $n$ stages of switchable MCML buffers. At the beginning of measurement, the start event propagates along the delay chain and is skewed by each stage by a constant propagation delay $T_{L S B}$. Consequently, the maximum time interval can be measured by this TDC is $n \times T_{L S B}$. The start signal passes each MCML gate only once. Assuming the stop signal occurs before the start signal reaches the end of the chain, the number of cells that the start event has traversed represents the quantization result. This result is quantized by all sample elements loaded by each delay cell. Due to the differential nature of MCML 
stages, each stage is sampled by a pair of flip-flops (FFs). Next an n-bit thermometer code is obtained by the sample array. The measured result is calculated with the number of logic ones in the quantized thermometer code.

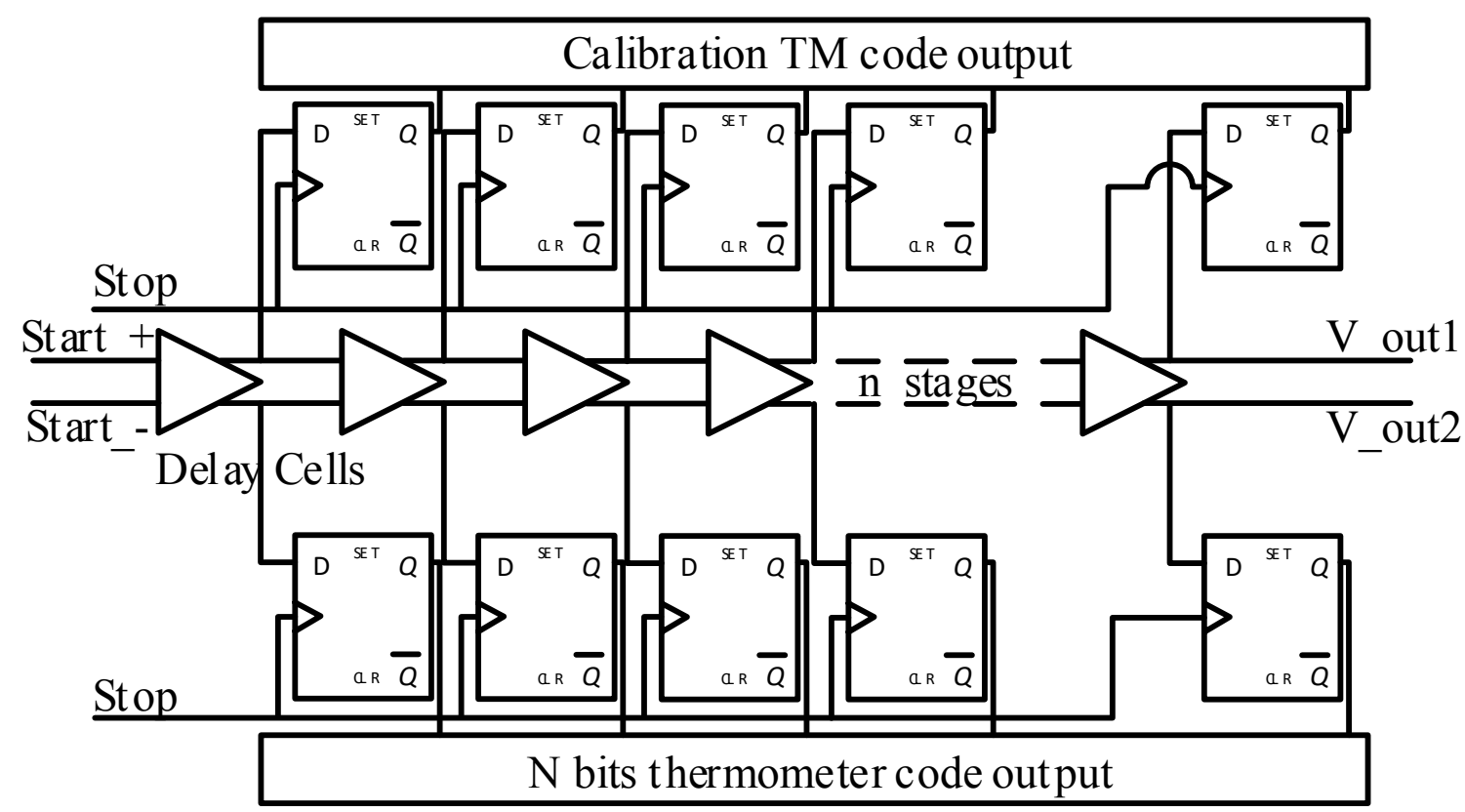

Fig. 3.6: Diagram of the basic MCML-Buffer based TDC core schematic

The signal diagram is shown in Figure 3.7. As illustrated previously, the start signal is skewed by each delay cell with a constant delay $T_{L S B}$. Hence, the Start $_{01}$ is the delayed version of start signal skewed with one $T_{L S B}$. Start $t_{02}$ is the delayed version of start signal skewed with two $T_{L S B}$, and so forth. Assuming the stop signal happens $x \times T_{L S B}$ after the start event (differential signal in this case), all the FFs are triggered at its rising edge. In this point of time, $x$ stages has been passed by the start signal. Therefore, a thermometer code that contains $x$ bits of logic 1 and $n-x$ bits of logic 0 is acquired. The location of the one-zero transition in the thermometer tells the quantized result of the conversion, namely the digital representation of the time interval to be measured. 
For this architecture, an extremely large number of stages $n$ is required if the purpose is to design a TDC with a large dynamic range. (For example, a TDC is implemented using IBM $0.13 \mu \mathrm{m}$ technology. Assuming the propagation delay of each cell is 16 ps, a 160 ns DR would require 10000 stages). This leads to not only an increased area and power consumption but also a reduced time domain resolution. When a delay chain is relatively long, a phenomenon called pulse shrinking occurs [30] [31]. As the number of stages is increased, the Differential non-linearity (DNL) accumulates along the delay chain [32]. The start event that propagates through the delay chain will eventually vanish due to this phenomenon. Hence, using a long delay chain architecture to achieve a large dynamic range is impractical. For the purpose of overcoming this issue, an improved MCML-based TDC architecture with loop configuration is presented in the next subsection.

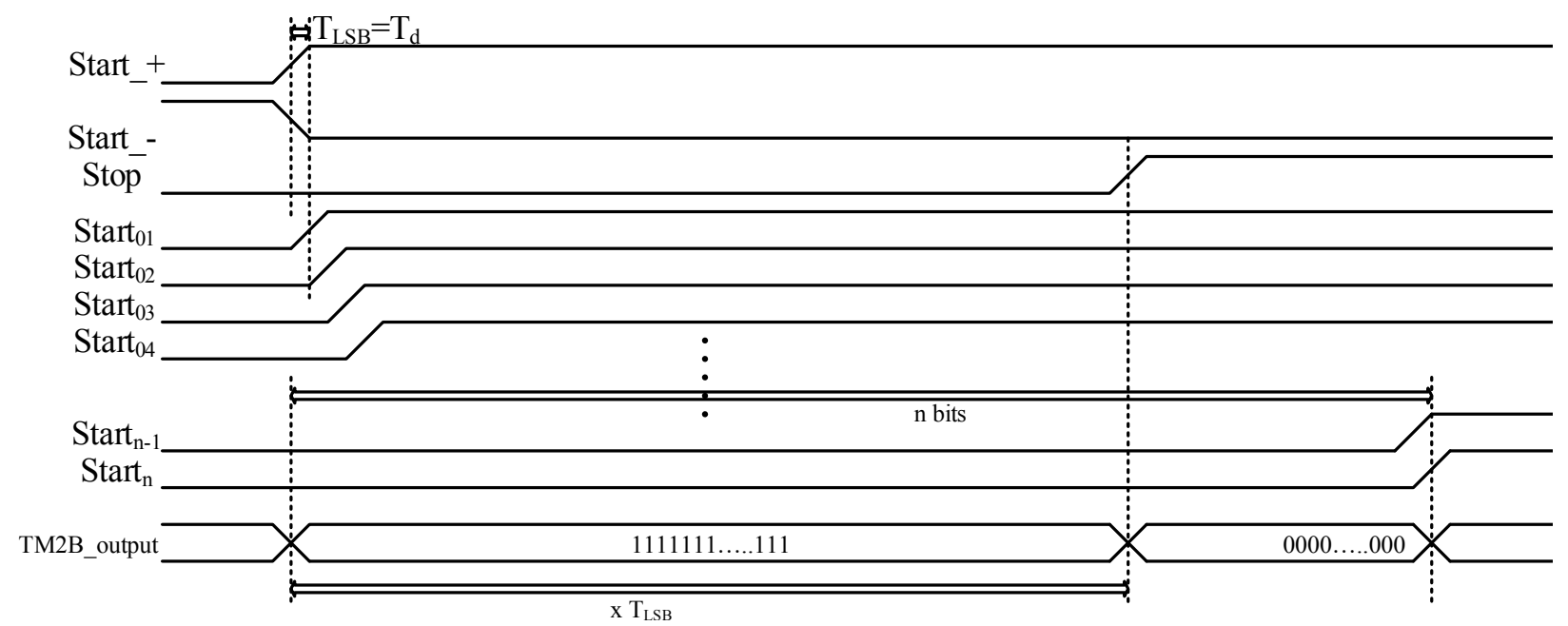

Fig. 3.7: Signal diagram of the TDC core schematic in Fig.3.6 


\subsubsection{An improved 4-steps Switchable MCML-based TDC core}

The TDC discussed above is a so-called linear TDC as it consists of a feed-forward delay line without any feedback. The length of this TDC core and the area of the TDC grow with the dynamic range. This can be avoided by using a looped MCMLbased TDC architecture where a short MCML-based delay line is bent into a loop and traversed several times by the start event. An improved 4 step switchable MCMLBuffer based TDC core is shown in Figure 3.8. A ring closer block is used to form the closed loop. It should be mentioned that this block has its own propagation delay and may introduce delay asymmetry into the delay loop. Hence, it was carefully designed and the propagation delay of this block is optimized to have the same delay of a MCML-buffer. 12 improved MCML-buffer stages are involved in the delay loop. $V_{\text {out } 1}$ and $V_{\text {out } 2}$ are the two differential outputs.

Two counters are used to count the number of times the start signal has circulated around the loop. Usually, a conventional CMOS looped TDC requires a dual-edgetriggered counter which involves extra design complexity. In this design, a singleedge-trigger achieves the required quantization range. The MCML based TDC core includes complementary delay paths, and the differential start signal operates in each of the delay paths with a $180^{\circ}$ phase difference. This differential characteristic is advantageous as a single-edge triggered counter is involved in the upper delay path and another compensating single-edge-triggered counter is connected into the lower delay path. Consequently, for the same number of loop counter bits this architecture achieves two times the quantization range, compared to a conventional looped-TDC configuration (using one loop counter to record a single delay line) .

An example signal diagram is depicted in Figure 3.9. When the start signals are 


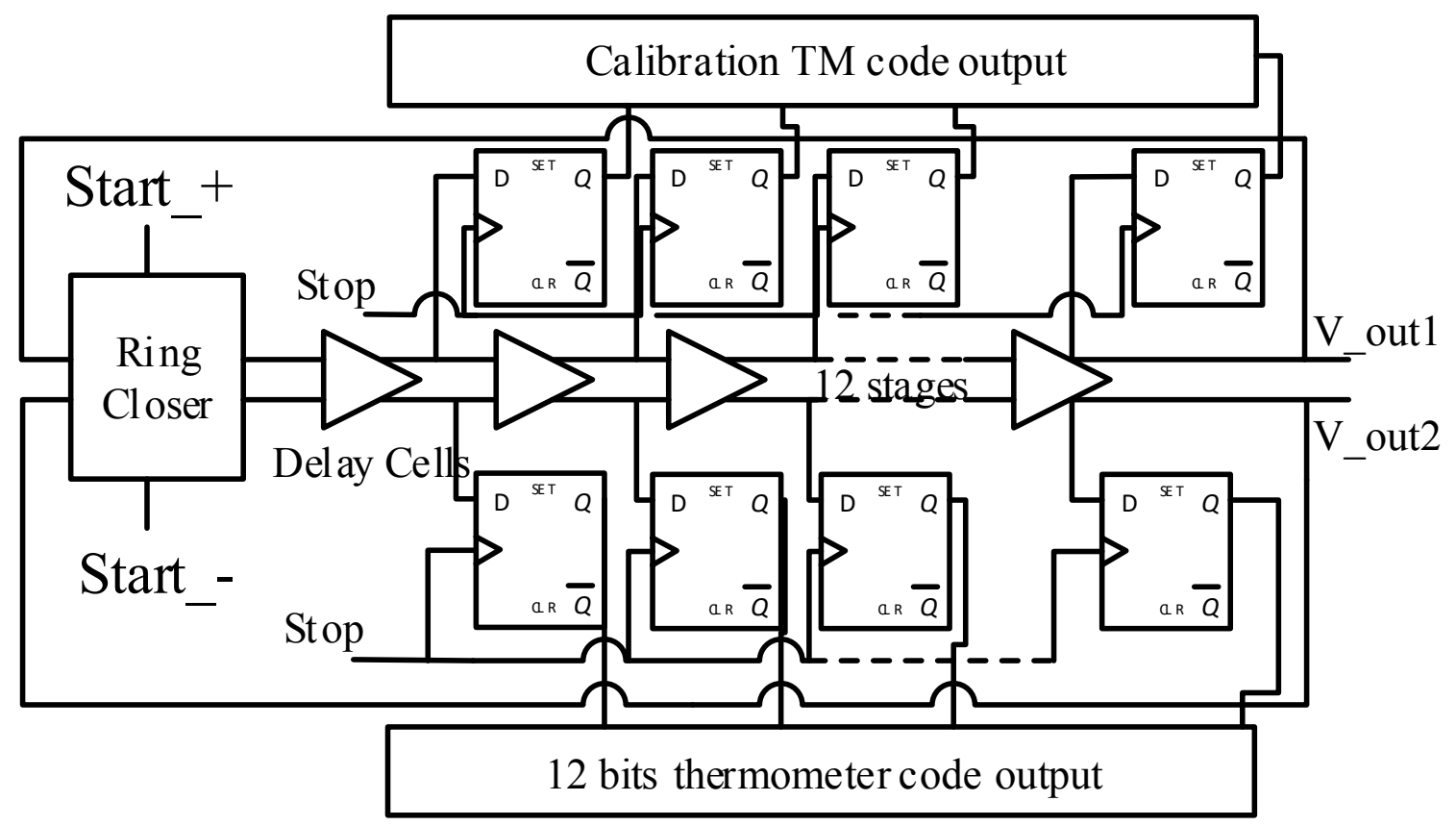

Fig. 3.8: Diagram of the improved 4 step switchable MCML-Buffer based TDC core

injected, both of the two complementary loops begin to oscillate. When the stop signal is received, both the flip-flop array and the counters are sampled with a simple clock distribution network. The loop counters output two parallel 8-bit quantized binary codes $N_{1}$ and $N_{2}$, corresponding to the number of cycles traversed in each loop. The sum of $N_{1}$ and $N_{2}$ is the total number of cycles, and the total time measurement result is given by

$$
T_{\text {total }}=N_{\text {res }} T_{L S B}+\left(N_{1}+N_{2}\right) T_{\text {loop }}
$$

where $N_{\text {res }}$ is the number of ones in the output thermometer code and $T_{\text {loop }}$ is the delay loop period.

The time residue within one loop period is loaded in parallel into a flip-flop array and the one-zero transition in the 12-bit thermometer code represents the residual quantization result. The lower path sample array also generates a complementary thermometer code which can be further used in a self-calibration mechanism. After 


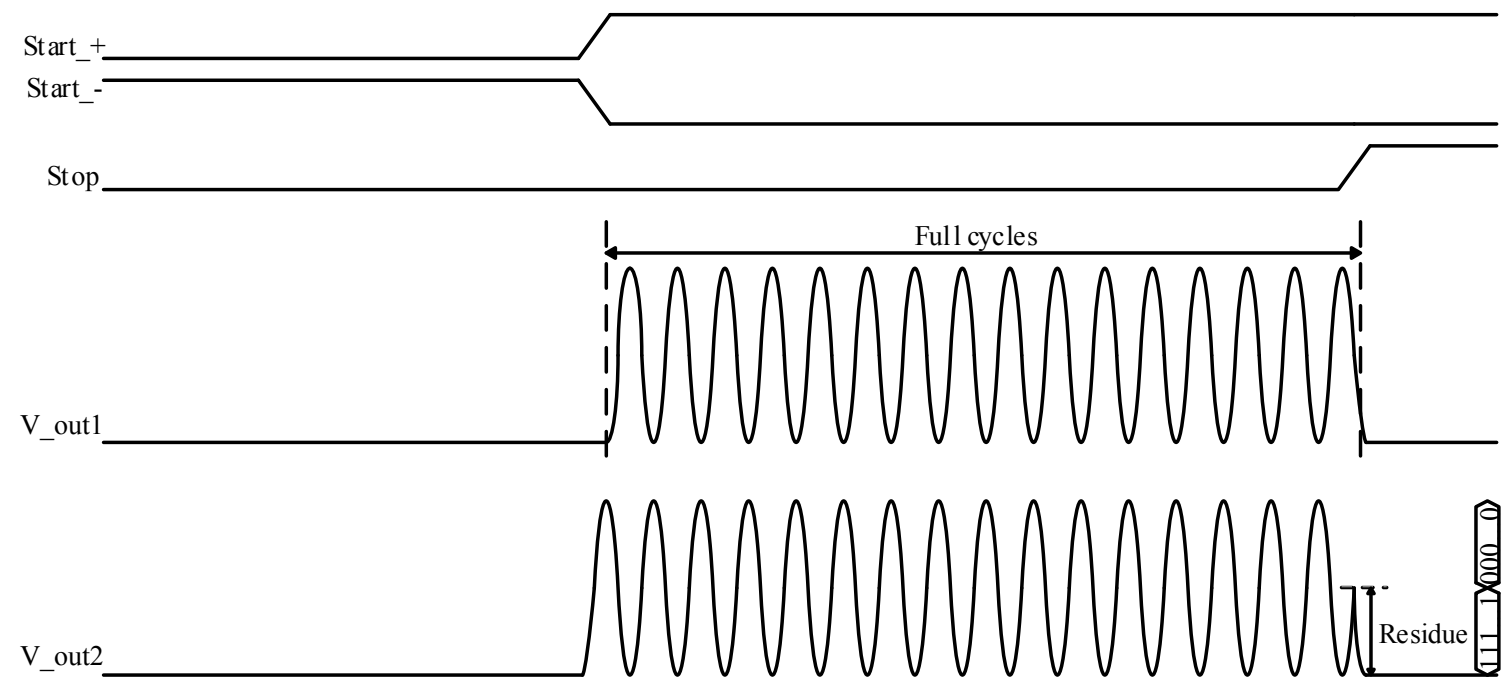

Fig. 3.9: An example signal of the switchable MCML-Buffer based TDC core

the stop event, the binary codes generated by the two counters are shifted into a serial output by parallel-in-serial-out shift registers. The calculation process is then carried out by a peripheral circuit.

A specific example is given in order to fully understand the operation. Assume that the $T_{L S B}$ is $16 p s$ and number of the delay cells $n$ is 20 . In addition, the stop event occurs 10 ns after start event. From the Equation 3.6, it can be calculated that the loop cycle $T_{\text {loop }}$ is $16 \times 20=320$ s. After the full time interval is divided by the loop cycle, 31 full cycles is acquired with a residue of $0.25 T_{\text {loop }}$. This means that in the last cycle, the start event is sampled at the 5 th stage $\left(N_{\text {res }}=5\right)$ and failed to reach the end. Consequently, the output thermometer code should be 11111000000000000000 . From another aspect, the counting results $N_{1}$ and $N_{2}$ should be 16 and 15 separately.

For this example, the result ends up with two components. The counting results $N_{\text {out } 1}$ and $N_{\text {out } 2}$ of the two loop counters that corresponds to 16 and 15 separately. The converted time domain representation of the full cycles part is equal to $T_{\text {loop }} \times(16+15)$ $=9920$ ps. Then the thermometer code gives 5 logic HIGH outputs which is equal 
to $0.25 T_{\text {loop }}$. The sampled time domain representation of the residue part is equal to $0.25 \times 320 p s=80 \mathrm{ps}$. Ideally, the sum of these results is $10000 \mathrm{ps}$ which is matched to the reference time interval $(10 \mathrm{~ns})$.

\subsection{Peripheral Circuits}

In this work, a few peripheral building blocks are used to process the measurement results that are output by the TDC core. The ring closer closes the feed-forward delay line into a loop. The loop counters record the numbers of loop cycles that are traversed by the timing event. Finally, the Parallel-In-Serial-Out (PISO) shifter register is responsible for binary code conversion.

\subsubsection{Ring Closer}

In contrast with the conventional gates based Looped TDC, which used a 2-to-1 Multiplexer to form the closed delay loop [33], the MCML gates require a differential closing block due to their properties. Hence, a novel ring closer which is suited for the differential structure loop is designed. As shown in Figure 3.10, the ring closer is formed by two NAND gates. Using a cross-coupled connection, it passes both the external start event and the feedback signal to the first delay stage.

\subsubsection{Loop Counter}

The expression "loop cycle" $\left(T_{\text {loop }}\right)$ is used to describe time length of each full cycle. Therefore, a counter is required to determine how many times the delay line has been passed by the start event before the TDC is stopped. The loop counters discussed previously is designed by using a widely used circuit [34]. The 8-bit counter 


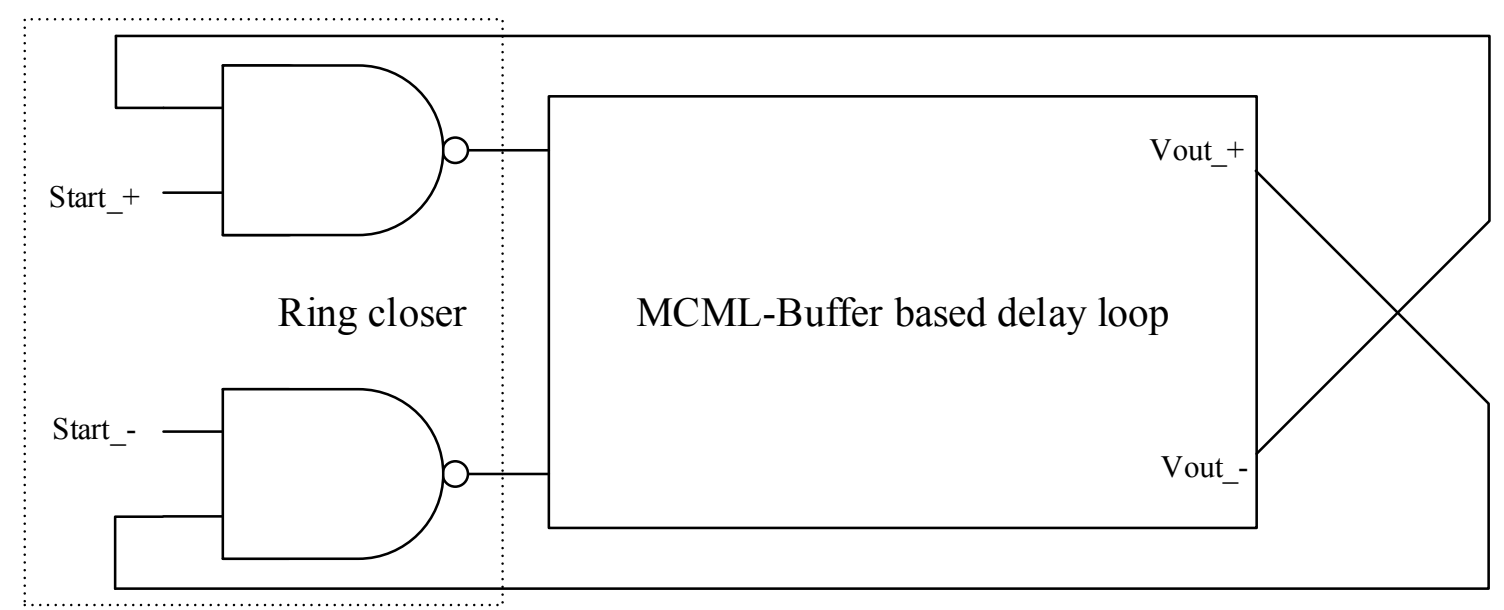

Fig. 3.10: Diagram of the Ring closer module formed by 2 cross coupled $2 x 1$ NAND gates

is composed of a FF chain and the logic correction elements (inverters in this case).

For the conventional looped TDC [8], the inverter based delay loop requires only one counter to record the state switching. However, that also means both the rising and falling edge of the signal represent a full cycle and need to be detected by the counter. The design of dual-edge triggered storage elements (DETSE) are challenging [35]. Fortunately, the MCML-based delay loop used in this work divides the propagation signal into two parts with a $180^{\circ}$ phase difference. Therefore, we can simply count the rising edges only by using a pair of basic counters. The circuit of a 8-bit counter is shown in Figure 3.11.

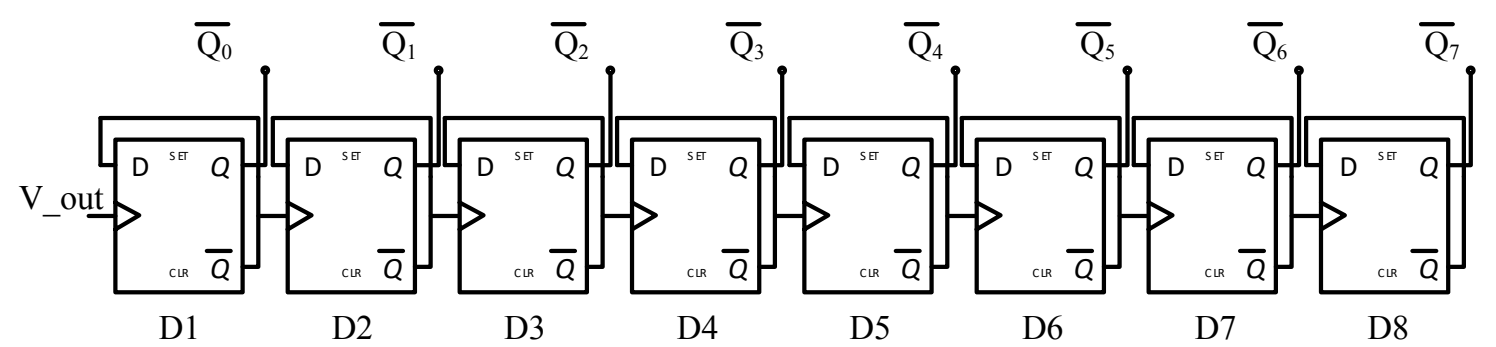

Fig. 3.11: Diagram of an 8-bit digital counter 
This configuration is not only simpler but also improves the dynamic range. For example, in this work, the total number of loop cycles is the sum of the two loop counter outputs $N_{1}$ and $N_{2}$ as mentioned before. Each of them is an 8-bit binary code and their sum is 9 bits. It also should be mentioned that the circulating signal has previously been divided once by the differential MCML delay chain. Consequently, the dynamic range of this TDC core is two times that of a conventional looped TDC counted by a dual-edge triggered counter. In addition, the DETSE requires twice as many devices to a simple rising edge triggered element [35]. A pair of counters is used to replace the dual-edge triggered counter in this work. That also means no extra cost in the area and as such the power consumption is involved by using this configuration.

\subsubsection{PISO-Register}

The counters discussed previously output data in parallel 8-bit binary form which requires at least 8 pins to probe it. For the convenience of observing the measurement results, a PISO-register is designed to convert the parallel 8-bit binary output from the counters to a serial format.

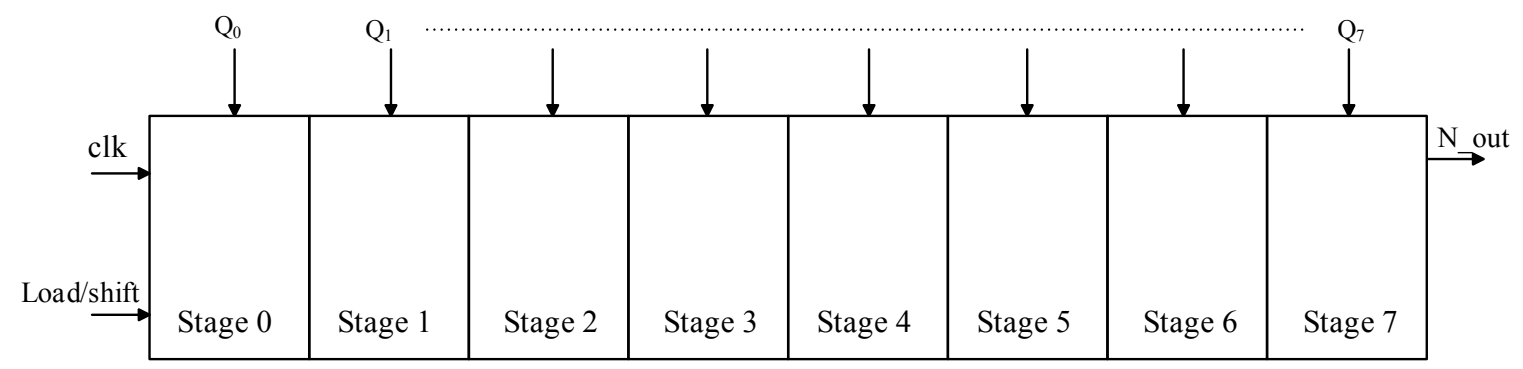

Fig. 3.12: Diagram of a parallel-in-serial-out shift register

As shown in Figure 3.12, 8-bit counter outputs are injected to all stages simultaneously. Then the register stores the data, shifts it on each clock edge and delays it 
by the number of stages multiplied by the clock period. By serial format, we mean that the data bits are presented sequentially in time on a single wire or connection.

With the data conversion process discussed above, 8 parallel outputs are reduced to one. This configuration significantly saves output pins and also the bonding pads of the chip prototype. 


\section{Chapter 4}

\section{Circuit Design and Implementation}

This chapter describes the MCML-based TDC design and implementation in detail. The schematic and layout of each block are presented. Design considerations and improvement techniques are discussed in this chapter.

The TDC prototype presented by this work was implemented in a mixed-signal $0.13 \mu \mathrm{m}$ CMOS technology. The TDC prototype was designed starting with a feasible analysis. Then schematics were constructed and converted into layouts. Each layout was examined with design-rules-checking (DRC) and Layout-versus-schematic (LVS).

The novel TDC prototype consists of the following blocks:

- A 4-steps switchable MCML-Buffer based TDC core,

- An output driving buffer array used to drive the 12-bit thermometer code,

- 2 complementary 8-bit counters with sample array attached,

- 2 PISO-shift registers. 


\subsection{Logic Gate Library Design}

The switchable MCML-based TDC uses basic logic cells and sample elements specifically created for this work. The Canadian Microelectronic Corporation (CMC) provides a fundamental ARM standard cell library named the $130 \mathrm{~nm}$ IBM CMRF8SF library. This means the peripheral circuits (loop counter, PISO-shift register, driving buffers) which are made of conventional logic gates are possible to be constructed with the synthesized and P\&R design flow. Unfortunately, the ARM standard cell library does not include GDSII views. This means the standard cells are represented by black boxes and their internal operation cannot be simulated in Cadence. Therefore, a custom designed digital cell library is used. An incidental advantage is that the gates can be designed specifically to high performance requirements of some of the major blocks. All of the gates are designed with the same rail-to-rail layout height, allowing all of the power supply rails and ground rails to be connected easily.

\subsubsection{INV1}

INV1 is an inverter. This gate is used in the 8-bit rising-edge counter. The outputs of the counter need to be logically reversed in order to be correctly injected into the PISO-shift register. The schematic and layout are shown in Figure. 4.1. In order to equalize the rising and falling time of the inverter, The ratio of widths is chosen to be 3:1. The default minimum channel length for each type of transistors is $120 \mathrm{~nm}$ for this CMOS technology. Therefore, the pfet width is set to be $3 \mu \mathrm{m}$ and the length is $120 \mathrm{~nm}$. The nfet width is $1 \mu \mathrm{m}$ and the length $120 \mathrm{~nm}$. For the rest of this work, the width of transistors which is wider than $2 \mu \mathrm{m}$ will be split into multi-fingers. This is for the purpose of keeping all building blocks share the same rail-to-rail height. The subc is the substrate contact in the library. - 

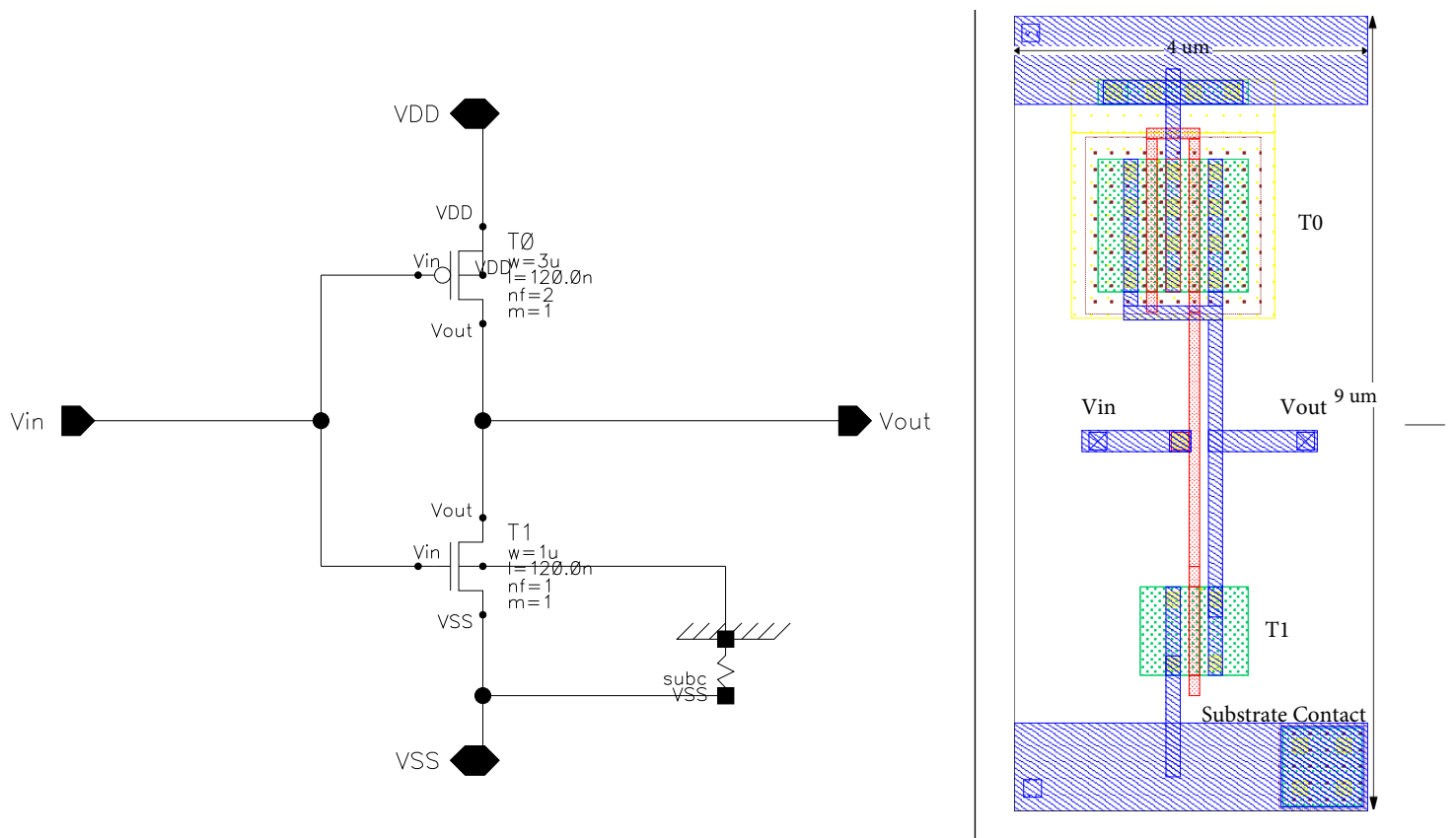

Table 4.1: Inverter INV1 and its corresponding layout. T0 is $3 \mu \mathrm{m}$ wide and 120 $\mathrm{nm}$ long. $\mathrm{T} 1$ is $1 \mu \mathrm{m}$ wide and $120 \mathrm{~nm}$ long.

\subsubsection{NAND2D1}

The two input NAND gate implementation is shown in Figure 4.1. This gate was used in the Ring Closer block. As discussed above, the ring closer forms the delay path into a loop and is likely to bring imbalance into the delay chain. Hence, the NAND gate should be carefully designed to minimize propagation delay. Therefore, a specific global optimization environment is created in order to optimize the performance of this gate. First, the lengths of all transistors in this gate are set to be $120 \mathrm{~nm}$. Then the widths of pfets and nfets are set to be variables individually. By presetting each variable with, certain swing range, step size (e.g. $180 \mathrm{~nm}: 20 \mathrm{~nm}: 5 \mu \mathrm{m}$ ) and objective parameters (e.g. propagation delay $<10 \mathrm{ps}$ ), the optimization tool will simulate all expressions iteratively and provide all suitable design points.

For the purpose of avoiding the delay asymmetry, the propagation delay of the NAND gate is optimized to achieve the same delay of a delay cell. Hence, the pfet 
transistors were sized with a width of $4 \mu \mathrm{m}$ and length of $120 \mathrm{~nm}$. The nfet transistors width is $3.04 \mu \mathrm{m}$ and the length is $120 \mathrm{~nm}$. Both the nfet and pfet are layed out with multiple fingers.

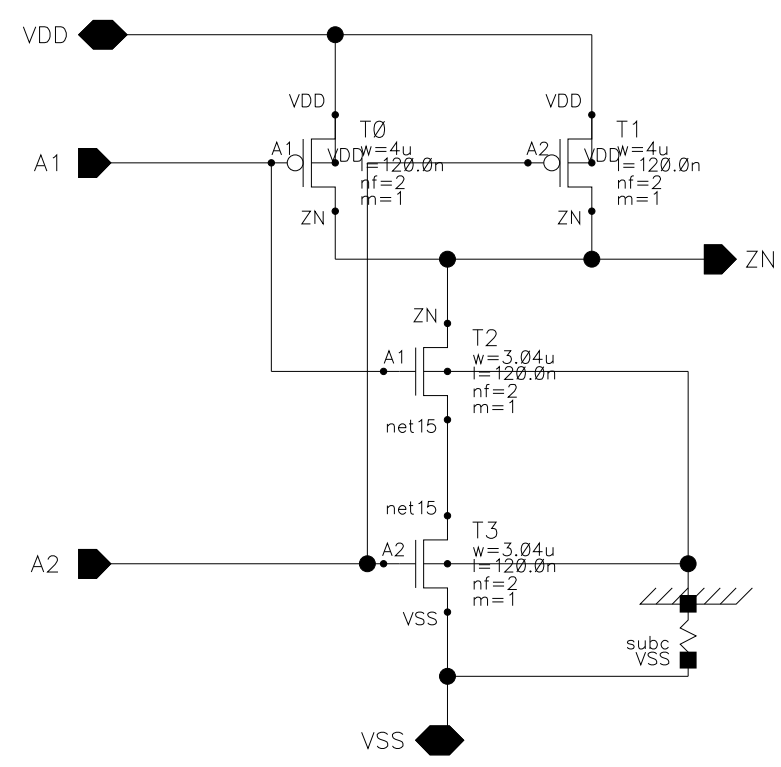

(a) schematic

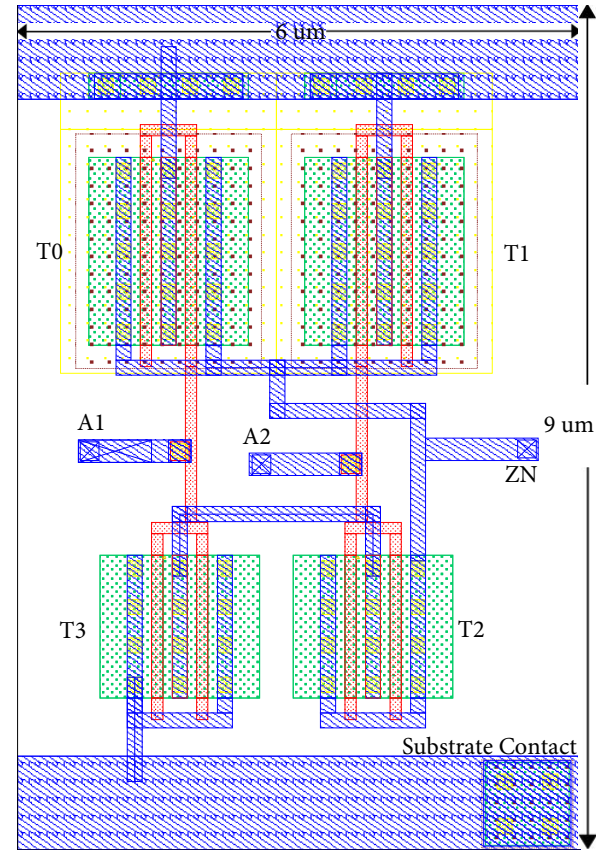

(b) layout

Fig. 4.1: NAND2D1 gate and its corresponding layout. The widths of T0 and T1 is $4 \mu \mathrm{m}$ with 2 fingers and their lengths are $120 \mathrm{~nm}$. T2 and T3 are $3.04 \mu \mathrm{m}$ wide with 2 fingers and $120 \mathrm{~nm}$ long. 


\subsubsection{BufferD1}

For external testing, the signal of each output needs to be strengthened in order to drive the large capacitance of the bonding pads. Therefore, a buffer array is required. The buffer array is formed by a two inverter-based buffer gates connected in series and increasing in size. Figure 4.2 shows an example of the pad driving buffer which was developed based on the classic [36].

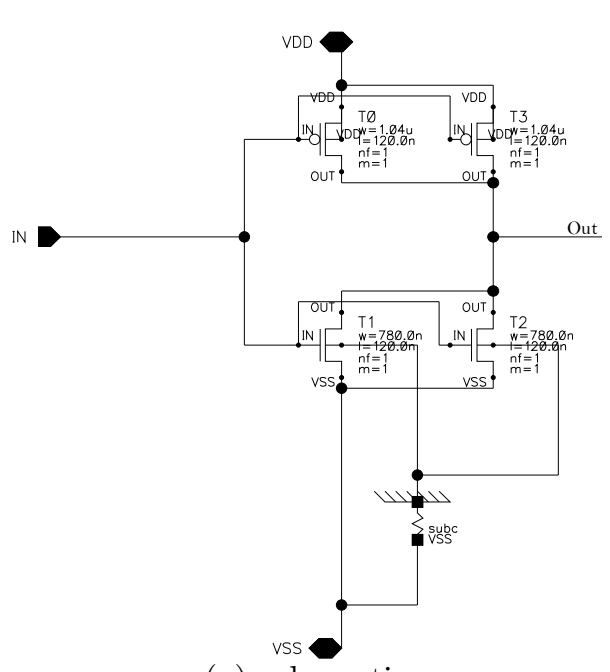

(a) schematic

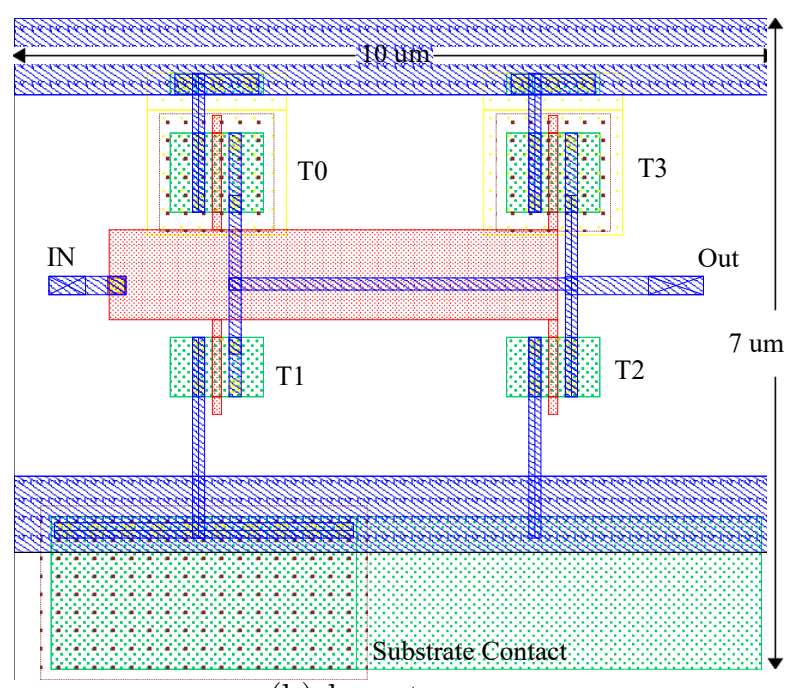

(b) layout

Fig. 4.2: An example of a Buffer gate and its corresponding layout. The widths of $\mathrm{T} 0$ and T3 are $1.04 \mu \mathrm{m}$ and lengths are $120 \mathrm{~nm}$. T1 and T2 are $780 \mathrm{~nm}$ wide and $120 \mathrm{~nm}$ long.

A basic building block is comprised of two simple inverters. The buffer is used just in the driving buffer chain in this work. Its charging and discharging character is unnecessary to be balanced. Therefore, the ratio of transistors widths is chosen from the standard cell in library. The pfet width is $1.04 \mu \mathrm{m}$ and the nfet width is $780 \mathrm{~nm}$. Both the nfet and pfet share the same channel length of $120 \mathrm{~nm}$. This buffer is the smallest in a driving buffer chain. 


\subsubsection{DFF1}

The D type Flip-Flop is a crucial component. It serves not only as the sample elements in the TDC core block, but also as a basic building block in both the 8-bit counter and the PISO Shift Register. Furthermore, the output of the counter requires a sample and hold $(\mathrm{S} / \mathrm{H})$ circuit to be able to stop the counting result and inject the results into the shift register at a certain point of time. The DFF1 is a D type Flip-flop specifically designed for this work. The schematic diagram of the DFF1 is shown in Figure. 4.3. The layout is shown in Figure. 4.4.

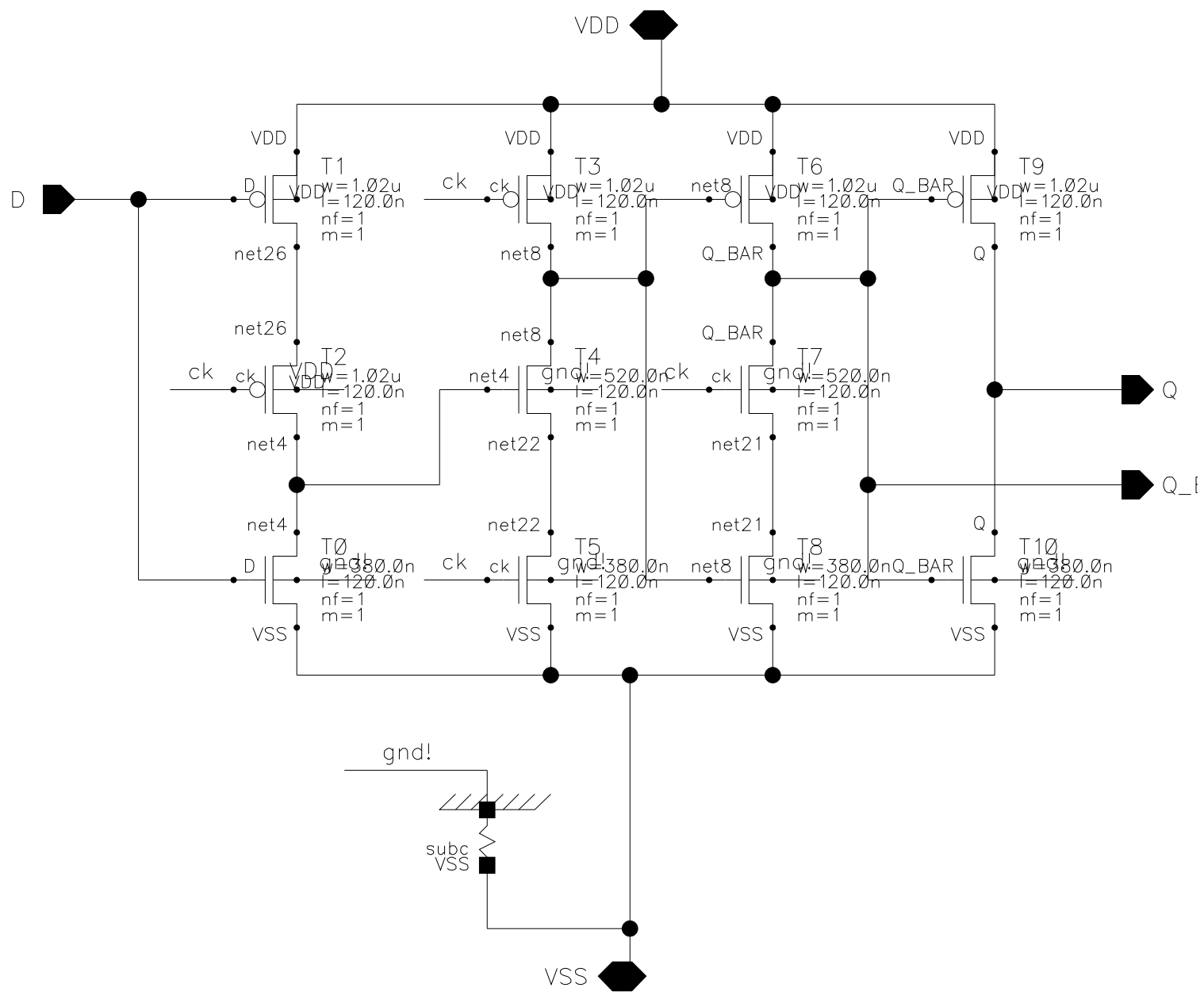

Fig. 4.3: The schematic diagram of the D type Flip-Flop. 


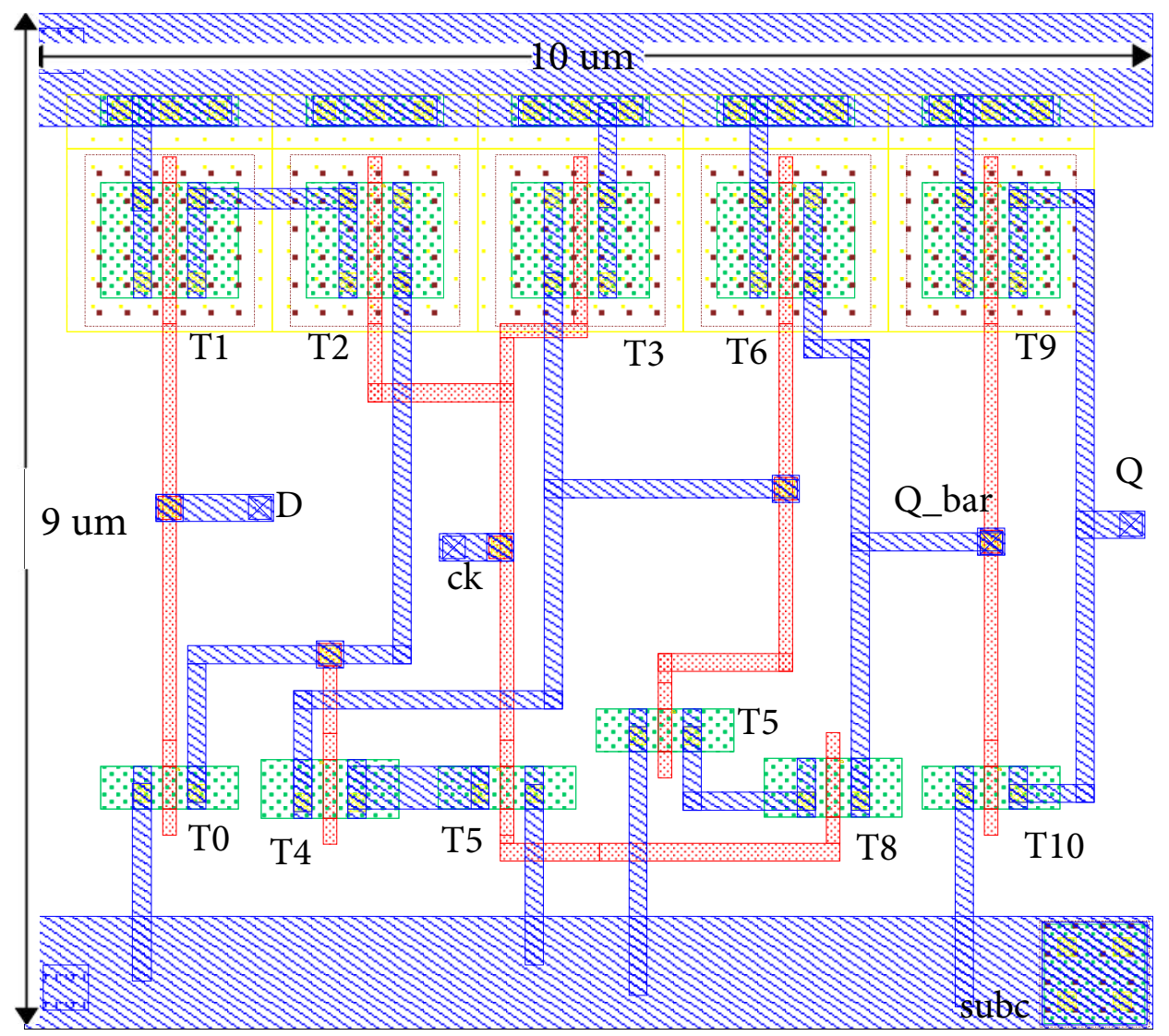

Fig. 4.4: The layout diagram of the D type Flip-Flop.

The sizes of the transistors in Figure. 4.3 are shown in Table. 4.2 
Table 4.2: The sizes of each transistor in Figure. 4.3

\begin{tabular}{|l|c|c|c|c|}
\hline Transistor Name & Type & Width & Length & Number of Fingers \\
\hline T0 & nfet & $380 \mathrm{~nm}$ & $120 \mathrm{~nm}$ & 1 \\
\hline T1 & pfet & $1.02 \mu m$ & $120 \mathrm{~nm}$ & 1 \\
\hline T2 & pfet & $1.02 \mu m$ & $120 \mathrm{~nm}$ & 1 \\
\hline T3 & nfet & $520 \mathrm{~nm}$ & $120 \mathrm{~nm}$ & 1 \\
\hline T4 & pfet & $1.02 \mu m$ & $120 \mathrm{~nm}$ & 1 \\
\hline T5 & pfet & $380 \mathrm{~nm}$ & $120 \mathrm{~nm}$ & 1 \\
\hline T6 & pfet & $1.02 \mu \mathrm{m}$ & $120 \mathrm{~nm}$ & 1 \\
\hline T7 & nfet & $520 \mathrm{~nm}$ & $120 \mathrm{~nm}$ & 1 \\
\hline T8 & nfet & $380 \mathrm{~nm}$ & $120 \mathrm{~nm}$ & 1 \\
\hline T9 & pfet & $1.02 \mu \mathrm{m}$ & $120 \mathrm{~nm}$ & 1 \\
\hline T10 & nfet & $380 \mathrm{~nm}$ & $120 \mathrm{~nm}$ & 1 \\
\hline
\end{tabular}

\subsubsection{SWMCML-Buffer}

The SWMCML-Buffer is the optimized switchable-buffer gate discussed previously. The schematic of the SWMCML-Buffer is shown in Figure 4.5.

Passive resistors occupy a large amount of layout area. To minimize the area, transistors $T 0, T 1, T 2, T 3$, and T8, T9, T10,T11 which are P-channel MOSFETs form the active loads/pull-up networks. The transistor groups T0 to T3 and T8 to T11 were sized to be the same (T0 same as T8, T1 same as T9, etc.). In total, 4 pairs of pfet loads form the 4 different operating points of the delay cell. The switching activity is achieved by manipulating the gate to source voltages of each pair of loads. As shown in Figure 4.5, four different reference voltages are $V R P_{0}, V R P_{1}, V R P_{2}$ and $V R P_{3}$. With four different pfet widths, the equivalent resistance of each pair is also different. Therefore, the MCML-Buffer gate could operate with four different propagation delays. The sizes of all the transistors in the MCML gate are presented 


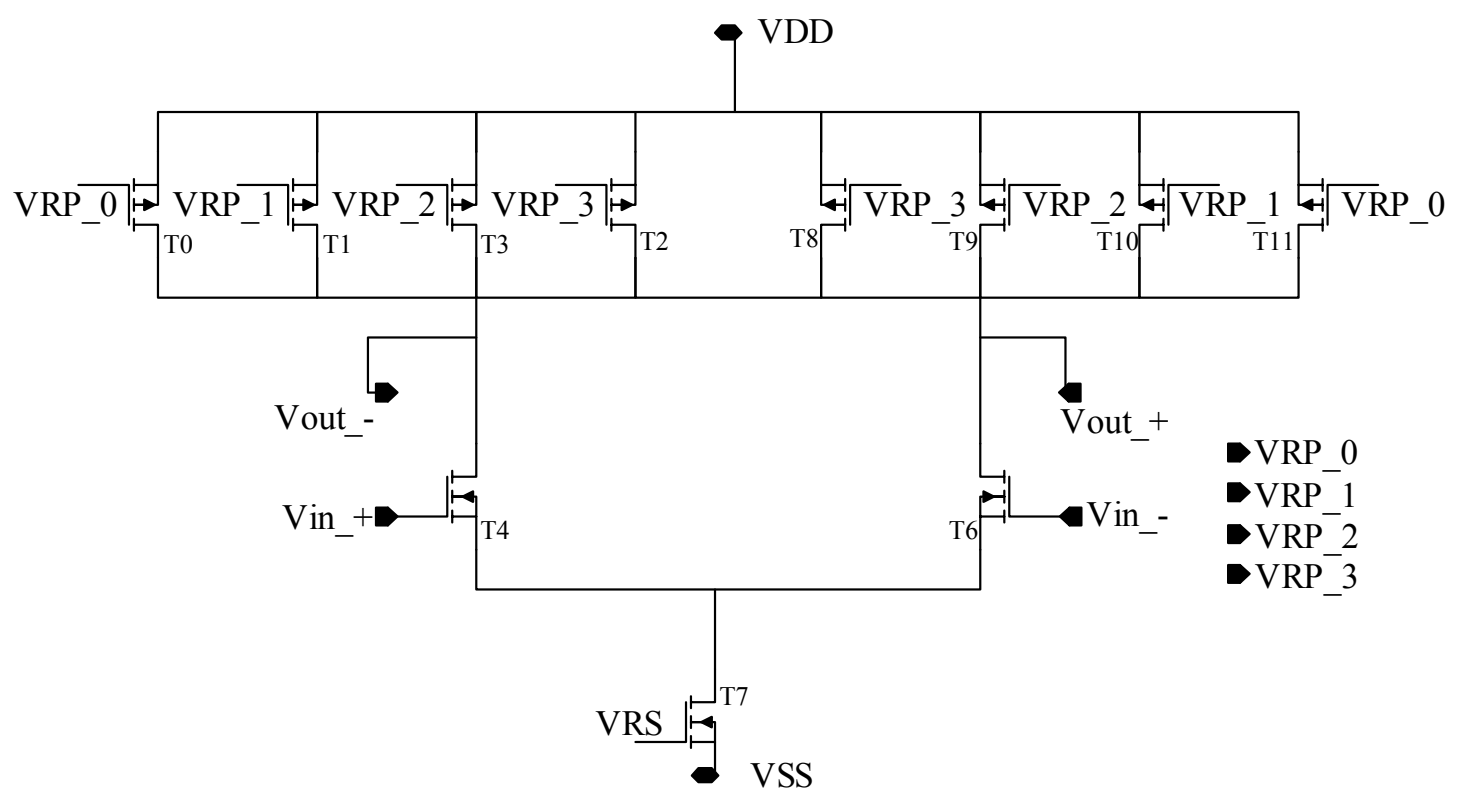

Fig. 4.5: The schematic diagram of the four steps switchable MCML Buffer gate

in Table 4.3.

Table 4.3: The sizes of each transistor in Figure 4.5

\begin{tabular}{|l|c|c|c|c|}
\hline Transistor Name & Type & Width & Length & Number of Fingers \\
\hline T0 & pfet & $1.2 \mu m$ & $120 \mathrm{~nm}$ & 1 \\
\hline T1 & pfet & $3.08 \mu m$ & $120 \mathrm{~nm}$ & 2 \\
\hline T2 & pfet & $2.28 \mu m$ & $120 \mathrm{~nm}$ & 2 \\
\hline T3 & pfet & $1.9 \mu m$ & $120 \mathrm{~nm}$ & 1 \\
\hline T4 & nfet & $4.6 \mu \mathrm{m}$ & $120 \mathrm{~nm}$ & 4 \\
\hline T6 & nfet & $4.6 \mu \mathrm{m}$ & $120 \mathrm{~nm}$ & 4 \\
\hline T7 & nfet & $64 \mu \mathrm{m}$ & $120 \mathrm{~nm}$ & 32 \\
\hline T8 & pfet & $1.9 \mu \mathrm{m}$ & $120 \mathrm{~nm}$ & 1 \\
\hline T9 & pfet & $2.28 \mu \mathrm{m}$ & $120 \mathrm{~nm}$ & 2 \\
\hline T10 & pfet & $3.08 \mu \mathrm{m}$ & $120 \mathrm{~nm}$ & 2 \\
\hline T11 & pfet & $1.2 \mu \mathrm{m}$ & $120 \mathrm{~nm}$ & 1 \\
\hline
\end{tabular}

$T 4$ and $T 6$ form the differential pair and work as the switching component of the 
MCML gate. When the differential input is applied to the logic block, the current is steered between the left and right branches. The output nodes are either pulled high or low based on the input signal. As this TDC design needs an inverting buffer stage and this building block is an inverter, the output of one stage are cross-coupled to the input of the next delay cell. As discussed previously, the sinking current is driven by the tail nfet. In this schematic, the bottom n-type transistor $T 7$ plays this role. It was designed to have a extremely large channel width with a multi-finger configuration in order to drive a large bias current [37].

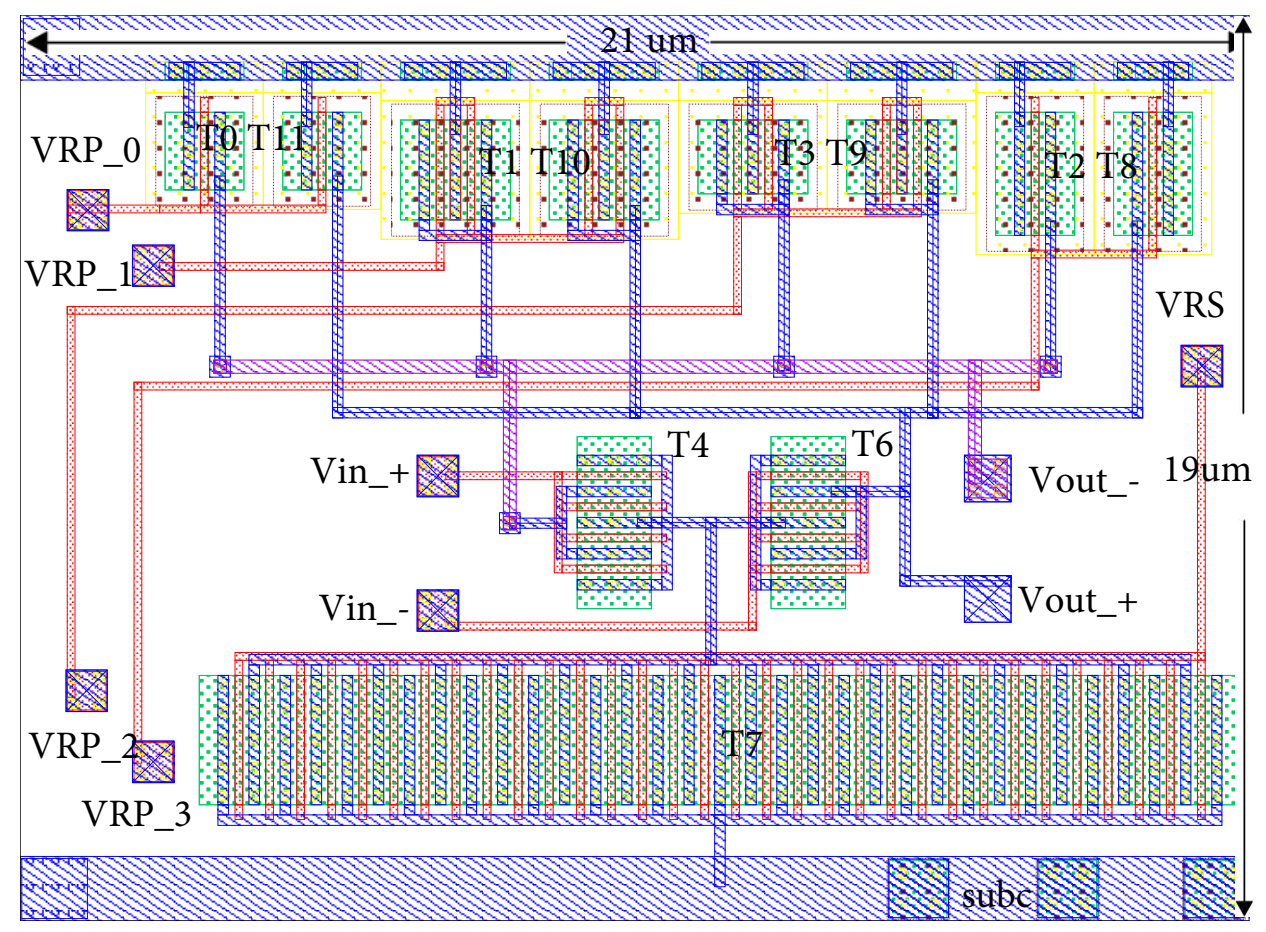

Fig. 4.6: The layout diagram of the 4 steps switchable MCML Buffer gate

The layout of the MCML-Buffer is shown in Figure 4.6. The width of each transistors was chosen by the global and local optimization tool in the Cadence analog design environment (ADE) . The lengths of the transistors in this logic are set to be the minimum in order to increase their operational speed. All pull-up transistor pairs 
are placed on top of the layout. In order to compensate the processing variation, the transistors with the same size are placed adjacently [38]. 


\subsection{TDC Implementation}

With all of the circuits listed above, the switchable TDC prototype can be implemented. In the next subsection, each module implementation will be discussed.

\subsubsection{Architecture}

Figure 4.7 shows the overall architecture of the novel switchable TDC design. Each block and its I/O pins are shown on the diagram and will be presented in detail in this section. In order to reduce the number of the overall bond pads, all devices in this work are operating with the same power supply of $1.5 \mathrm{~V}$. 


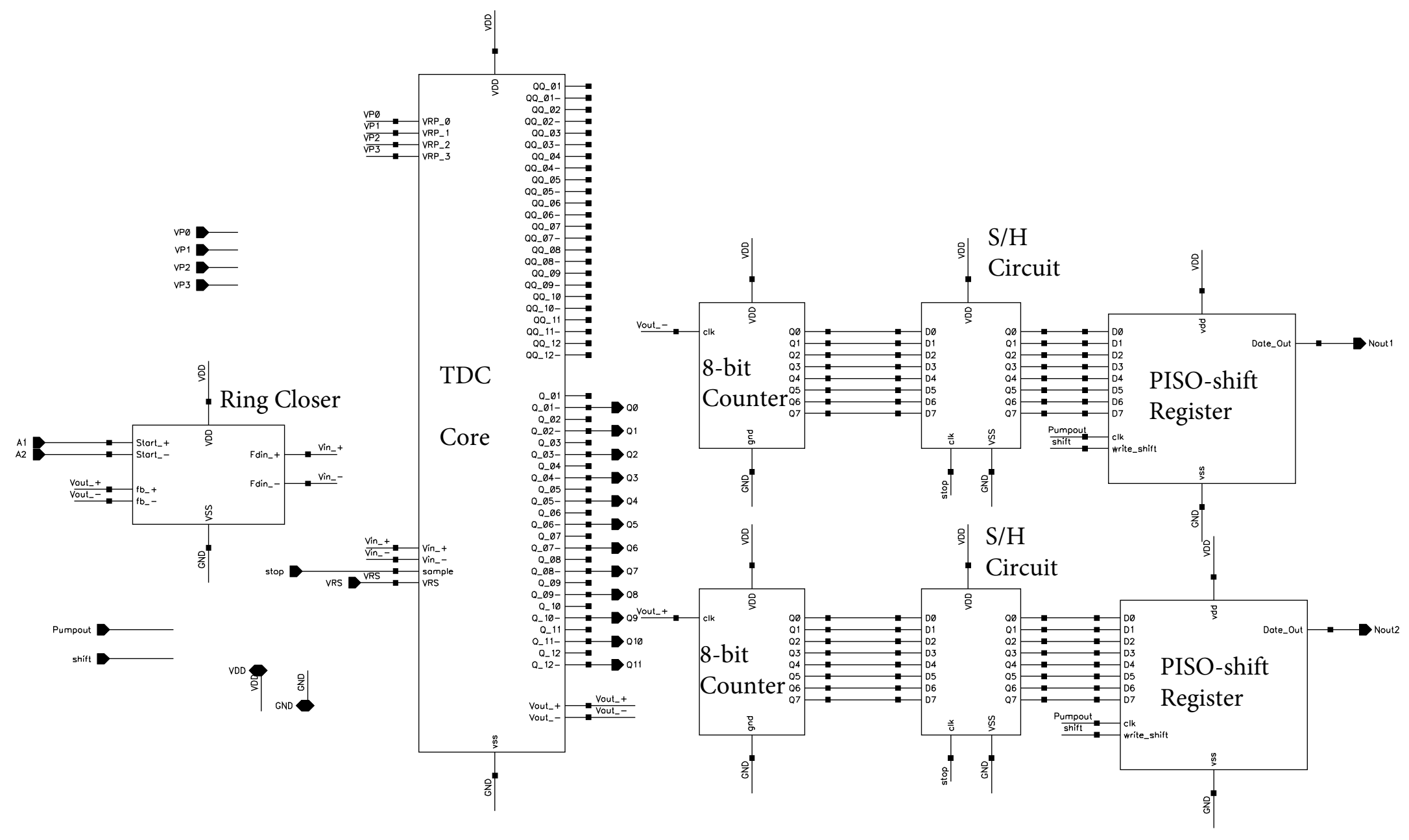

Fig. 4.7: The overall block diagram of the switchable TDC design 


\subsubsection{Fine And Coarse TDC Core Implementation}

The switchable MCML-Buffer is the most important component of the TDC core. This is because the most critical parameter of TDC (resolution) is specifically determined by the propagation delay of the MCML-Buffer gate. The schematic of this block as well as the corresponding layout are shown in Figure 4.8.

From the schematic view, the TDC core circuit is the combination of 12 stages cascaded in a row. The differential start signal is injected into the input pins $V i n_{+}$ and Vin - of the first stage. Each stage consists of a switchable MCML-Buffer and two Flip-Flops (FFs). The differential outputs of the MCML-Buffer load the two FFs as well as the MCML-Buffer of the next stage. At the end of this delay chain, the differential output pins are placed. The loop is closed by the Ring Closer block which will be presented in the next subsection. There are two FF arrays on the schematic. The lower array is chosen to output the 12-bit thermometer-code and the upper array is the complementary code. Also, each FF has two differential outputs with the opposite polarity. Hence, it is not necessary to observe both of them. Eventually the output pins on the bottom are selected, which are $Q_{01}-Q_{12}$. 

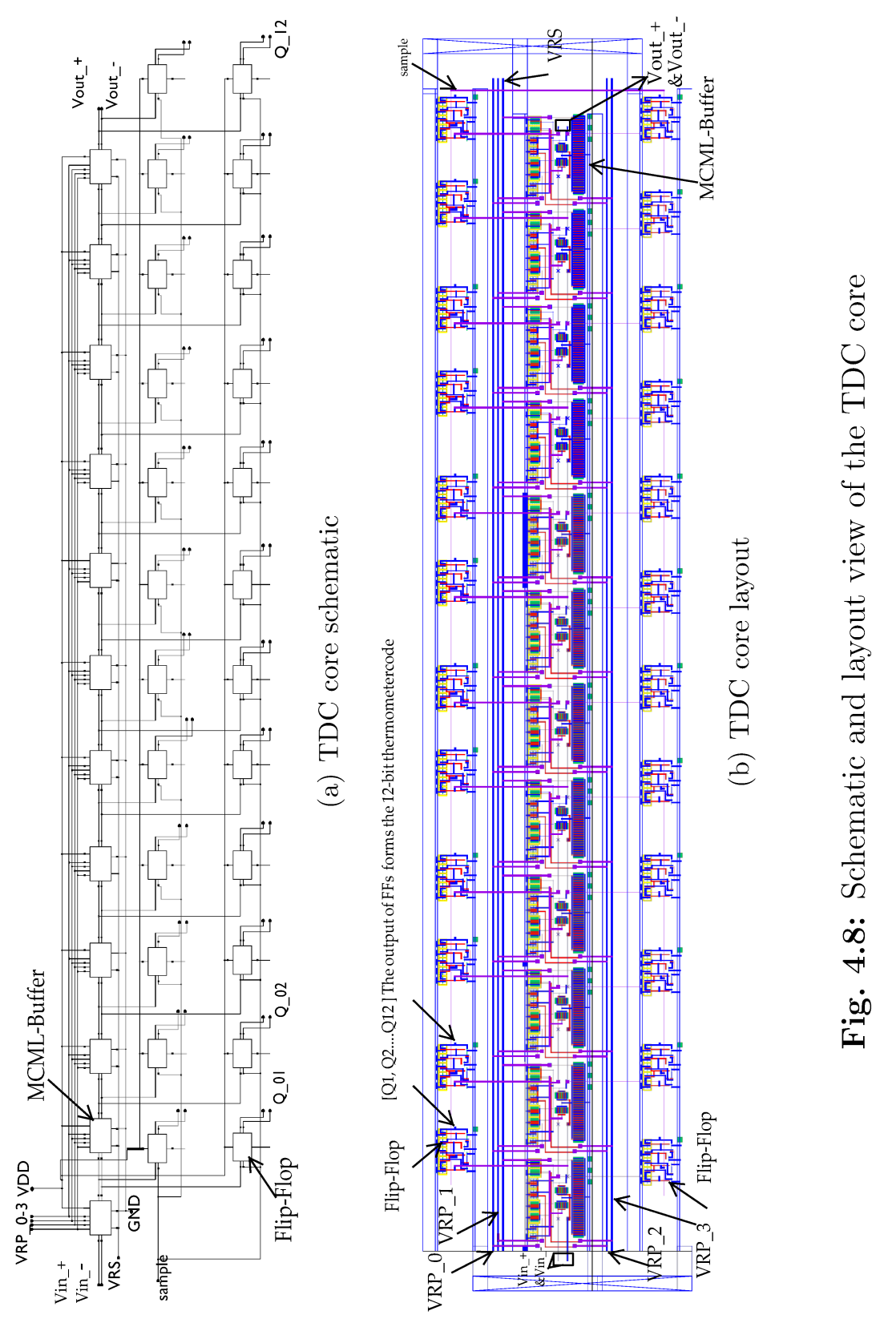
The delay chain discussed previously is a delay sensitive circuit. Delay imbalance should be avoided in order to minimize the DNL. For example, each stage should be placed with the same patch (gap between two of the stages). Also, the connection between the two stages should be minimized for the purpose of reducing parasitic capacitance.

The hierarchical symbol which represents the TDC core circuit is shown in Figure 4.9 .

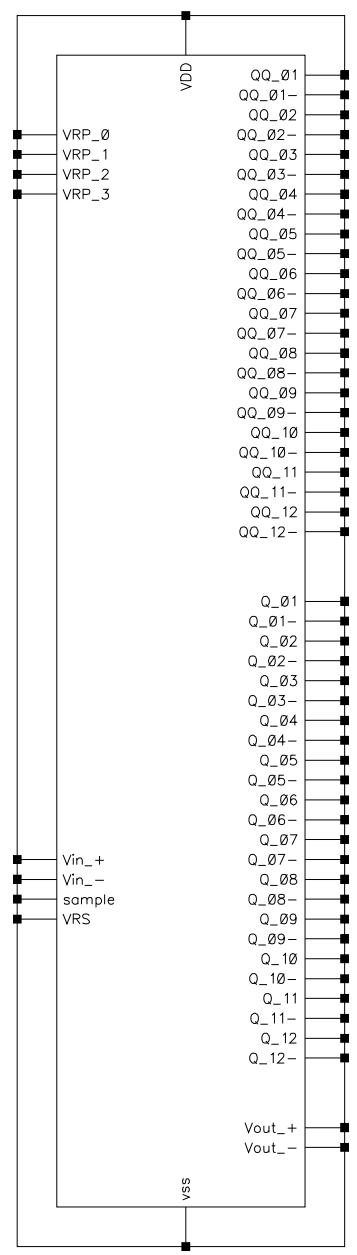

Fig. 4.9: The diagram of the TDC core symbol 
Table 4.4: Pins Descriptions in Figure. 4.9

\begin{tabular}{|c|c|c|}
\hline Pin Name & Type & Pin Description \\
\hline VDD & $\mathrm{P}$ & 1.5V Power Supply Pin \\
\hline VSS & $\mathrm{P}$ & Supply Ground Pin \\
\hline$V R P_{0}$ & $\mathrm{P}$ & Active Load Control \\
\hline$V R P_{1}$ & $\mathrm{P}$ & Active Load Control \\
\hline$V R P_{2}$ & $\mathrm{P}$ & Active Load Control \\
\hline$V R P_{3}$ & $\mathrm{P}$ & Active Load Control \\
\hline sample & $\mathrm{P}$ & Stop Signal Input Pin \\
\hline VRS & $\mathrm{P}$ & Biasing Current Control \\
\hline $\operatorname{Vin}_{+}$ & I & Differential Input 1 \\
\hline Vin $_{-}$ & I & Differential Input 2 \\
\hline$Q_{01}-Q_{12}$ & $\mathrm{O}$ & 12-bit Thermometer Coder Output \\
\hline$Q_{01-}-Q_{12-}$ & $\mathrm{O}$ & Complementary 12-bit Thermometer Code Output \\
\hline Vout $_{+}$ & $\mathrm{O}$ & Differential Output 1 \\
\hline Vout_ & $\mathrm{O}$ & Differential Output 2 \\
\hline
\end{tabular}

The pin information is listed in Table 4.4. 


\subsubsection{Ring Closer Implementation}

Figure 4.10 shows the hierarchical symbol of the Ring Closer block used to form the TDC core delay chain into a loop. This block is made up of two NAND gates and because this block is embedded in the delay loop, it needs to be specifically optimized to have the same propagation delay of the MCML-buffer. This helps to avoid the involvement of the delay imbalance.

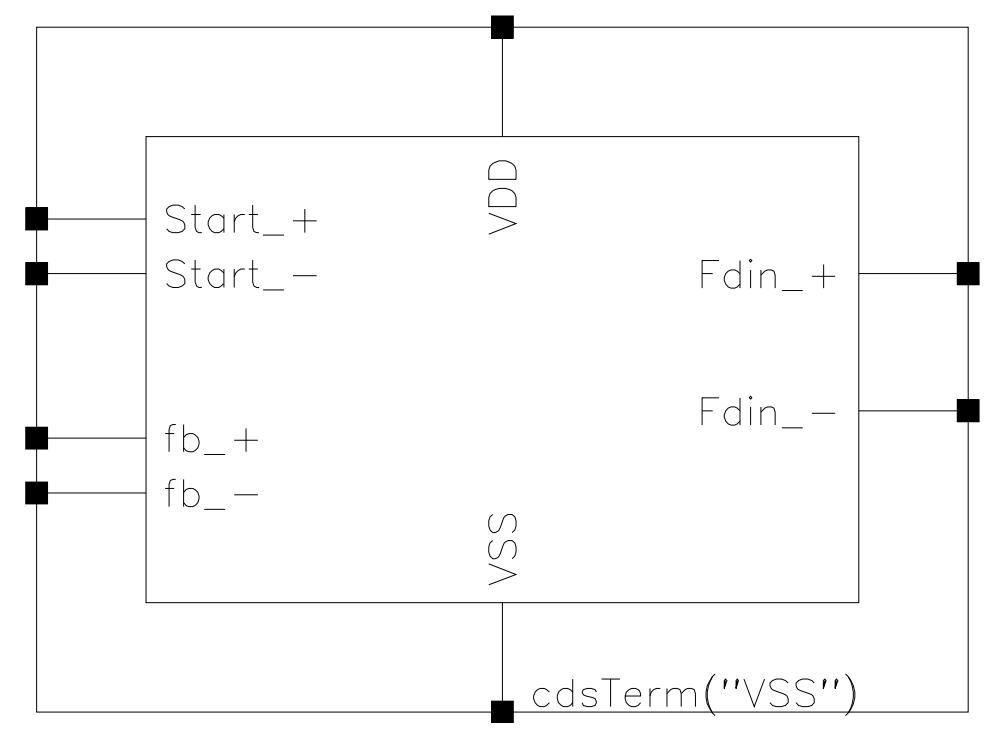

Fig. 4.10: Ring Closer Implementation Symbol

The pin information is illustrated in Table 4.5.

As in the other blocks, the Ring Closer uses a $1.5 \mathrm{~V}$ power supply. The Ring Closer is inspired by the conventional SR latch [39]. The schematic of the Ring Closer is depicted in Figure 4.11. Note that the feedback signal is cross coupled into the pins $f b_{-}$and $f b_{+}$. This means the logic level is flipped at the beginning of each cycle. Since our delay chain consists of 12 stages, the logic state will not change whether the MCML-Buffer works as an Inverter or a Buffer. Hence, the cross coupled connection 
Table 4.5: Pins Descriptions in Figure. 4.10

\begin{tabular}{|c||c|c|}
\hline Pin Name & Type & Pin Description \\
\hline Start $_{+}$ & I & Differential Input 1 \\
\hline Start $t_{-}$ & I & Differential Input 2 \\
\hline$f b_{+}$ & I & Feedback signal Input 2 \\
\hline$f b_{-}$ & I & Feedback signal Input 1 \\
\hline VDD & P & 1.5V Power Supply Pin \\
\hline VSS & P & Supply Ground Pin \\
\hline Fdin & O & Looped Signal Feed-in Pin 1 \\
\hline Fdin & O & Looped Signal Feed-in Pin 2 \\
\hline
\end{tabular}

of the Ring Closer is very important.

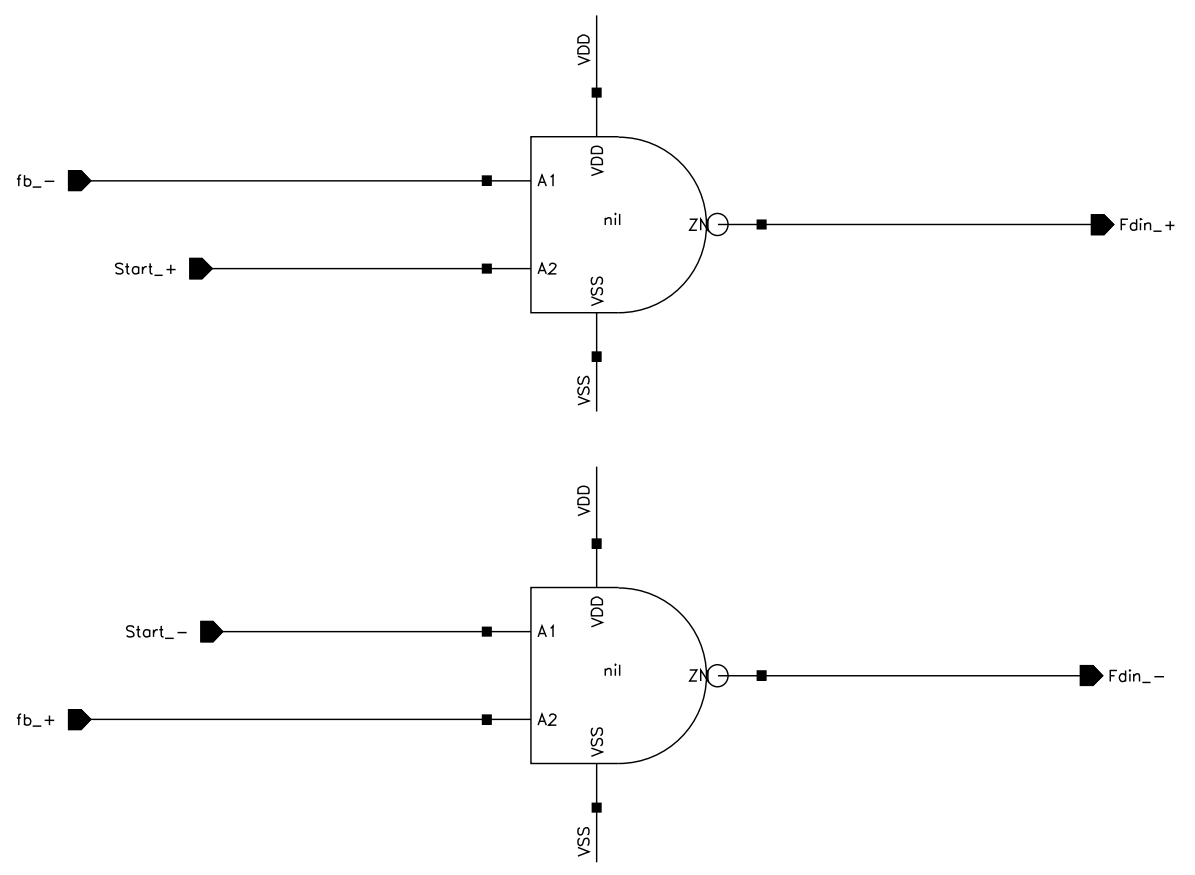

Fig. 4.11: The schematic of the Ring Closer. This block is made up of two NAND gates. For each NAND one input is for the start signal injection and the other one is for the feed-back signal. 
The layout of the Ring Closer block is shown in Figure 4.12. For the MCMLBuffer layout view in Figure 4.6 discussed previously, the differential signal injection pin is vertically placed. Therefore, the two NAND gates in the Ring Closer are placed vertically for the purpose of delay imbalance reduction. This means that for the two complementary paths of the differential delay line, each of them must have the fully symmetric connection compared with the other one. The VDD and VSS rails are connected separately.

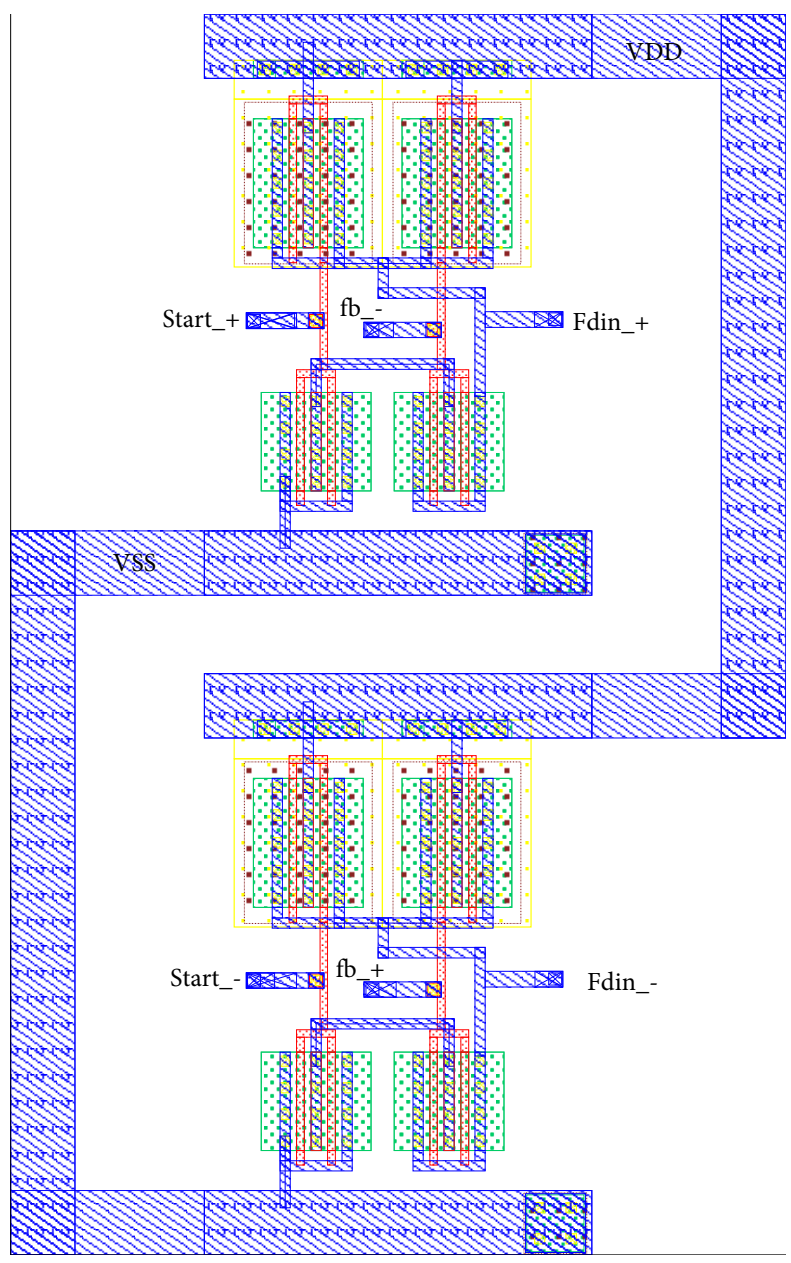

Fig. 4.12: The layout of the Ring Closer block 


\subsubsection{Loop Counter Implementation}

This block is the 8-bit rising edge counter discussed in Chapter 3. It is used to count both the rising edge in the upper path and the complementary rising edge in the lower path of the delay chain. At the point in time the stop signal arrives, the sum of counting binary number represent how many cycles the start event has traversed in the delay loop. The hierarchical symbol of the Loop Counter is shown in Figure 4.13.

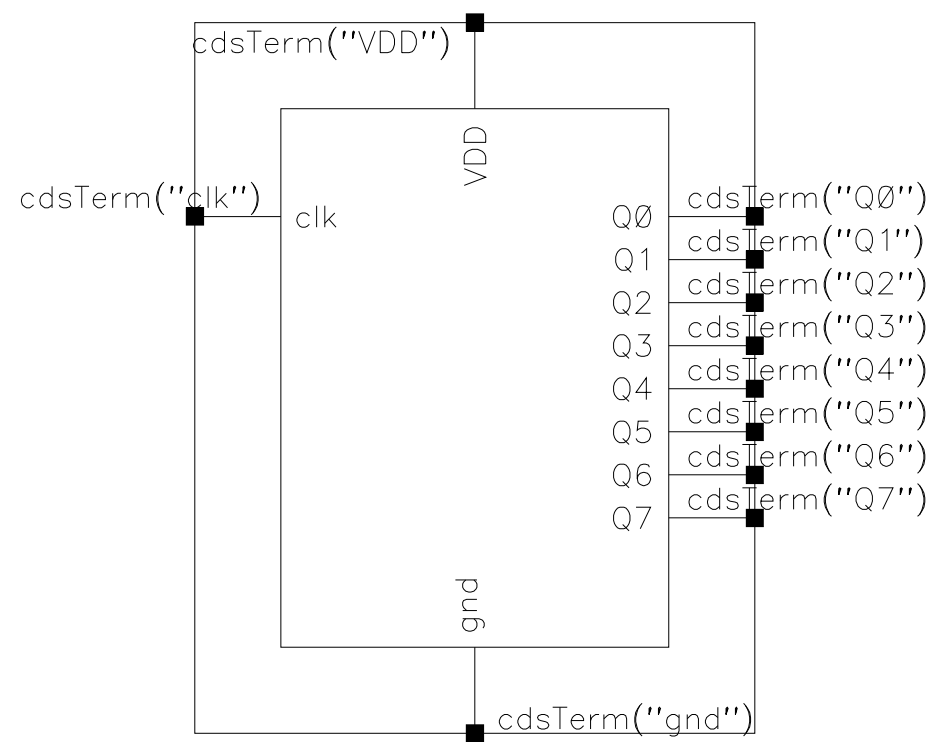

Fig. 4.13: 8-bit rising edge counter hierarchical Symbol

Table 4.6 shows all of the pin information for this cell. The $c l k$ pin is the input where the circulating signal from the TDC core block is injected. The output pins from $Q 0$ to $Q 7$ form the 8-bit parallel binary code. As in the other modules in this work, the counter uses $1.5 \mathrm{~V}$ power supply.

The schematic diagram is shown in Figure 4.14. The counter is formed with 8 stages. Each stage is comprised of a FF and an output inverting element (an Inverter in this case). For each FF, the positive output is connected with the the input clk 
Table 4.6: Pins Descriptions in Figure. 4.13

\begin{tabular}{|c||c|c|}
\hline Pin Name & Type & Pin Description \\
\hline clk & I & Circulating Signal Input Pin \\
\hline$Q 0-Q 7$ & O & 8-bit Parallel Binary Output Pin \\
\hline VDD & P & 1.5V Power Supply Pin \\
\hline VSS & P & Supply Ground Pin \\
\hline
\end{tabular}

of the next stage and the input of the inverter. Only the input $c l k$ of the first stage is applied with the signal provided by the TDC core block. Eventually, the required 8-bit binary code is combined with all the inverter's outputs.

The layout diagram of the Loop Counter is shown in Figure 4.15. All of the gates are placed in a row. With this configuration, all the gates are able to share one power supply rail and one ground rail. Furthermore, the output pad of each FF is placed with the same patch (distance between two FFs). This makes the connections between these outputs and the next blocks the same length, which helps avoid the delay imbalance. 


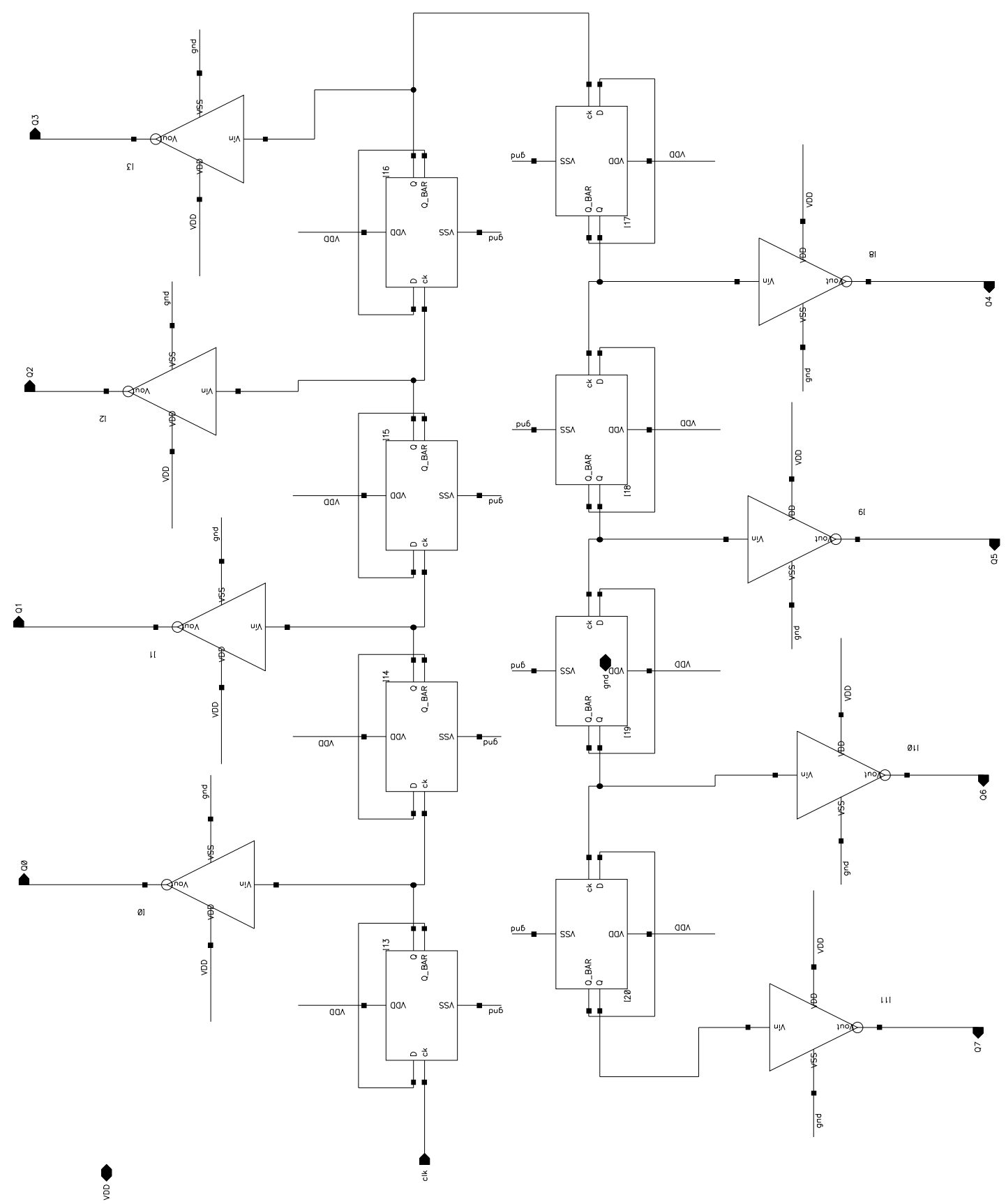

Fig. 4.14: The schematic of the Loop Counter. This block is made up of 8 stages. Each stage consists of a Flip-Flop and a logic shifting element. 


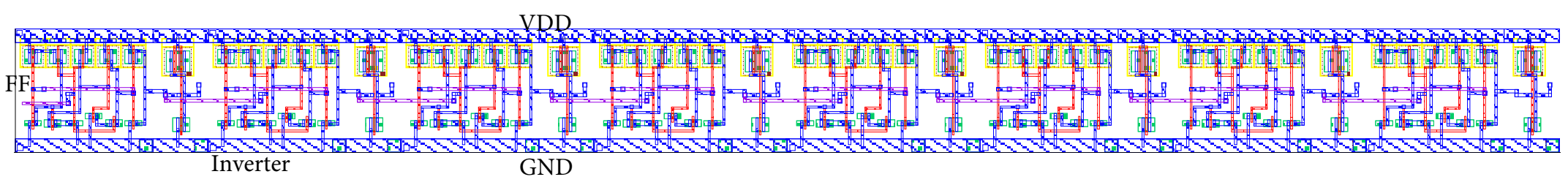

Fig. 4.15: The layout of the Loop Counter. All gates are placed in a row. 


\subsubsection{State Freeze Circuit Implementation}

The Loop Counter discussed above counts the circulation of the start signal constantly. It cannot stop the counting process at a certain point in time. However, at the rising edge of the stop signal, both the number of full cycles $N$ and the thermometer code are required. Thus, an additional block is used to sample the 8-bit counter when the stop signal occurs. The hierarchical symbol of this block is shown in Figure 4.16.

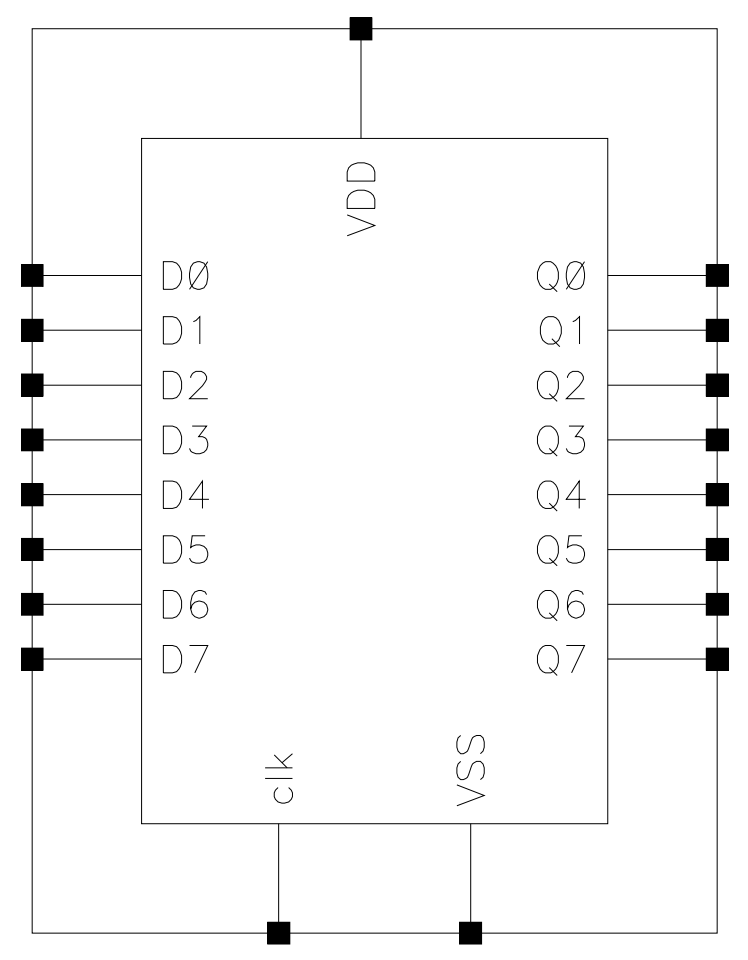

Fig. 4.16: The hierarchical symbol of the State Freeze block with all pins

Table 4.7 shows the pin description of the symbol in Figure 4.16. The power supply $V D D$ and ground VSS are the same as all other blocks. An 8-bit bus transfers the data from the Loop Counter to the input pins from $D 0$ to $D 7$ in this circuit. When the stop signal triggers the $c l k$ pin, the counting result is frozen and outputted by 
Table 4.7: Pins Descriptions in Figure. 4.16

\begin{tabular}{|c||c|c|}
\hline Pin Name & Type & Pin Description \\
\hline clk & I & Stop Signal Injection Pin \\
\hline$D 0-D 7$ & I & 8-bit Parallel Binary Input Pin \\
\hline$Q 0-Q 7$ & O & 8-bit Parallel Binary Output Pin \\
\hline VDD & P & 1.5V Power Supply Pin \\
\hline VSS & P & Supply Ground Pin \\
\hline
\end{tabular}

the pins from $Q 0$ to $Q 7$

The schematic of the State Freeze circuit is shown in Figure 4.17. It contains 8 FFs and each FF is in charge of sampling one bit of the counter's outputs. All devices are triggered by the same $c l k$. It should be mentioned that for design that contains a large number of devices enabled by one signal synchronously, clock distribution is usually required in order to reduce the parasitic delay that is caused by asymmetrical wires [40]. During the design process, a simple transmission line model is created with $50 \Omega$ resistor, $500 \mathrm{fF}$ capacitor and $1 \mathrm{nH}$ inductor. The model is used to verify the parasitic delay results by a routing with $200 \mu \mathrm{m}$ length and $3 \mu \mathrm{m}$ width. This model generates a parasitic delay of $70 \mathrm{fs}$. Since the routing within a block is significantly smaller than this case, the delay asymmetry caused by the routing among a few gates can be ignored. However, when a large quantity of TDC blocks are combined and formed an TDC array for the multi-thread processing application [41], the clock compensate technique such as Clock Tree is absolutely necessary.

The layout view of the State Freeze module is shown in Figure 4.18. All clk pins are connected by a rail. The output pins are extracted for the convenience of connecting with the adjacent block. 

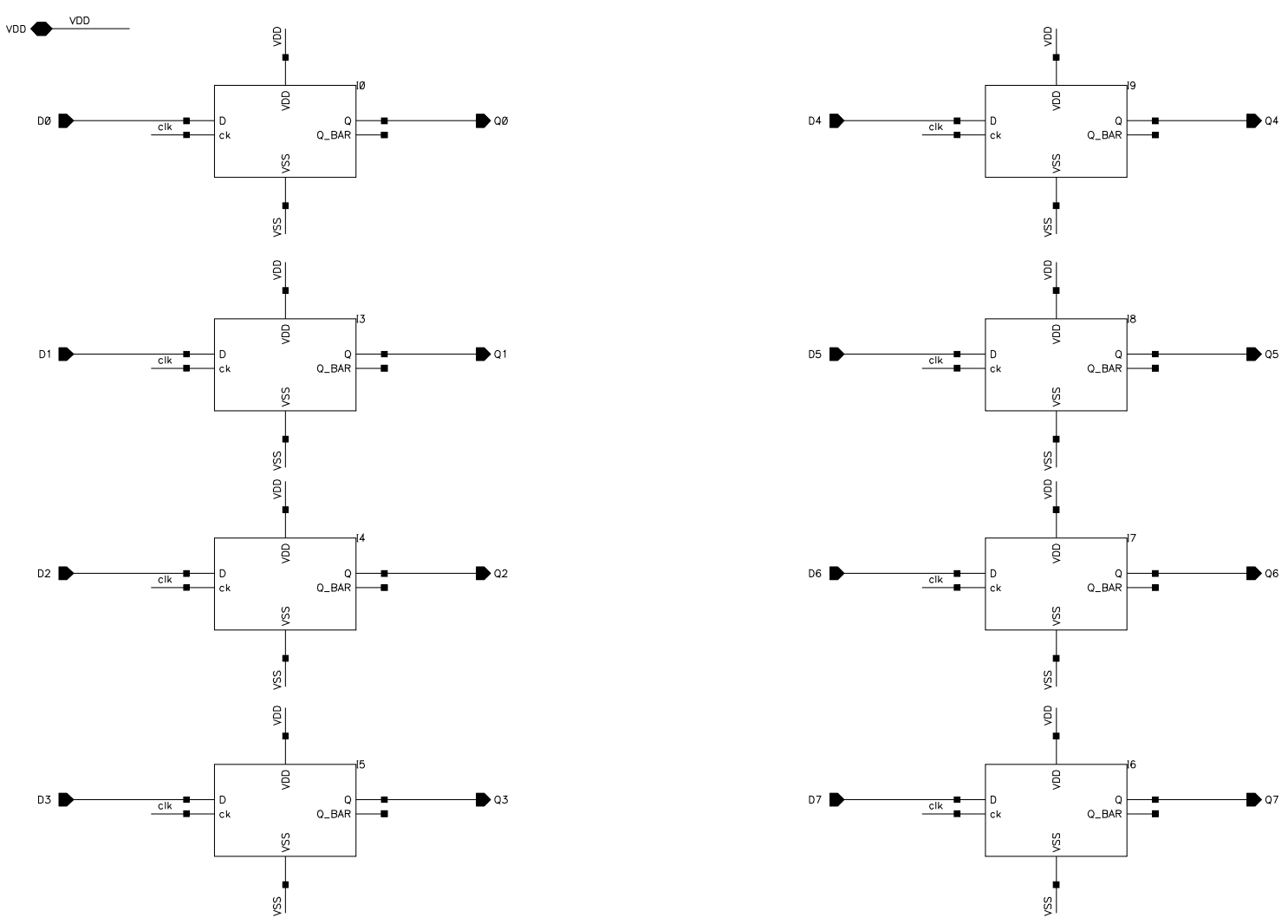

Fig. 4.17: The schematic view of the State Freeze block

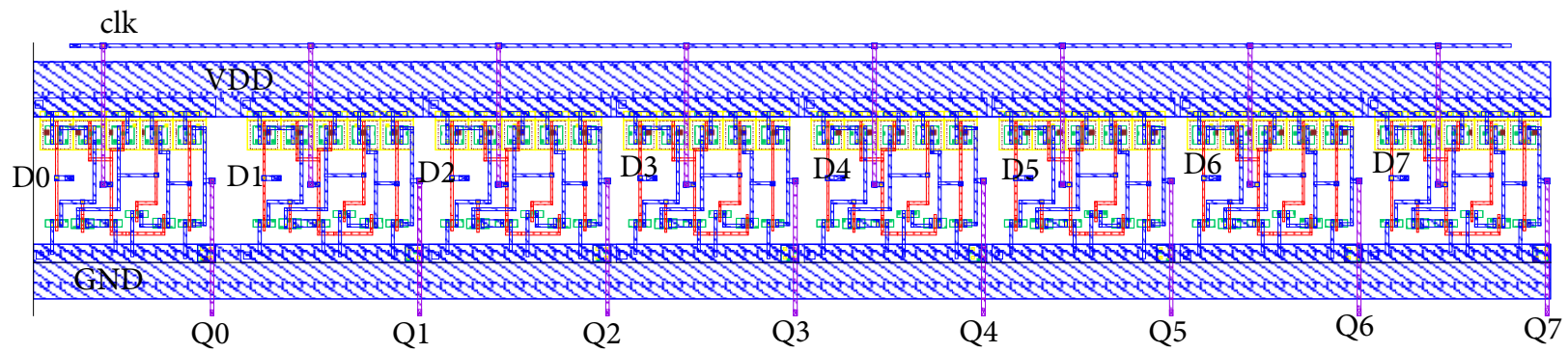

Fig. 4.18: The layout view of the State Freeze block

\subsubsection{PISO-shift Register Implementation}

With all of the components analyzed, a time domain signal has been processed and converted into a parallel binary form. The chip prototype is packaged in a CMC standard 40-pin package. Therefore, the two complementary binary outputs $N_{\text {out } 1}$ and $N_{\text {out } 2}$ which are in parallel are necessarily shifted into a serial form. This configuration 
significantly reduces the number of bond pads required. In addition, it also allows the signal to be read easily with an oscilloscope in a test environment. The last component of the switchable TDC design is the parallel-in-serial-out (PISO) shift register. The hierarchical symbol of this register is shown in Figure 4.19.

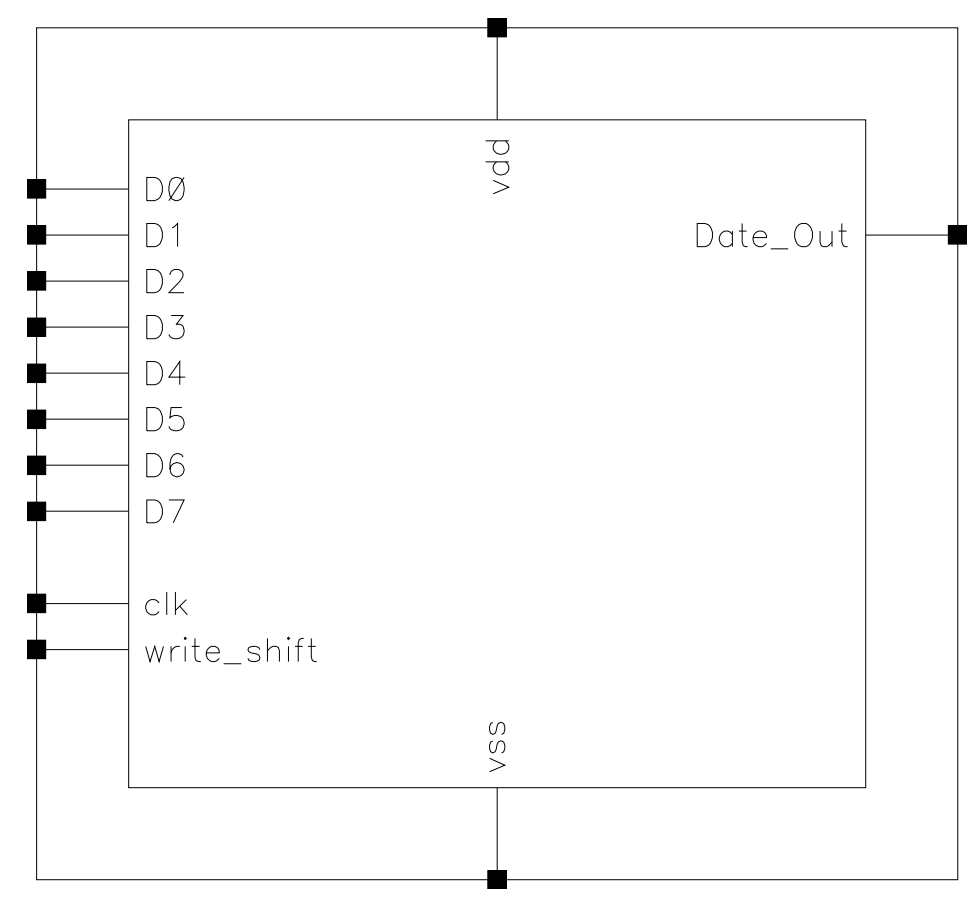

Fig. 4.19: The hierarchical symbol of the PISO shift regesiter

The specific description for each pin is shown in Table 4.8. D0 to D7 are pins where the parallel data is injected. $c l k$ is the shifting clock signal input pin. The write/shift pin is switched between 0 and 1 . For each state the circuit works in a different mode. The DateOut pin provides the converted result. How this block shifts the data will be illustrated later.

Figure 4.20 shows the schematic of the PISO shift register used by the switchable MCML-Buffer based TDC.

The PISO shift register is formed by the NAND gates and FFs discussed above. 
Table 4.8: Pins Descriptions in Figure. 4.19

\begin{tabular}{|c||c|c|}
\hline Pin Name & Type & Pin Description \\
\hline clk & I & Shifting Clock Input Pin \\
\hline Write/Shift & P & State Switching Control Pin \\
\hline D0-D7 & I & 8-bit Parallel Binary Input Pin \\
\hline DataOut & O & Serial Binary Code Output Pin \\
\hline VDD & P & 1.5V Power Supply Pin \\
\hline VSS & P & Supply Ground Pin \\
\hline
\end{tabular}

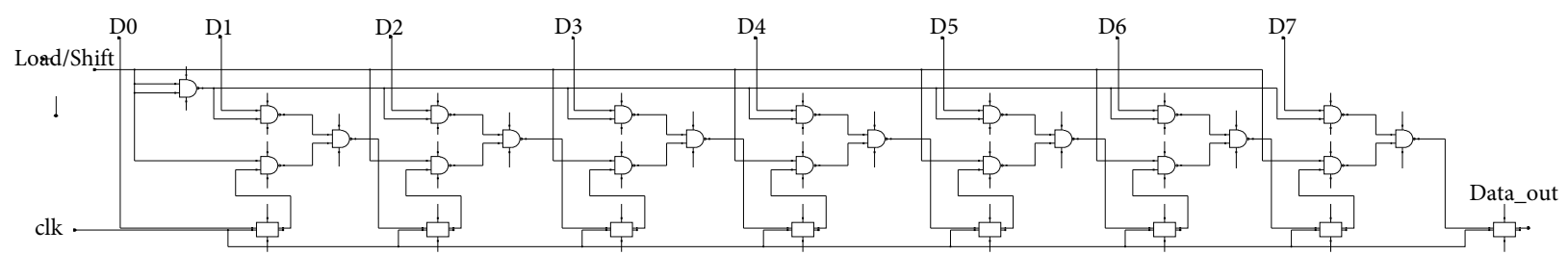

Fig. 4.20: The schematic of the PISO shift regesiter

It consists of 8 stages. Each stage consists of 3 NAND gates and $1 \mathrm{FF}$. The data shifting process is illustrated here. First the parallel data is applied to pins $D 0$ to D7 simultaneously with the Load/Shift control connected to $0 \mathrm{~V}$. After the data has been loaded, the control signal is switched to logic 1 which makes the PISO shift register work in shifting mode for the rest of the process. A periodic clock signal is injected using the $c l k$ in and the parallel data begins to be pumped out by this clock one bit after another.

The DataOut pin is loaded with a driving buffer chain. This limits the clock frequency of the pump-out clock discussed previously.

The Layout of the PISO shift register is shown in Figure 4.21. Like all components discussed before, all cells share a power supply rail and a ground rail. 


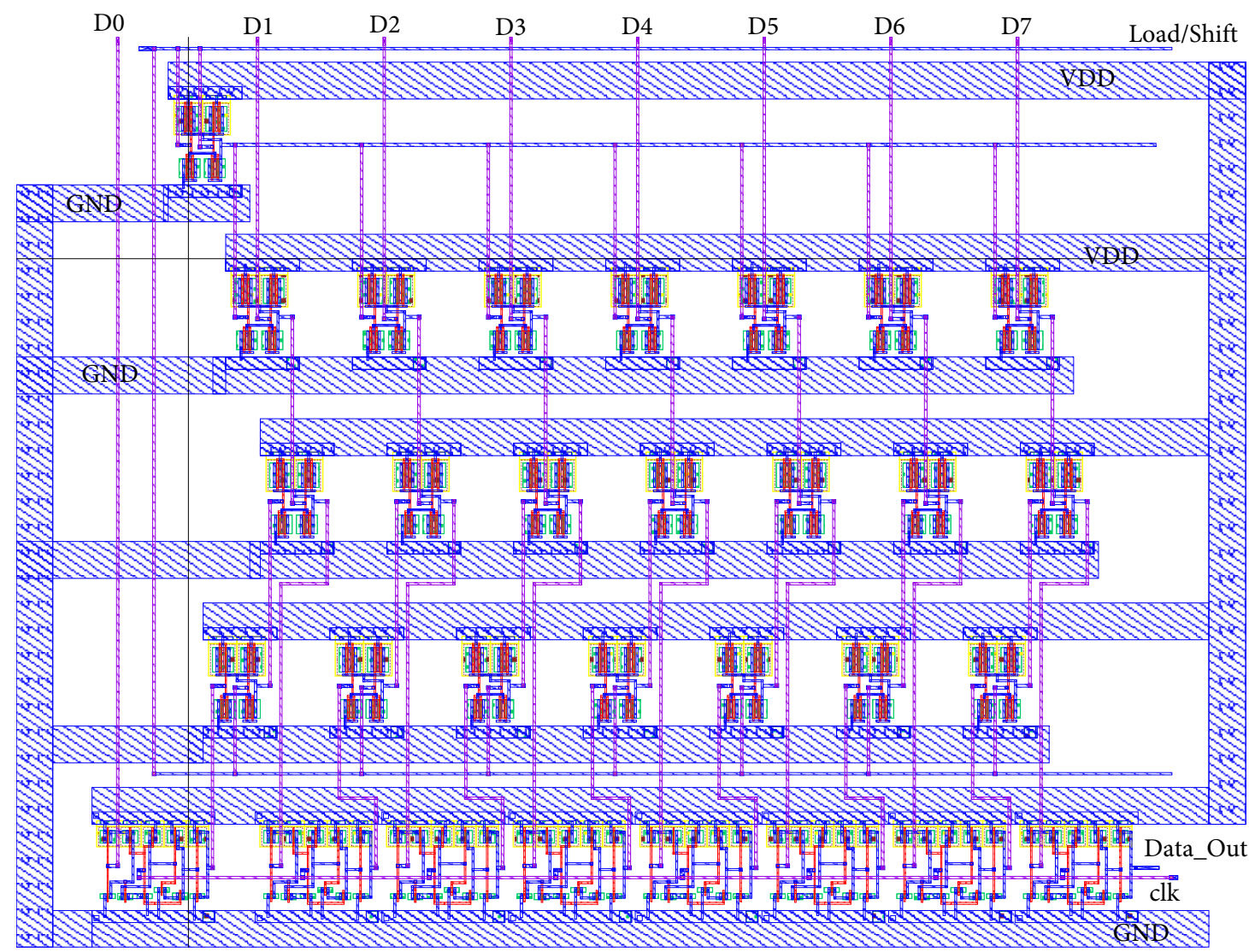

Fig. 4.21: The layout of the PISO shift regesiter

\subsection{Output Pad Driver}

The digital logic and analog circuits used to implement the Switchable MCML-Buffer based TDC are incapable of driving large capacitive loads such as bond pads and all other off chip loads. As a result, a driving buffer chain is necessarily developed. The chain is made up of classic CMOS buffers discussed previously and complete buffer chain schematic is shown in Figure 4.22.

The buffer chain contains 10 gradually enlarged stages. The 1st and 2nd stages are developed as inverters in order to reduce the dimension of the layout. Two inverters will not change the logic of the output result. The transistor sizes for each stage 


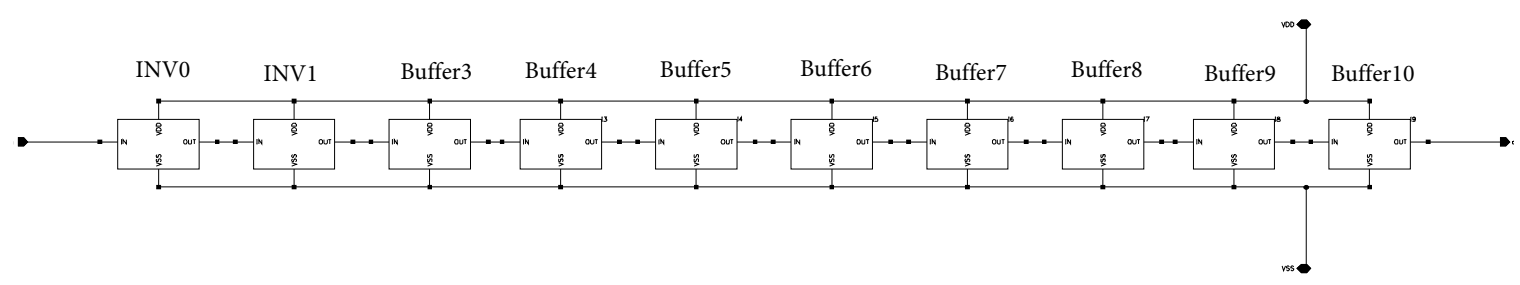

Fig. 4.22: The schematic of a driving buffer chain

are listed in Table 4.9. All transistors have the same channel length of $120 \mathrm{~nm}$ and widths shown in the table is the total width.

Table 4.9: Information of each buffer stage in the driving buffer chain

\begin{tabular}{|c||c|c|c|}
\hline Stage & Logic Type & Total width of pfet/Fingers & Width of nfet/Fingers \\
\hline INV0 & Inverter & $520 \mathrm{~nm} / 1$ & $390 \mathrm{~nm} / 1$ \\
\hline INV1 & Inverter & $520 \mathrm{~nm} / 1$ & $390 \mathrm{~nm} / 1$ \\
\hline Buffer3 & Buffer & $1.04 \mu \mathrm{m} / 1$ & $780 \mathrm{~nm} / 1$ \\
\hline Buffer4 & Buffer & $2.08 \mu \mathrm{m} / 2$ & $1.56 \mu \mathrm{m} / 2$ \\
\hline Buffer5 & Buffer & $3.12 \mu \mathrm{m} / 3$ & $2.34 \mu \mathrm{m} / 3$ \\
\hline Buffer6 & Buffer & $4.16 \mu \mathrm{m} / 4$ & $3.12 \mu \mathrm{m} / 4$ \\
\hline Buffer7 & Buffer & $5.2 \mu \mathrm{m} / 5$ & $3.9 \mu \mathrm{m} / 5$ \\
\hline Buffer8 & Buffer & $6.24 \mu \mathrm{m} / 6$ & $4.68 \mu \mathrm{m} / 6$ \\
\hline Buffer9 & Buffer & $7.28 \mu \mathrm{m} / 7$ & $5.46 \mu \mathrm{m} / 7$ \\
\hline Buffer10 & Buffer & $8.32 \mu \mathrm{m} / 8$ & $6.24 \mu \mathrm{m} / 8$ \\
\hline
\end{tabular}

The driving buffer chain is able to drive a bonding pad with $1 \mathrm{nH}$ equivalent inductance and $10 \mathrm{pF}$ equivalent capacitance. The simulation of this network is presented in the next chapter.

The layout view of the buffer chain is shown in Figure 4.23. 


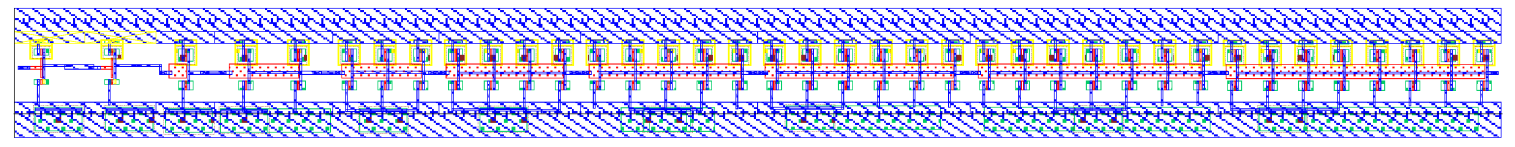

Fig. 4.23: The layout of the driving buffer chain

\subsection{Decoupling Capacitor}

A decoupling capacitor is implemented by using the ncap in the IBM CMRF8SF library. Figure 4.24 shows the layout view of the this decoupling capacitor. The capacitor is formed by the two metal layers RX and PC that are separated by an insulator. The capacitor is placed between the power supply rail and the ground rail.

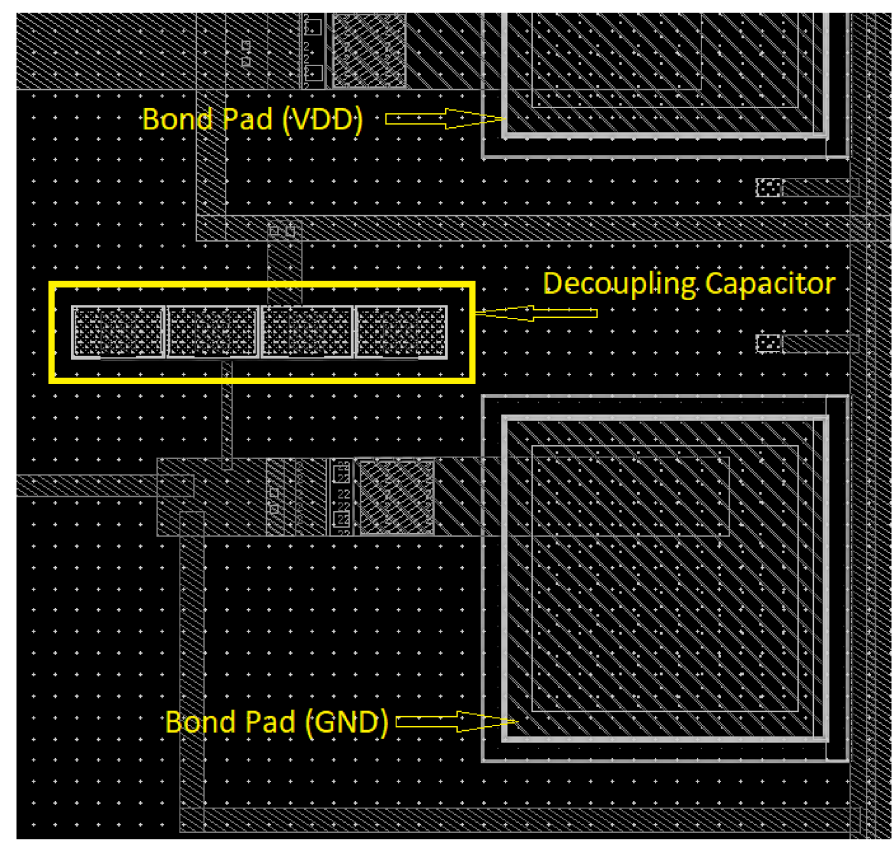

Fig. 4.24: The layout of the PISO shift regesiter

This component is made up of a ncap capacitor with multiple of 4 . For a single $n c a p$, the layout dimension is $20 \mu m \times 10 \mu m$. According to the IBM $0.13 \mu m$ CMOS technology design kits, the ncap capacitor's capacitance is $10.99 \mathrm{fF} / \mu \mathrm{m}^{2}$ where the the RX and PC layers overlap. Consequently, the total capacitance of the decoupling 
capacitor is $8.8 \mathrm{pF}$.

Even if the MCML-Buffer gates are good at suppressing the common noise due to its differential character, some of the peripheral circuits are still conventional elements and operating on a high frequency (e.g. the Loop Counter). Hence, these circuits are still sensitive to various noise sources, including switching noise, which is correlated to the switching activity and current consumption of circuits in the presence of inductive, capacitive and resistive parasitics along the power grid. The faster the circuit switch or, the more current they draw, the larger noise is seen on the supply line. If the power supply voltage drops too low, circuit functionality can be even more comprised. For example, in a $0.13 \mu \mathrm{m}$ technology, a $10 \%$ voltage variation bas been shown to result in a $30 \%$ variation in the delay of a gate [42]. Thus, a decoupling capacitor is necessary. 


\subsection{ESD Protection}

An electrostatic discharge (ESD) protection device is provided by the IBM CMRF8SF library and used in this design to protect the circuit. The ESD protection device consists of two diodes. The anode of the first diode is connected to the bond pad and the cathode of this diode is connected with the positive power supply. The protection is achieved by forward biasing this diode in order that the ESD current flows into the positive power supply instead of into the transistors. The second diode's cathode is linked to the bond pad and its anode is connected to ground. As with the first diode, large ESD events are prevented by forward biasing the second diode so that the ESD current is forced to flow into the ground.

Figure 4.25 shows the schematic of a single ESD protection device and its corresponding layout view. 


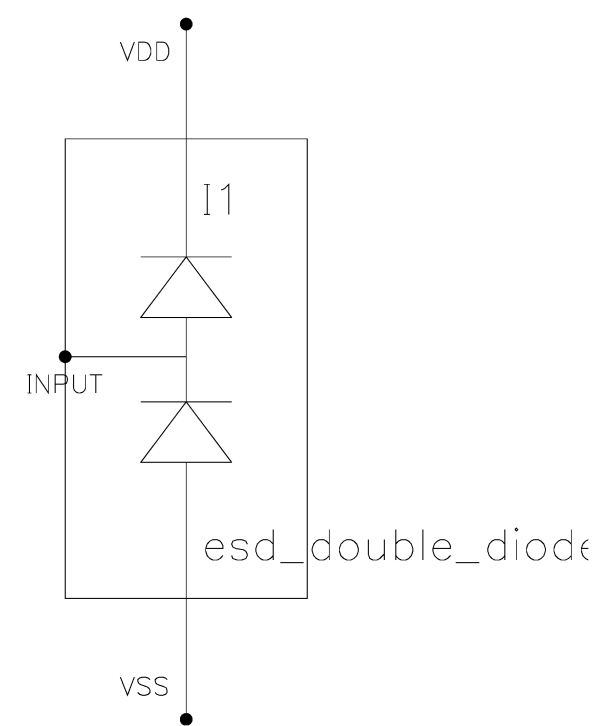

(a) schematic

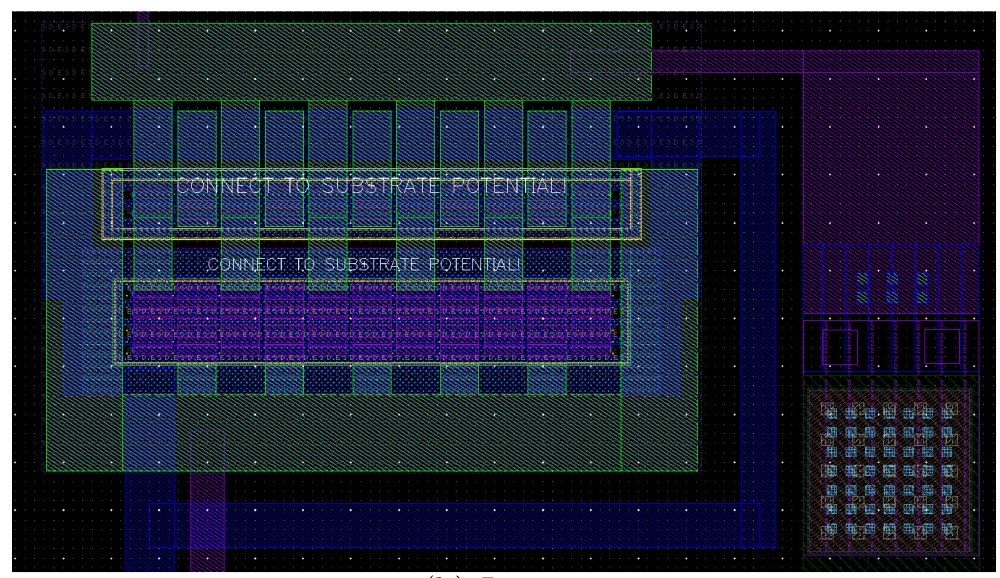

(b) Layout

Fig. 4.25: Schematic of the ESD protection device and the corresponding layout

\subsection{Chip Micrograph}

The unpackaged die was probed using a probe station. The micrograph is shown in Figure 4.26. Due to the fact that all components are covered under the top metal layer (Metal 8 in this CMOS technology), it is impossible to identify all components from this top view micrograph. Hence, the location of each block has been annotated 
on this photo.

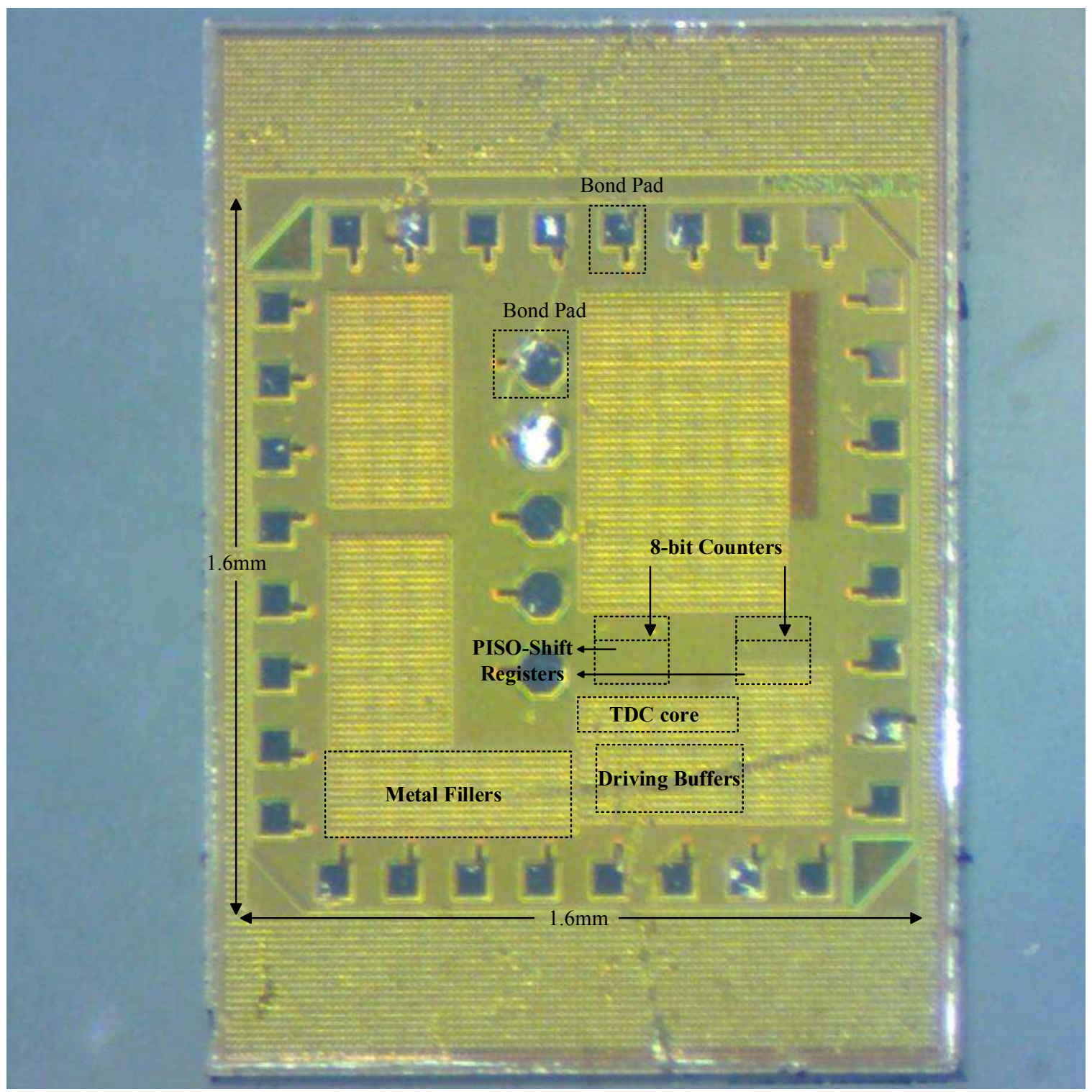

Fig. 4.26: The micrograph of the chip. Main components are highlighted. 


\subsection{Chip Layout}

The top layout view of the 4-Step Switching MCML-Buffer based TDC is shown in Figure 4.27. It should be mentioned that the portion highlights with dash line is irrelevant to this work. In order to fully use the chip area, a member from my supervisor's group placed his design here. 


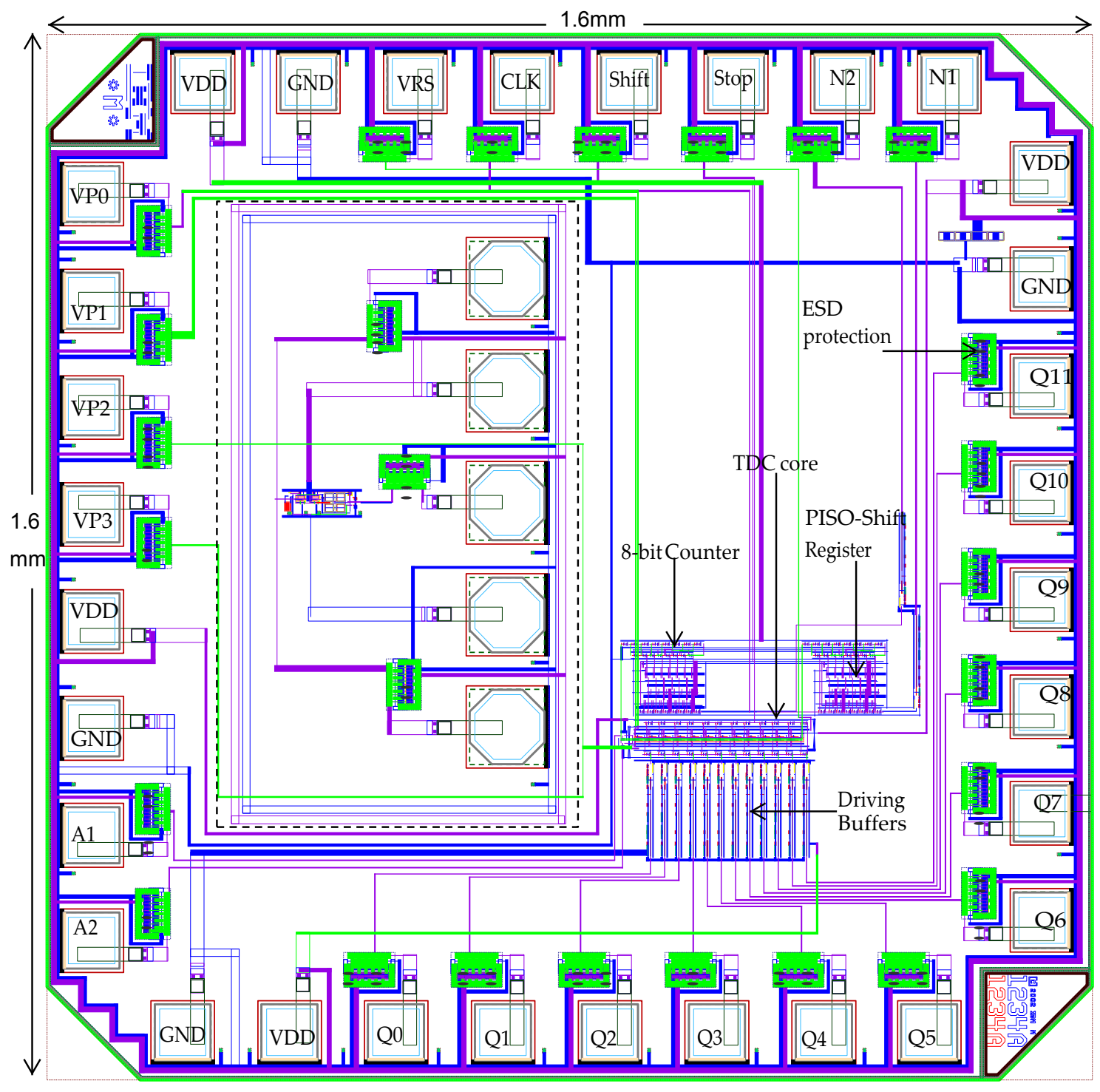

Fig. 4.27: Top view of the switchable MCML-Buffer based TDC chip layout. The major building blocks of the novel TDC are a TDC core, counters, PISO-shift registers and driving buffers. The chip margin is $1.6 \mathrm{~mm}$ tall and $1.6 \mathrm{~mm}$ wide. 


\section{Chapter 5}

\section{Switchable MCML-Buffer based TDC Simulation Results}

\subsection{Introduction}

This chapter introduces the simulation methodologies and results of the Switchable MCML-Buffer based TDC. The simulation that is based on the layout view is not accurate. In order to address this issue, all blocks were extracted into extracted view. This process (default RC extraction) generates the parasitic resistance and capacitance in the extracted view. Therefore, the post-layout simulation are more precise than the layout simulation. All simulation results are based on the extracted view in this chapter.

\subsection{Delay Cell Simulated Performance}

\subsubsection{Switchable MCML-Buffer Step Response}

A test bench was created in order to measure the delay of a fundamental delay cell, which is a switchable MCML-Buffer gate in this work. The 4 steps buffer gate was 
functionally verified via a transient analysis performed using the Cadence Spectre simulator in the Analog Design Environment (ADE). The simulation is performed with the TT (typical-typical) corner with the nominal temperature of $27{ }^{\circ} \mathrm{C}$. The total simulation time is set to be 10 ns. Two signal generator (Vpulse) generate a pair of complementary clock signals with $180^{\circ}$ phase difference. The trigger event was then applied to the input pins of the first stage and enabled the signal transmission. The test bench is shown in Figure 5.1.

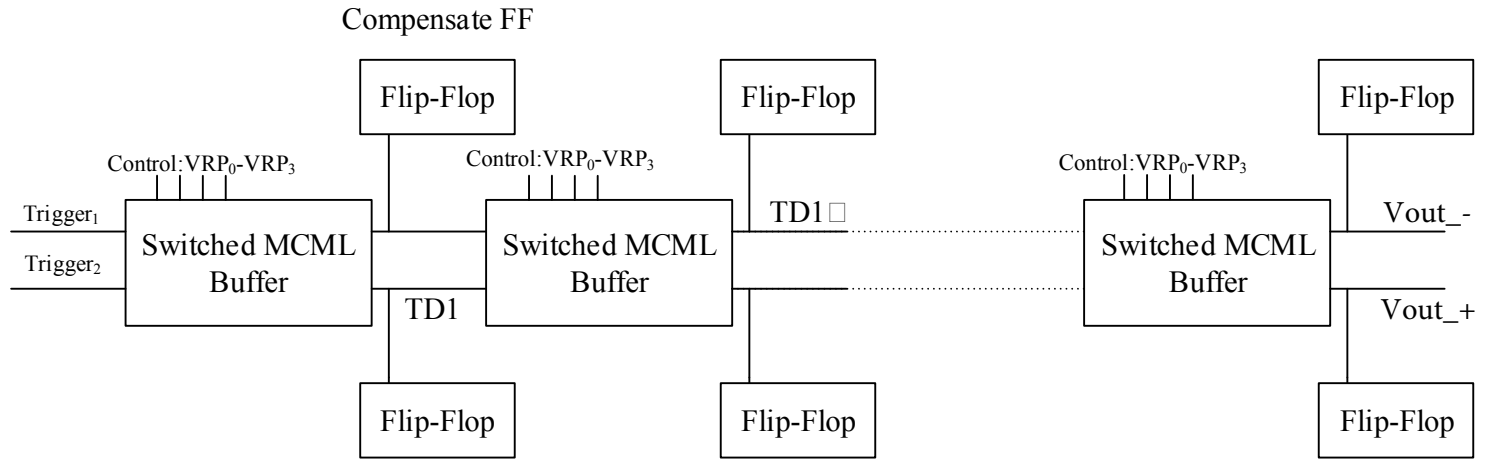

Fig. 5.1: The test bench for the measurement of specific delay for each switched condition. With different control voltage combination (VRP0-VRP3). The MCML-Buffer delay element operates with different propagation delays.

As Figure 5.1 shows, compensation FFs are loaded by each stage in order to probe the propagation of the signal with correct load capacitance. Even though the input capacitance $C$ in of the Flip-Flop is relatively small compared with the $C$ in of a MCML-Buffer gate, it still involved capacitance and degraded the signal's propagation time. For the leading signal trigger1, its copy, which was delayed by a delay stage is $T D 1$. Obviously, the delay cell step response varies with switched conditions. The cell propagation delay is defined by the $50 \%$ difference in the two signals.

A transient analysis is used to verify the specific step response of all four of the different operation conditions. The simulation result is shown in Figure 5.2. For each 
curve, a control voltage is applied to the delay cells to tune the response.

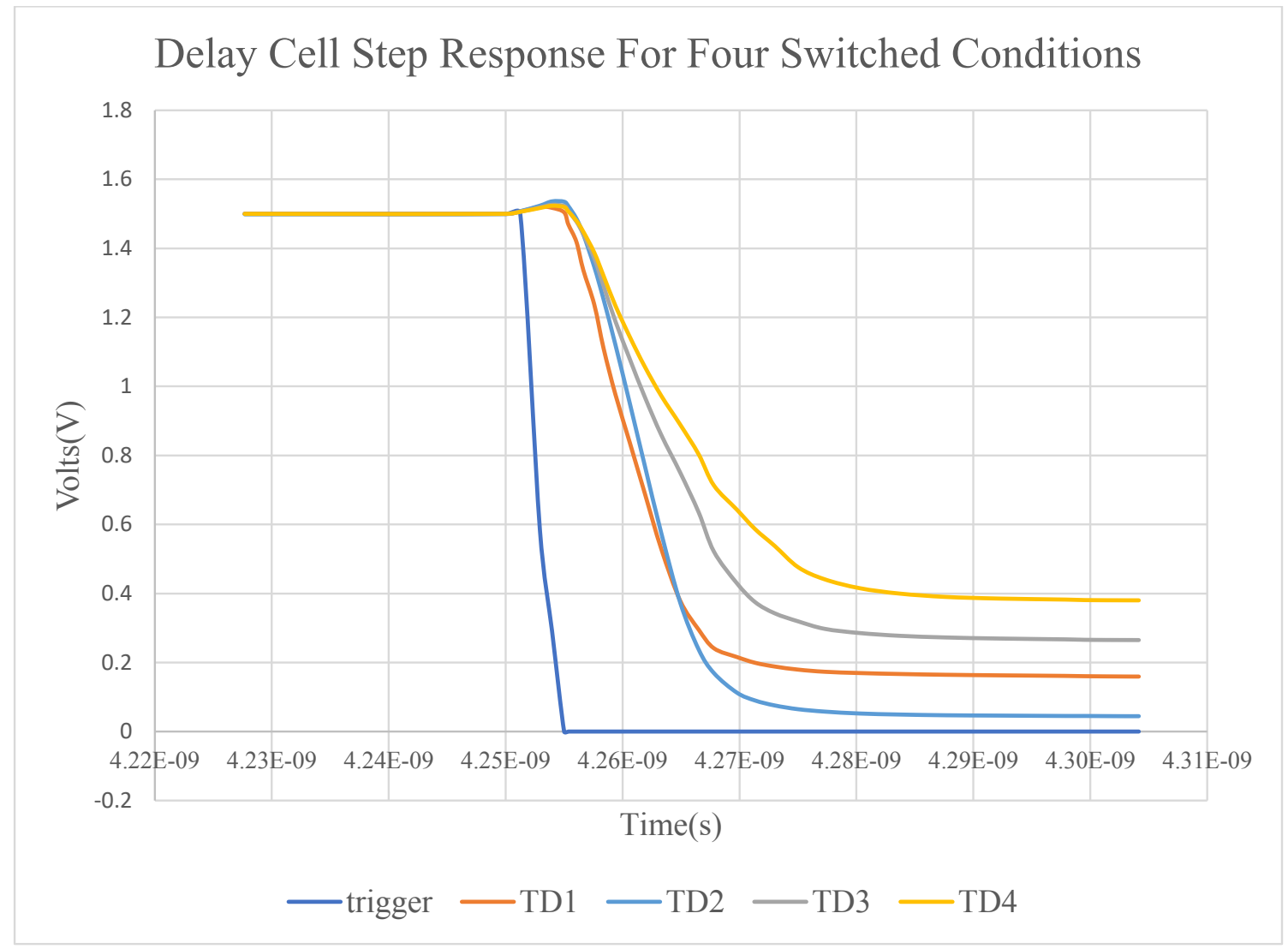

Fig. 5.2: Delay cell step response for the four switched conditions, with time delay: $\mathrm{TD} 1=8.24 \mathrm{ps}, \mathrm{TD} 2=10.83 \mathrm{ps}, \mathrm{TD} 3=12.98 \mathrm{ps}$, and $\mathrm{TD} 4=14.3 \mathrm{ps}$

As shown in Figure 5.2, the minimum step response $T D 1$, namely the resolution $T_{L S B}$, is $8.24 \mathrm{ps}$. This mode is achieved by enabling all four of the active loads of each delay cell.

The second operation mode is achieved by enabling 3 active loads. In this situation, three control signals should be applied to a $0 \mathrm{~V}$ power source. When the TDC operates on this mode, the resolution is equal to the time delay TD2 (10.83 ps).

As in the last mode, the third operation point requires 2 enabled loads. The exact resolution of this mode is equal to TD3 (12.98 ps).

For the fourth operation mode, only one active load is involved. This configuration 
achieves the lowest resolution, which is equal to TD4 (14.3 ps). 


\subsubsection{Corner Simulation of the Delay Cell}

Multi-corner simulations are performed in order to verify the variance of specific step response. Three temperature values include: $-40,0$ and $125^{\circ} \mathrm{C}$ are chosen to observe the variations caused by different temperature environments. Furthermore, two process corners of FF and SS are set. The FF corner presents low PMOS/NMOS threshold voltage situation and the SS corner represents the converse situation. In all, 6 corners are simulated by using the ADE XL multi-corner simulator. The results of these 6 corners and the nominal step responses are shown in Table 5.1.

Table 5.1: Multi-corner analyses results

\begin{tabular}{|c|c|c|c|c|}
\hline Corner & Mode1 ps & Mode2 ps & Mode3 ps & Mode4 ps \\
\hline$-40\left({ }^{\circ} \mathrm{C}\right)(\mathrm{FF})$ & 5.31 & 7.08 & 9.29 & 10.64 \\
\hline $0\left({ }^{\circ} \mathrm{C}\right)(\mathrm{FF})$ & 5.91 & 7.77 & 10.84 & 11.37 \\
\hline $125\left({ }^{\circ} \mathrm{C}\right)(\mathrm{FF})$ & 7.69 & 9.69 & 12.51 & 13.44 \\
\hline $27\left({ }^{\circ} \mathrm{C}\right)(\mathrm{TT})$ & 8.24 & 10.83 & 12.98 & 14.3 \\
\hline$-40\left({ }^{\circ} \mathrm{C}\right)(\mathrm{SS})$ & 8.75 & 11.9 & 12.16 & 16.01 \\
\hline $0\left({ }^{\circ} \mathrm{C}\right)(\mathrm{SS})$ & 9.8 & 13.15 & 13.49 & 17.2 \\
\hline $125\left({ }^{\circ} \mathrm{C}\right)(\mathrm{SS})$ & 12.9 & 16.54 & 18.6 & 20.17 \\
\hline
\end{tabular}

This table shows all possible step responses with the environment variation. The best case is $5.31 \mathrm{ps}$ delay because the circuit is simulated with $-40{ }^{\circ} \mathrm{C}$ temperature corner and the FF model group. The worst case is 20.17 ps cell delay, due to its corresponding $125{ }^{\circ} \mathrm{C}$ corner and SS process model.

Two charts are created in order to intuitively illustrate the corner simulation results. Figure 5.3 shows all temperature corners $(-40,0,125)$ with FF process model group and the nominal corner result.

Simulation results of all temperature corners with SS model group are combined 


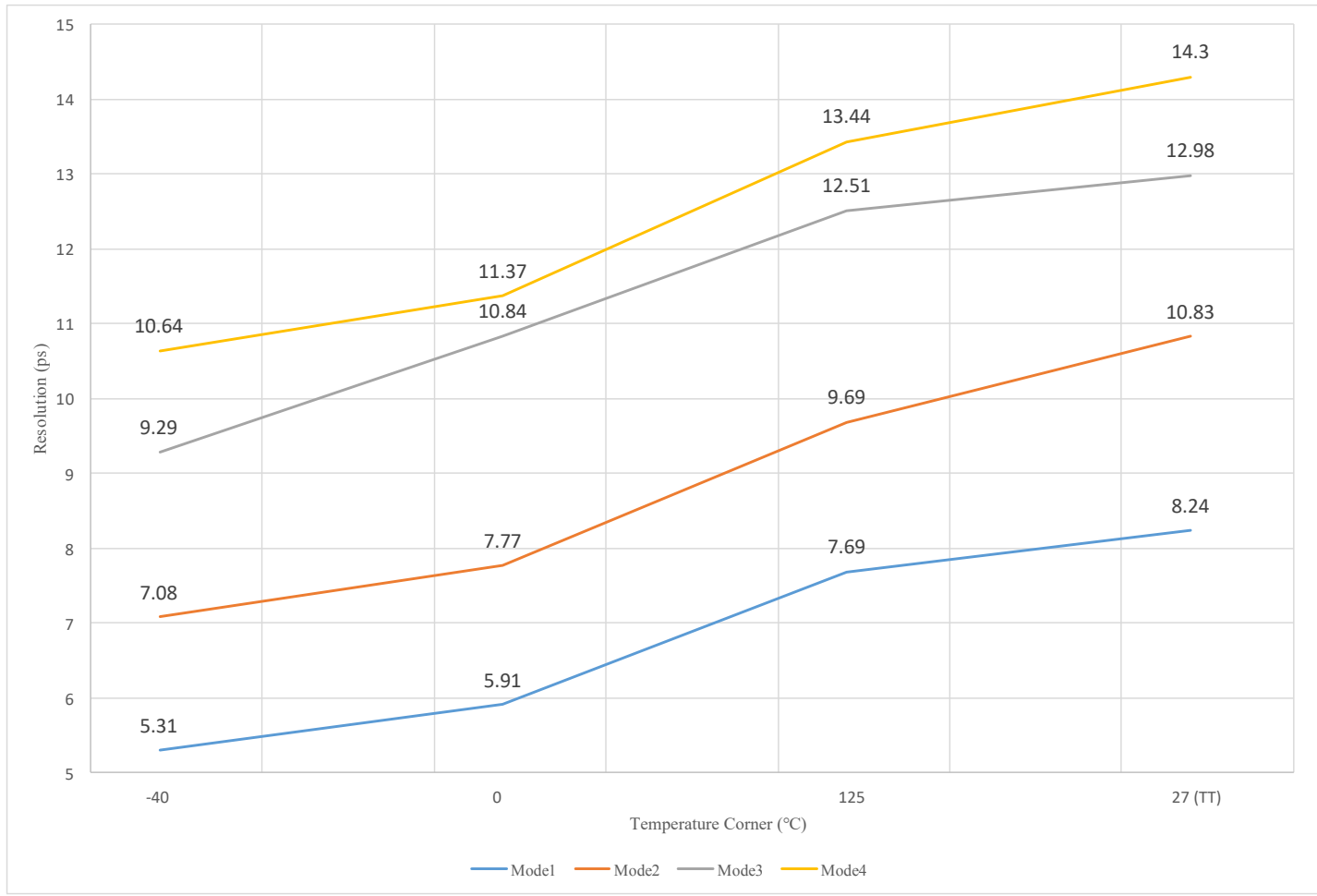

Fig. 5.3: Step response variations for different temperatures with FF corner simulation.

with the nominal step response in Figure 5.4.

According to the simulation results, the process and temperature variation are either able to enhance the time domain delay by approximately $20-35 \%$ or degrade it by $40-55 \%$. This is due to high temperature implies more thermal noise and random collisions of electrons, thus device resistance increases and electron mobility decreases. In another aspect, the FF corner assumes PMOS/NMOS have lower threshold voltage compare to TT corner. Low threshold voltage devices switch faster and lead to the smaller step response in FF corner simulation. 


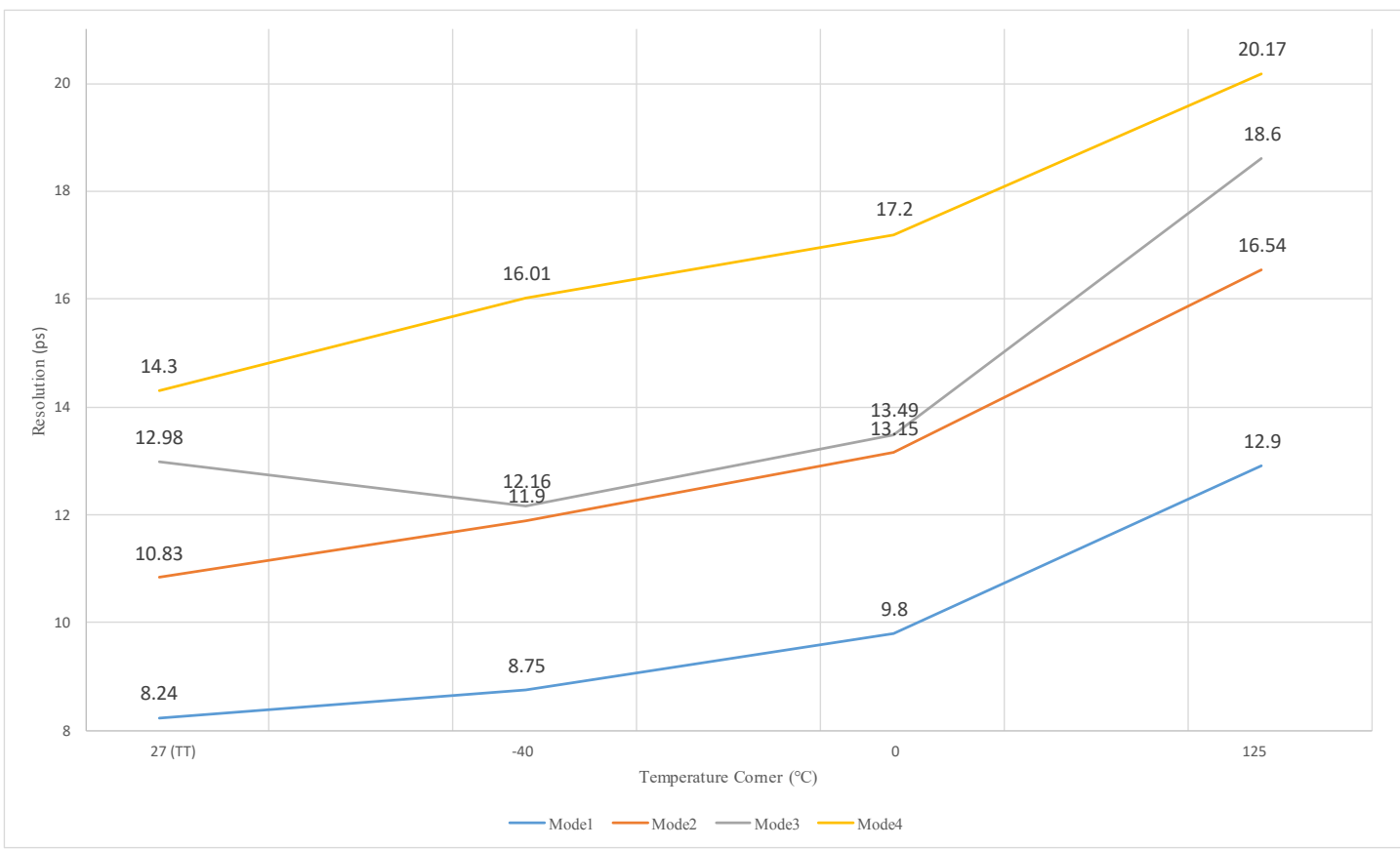

Fig. 5.4: Step response variations for different temperatures with SS corner simulation.

\subsection{TDC Core Simulated Performance}

\subsubsection{Calculation of the Loop Delay}

The TDC designed in this work is able to work with four modes. Hence the rising

time $t_{p L H}$ and the falling time $t_{p H L}$ for the propagation signal is unable to be fully symmetric for all of the four different cases. Figure 5.5 shows an example of this phenomenon. The time interval of one differential output (e.g. blue curve labeled as $D 1$ ) is unequal with the its corresponding differential signal (e.g. orange curve labeled as D2). Fortunately, the differential character of TDC core is capable of addressing this asymmetry inherently by counting both the upper and lower delay path. 
As shown in Figure 5.5, the test bench involves an equation

$$
t_{\text {Loop }}=D 1+D 2
$$

where $t_{\text {Loop }}$ is the equalized value, which gives the definition of the loop cycle. D1 and D2 correspond to a pair of complementary half-cycle in the differential delay loop. This equation is used to define the loop delay of the TDC core. By involving this equation into the test bench, the simulator would be able to calculate the exact time length of the loop delay and output its value with each run of the simulation. This process significantly enhances the efficiency of the circuit optimization.

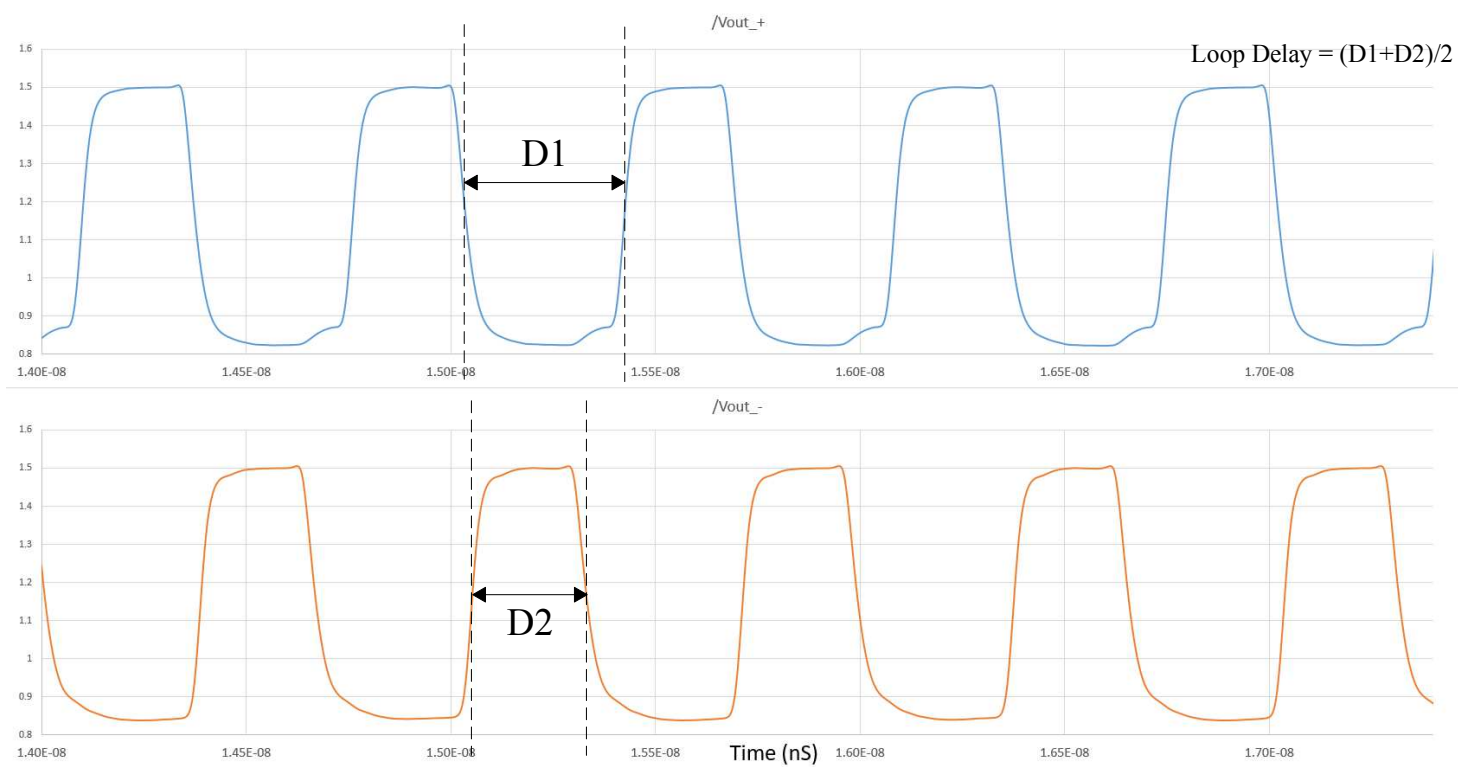

Fig. 5.5: An average value of the loop delay for the complementary path is equalized. This configuration is able to address the delay asymmetry that is shown in this figure.

In the next subsection, the TDC core will be simulated with four different modes. The parameters include: the resolution, loop delay, number of cycles, thermometer code output. The power consumption will be presented separately for each mode. 


\subsubsection{The TDC Performance with 4 Loads Activated}

A test bench was built for the purpose of measuring the performance of the TDC core. It previously stated that the Switchable MCML-Buffer based TDC designed in this work is able to operate with four different resolutions. For the TDC core block, each operation mode is simulated with a $25 \mathrm{~ns}$ transient analysis. With all the reference voltage from $V P 0$ to $V P 3$ connected to $0 \mathrm{~V}, 4$ active loads are enabled and the TDC operates on the minimum resolution which is $8.24 \mathrm{ps}$.

The start event begins from $1 \mathrm{~ns}$ and the stop signal is triggered on the $21 \mathrm{~ns}$. Consequently, the time interval to be measured is equal to $20 \mathrm{~ns}$. This TDC is designed for the specific application (working as the TOF converting element in LIDAR system). Thus, twenty nanosecond is a meaningful time interval since it represents $10 m-100 m$ level distance detection.

Figure 5.6 shows the waveforms of the outputs Vout1 and Vout2 when the TDC works in this mode.

The simulation result shows that the first loop counter that is in charge of counting the upper delay path, gives out a record of 51 full cycles. The lower signal propagates 50 cycles before the stop event, resulting in the total number of loop cycles in this case to be equal to $N_{1}+N_{2}$ which is 101 . The simulation result also provides the loop delay $T_{\text {loop }}$ for this case, which is equal to $197.76 \mathrm{ps}$. The coarse counting result is equal to $\left(N_{1}+N_{2}\right) T_{\text {loop }}=19973.76 \mathrm{ps}$.

The 12-bit thermometer output result is shown in Figure 5.7. At the rising edge of the stop signal, the one-zero transition appears between the 4 th and 5 th stage. It means that the start signal has passed 4 stages. Therefore, the fine counting result is $4 \times T_{L S B}=32.96 p s$.

The total of the coarse and fine measurement result is $20006.72 \mathrm{ps}$. When the 


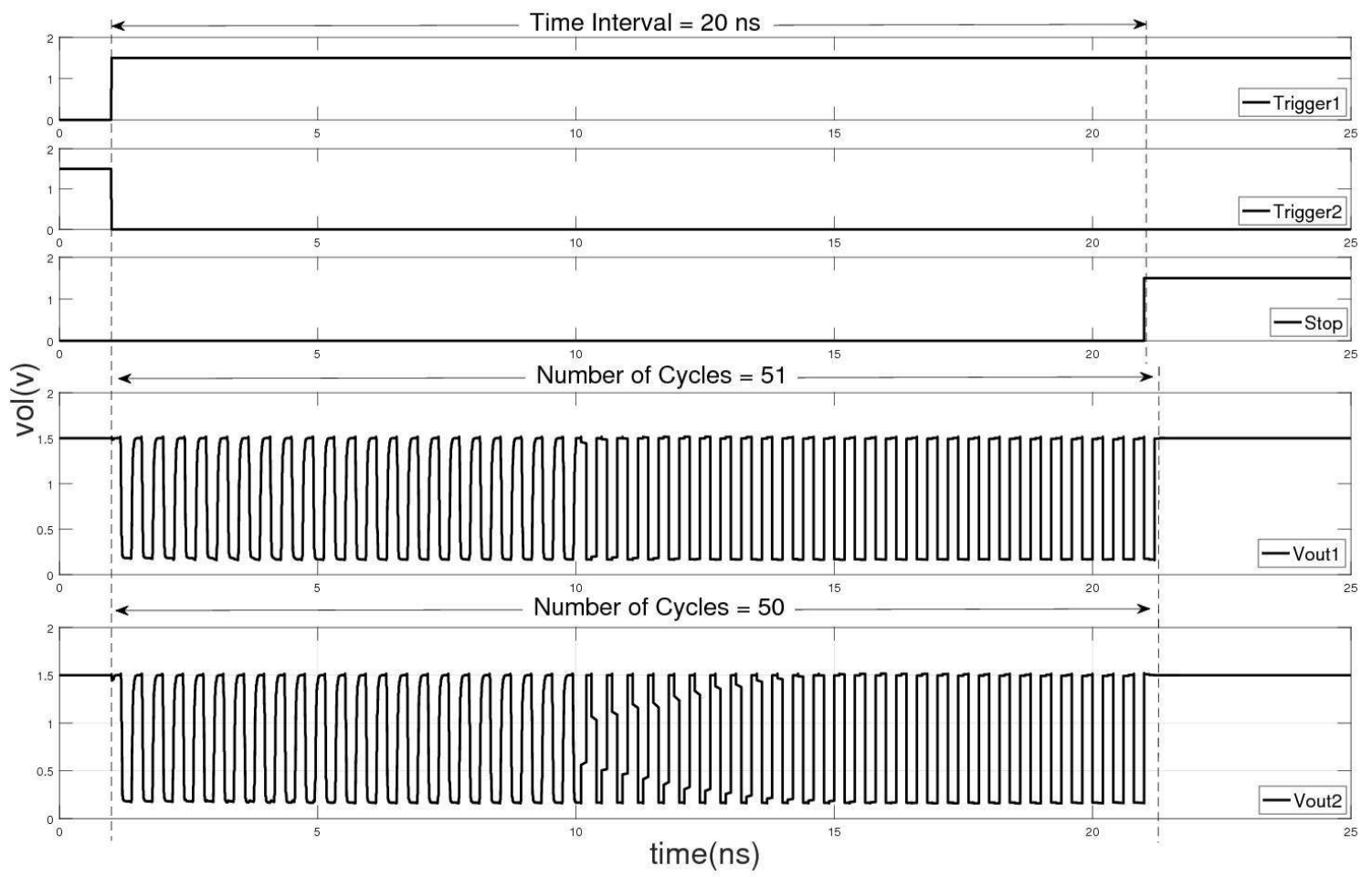

Fig. 5.6: The Simulation Result of a $20 \mathrm{~ns}$ Time Interval. The total number of cycles of the upper path is 51 and the lower path is 50 .

TDC operates on this mode (resolution $=8.24 \mathrm{ps}$ ), the error of a single conversion is 6.72 ps.

The test bench also provides the average power consumption of this operation point. The power of the TDC core block consists of two components. One is the MCML-Buffer loop power consumption which is constant. The other component is the power of the FFs and the Ring Closer block. The power of these devices is dynamic. Consequently, the simulation result of the average power is $19.29 \mathrm{~mW}$ 


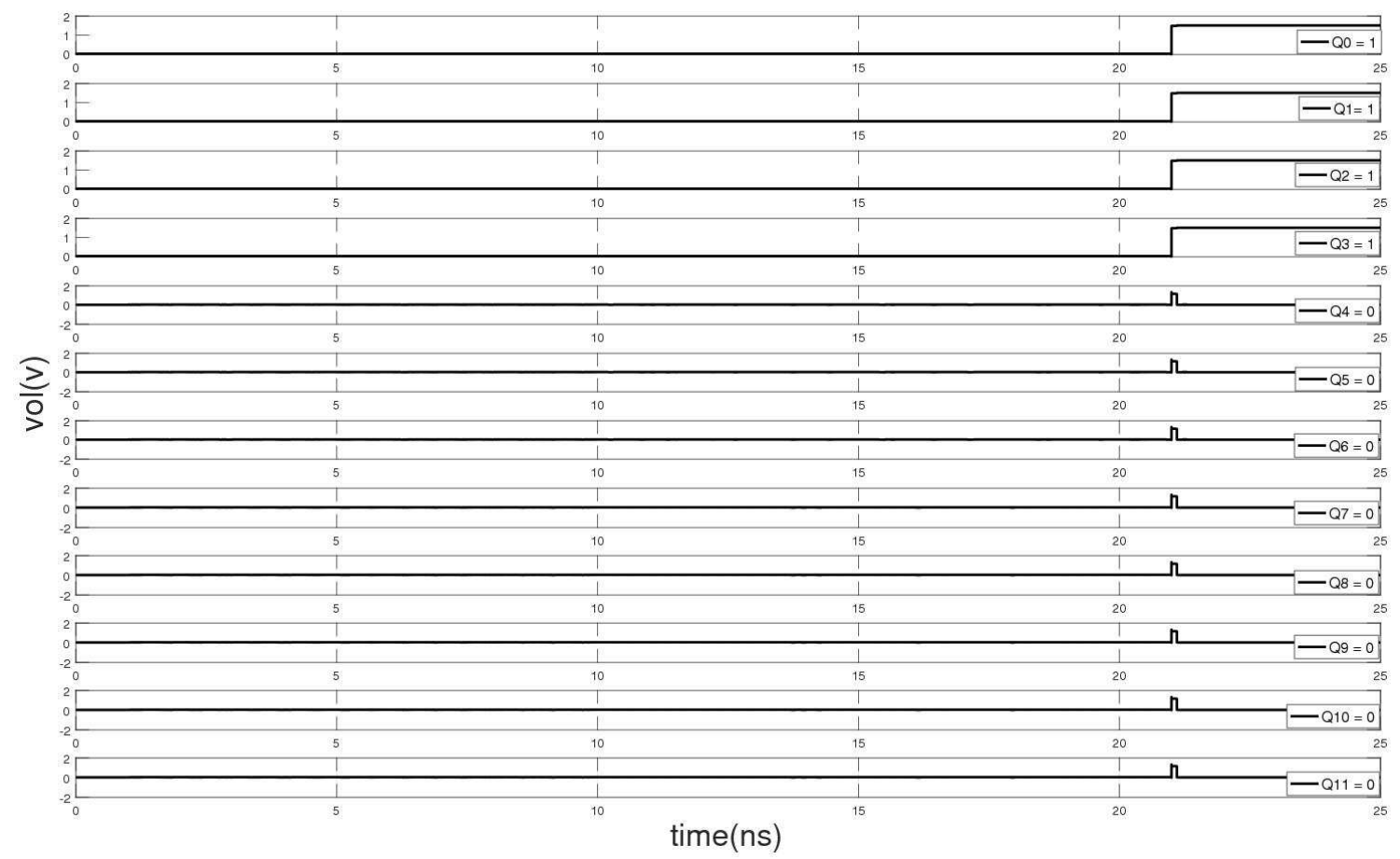

Fig. 5.7: The simulation results of the FFs. A 12-bit thermometer code is acquired. It contains 4 bits of logic 1 followed by 8 bits of logic 0 .

\subsubsection{The TDC Performance with 3 Loads Activated}

The control voltages $V P 0, V P 1$ and $V P 2$ are connected to $0 \mathrm{~V}$ in this simulation. The TDC operates on the second mode with this configuration which means the resolution is equal to $10.83 \mathrm{ps}$. The same as above, start event begins from $1 \mathrm{~ns}$ and the stop signal is triggered on the $21 \mathrm{~ns}$. Figure 5.8 shows the waveforms of the outputs Vout1 and Vout 2 when the TDC works is in this mode.

The simulation result shows that the upper delay path oscillates for 35 cycles during the reference time interval. The lower signal propagates for 36 cycles before the stop event. Therefore, the total number of loop cycles in this case is equal to $N_{1}$ $+N_{2}$ which is 71 . The simulation result also provides the loop delay $T_{\text {loop }}$ for this case, which is equal to $281.75 \mathrm{ps}$. The coarse counting result is equal to $\left(N_{1}+N_{2}\right) T_{\text {loop }}$ 


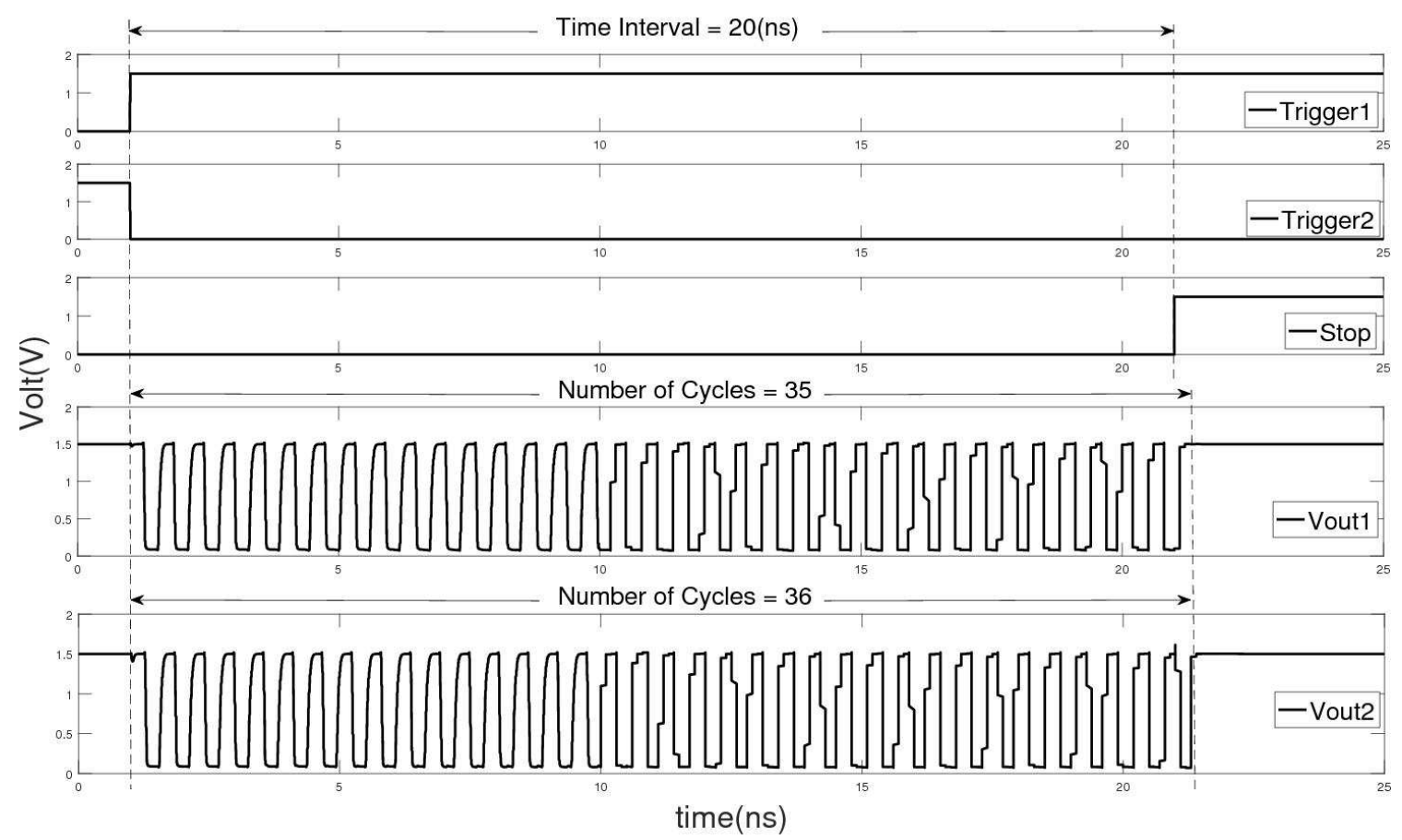

Fig. 5.8: The Simulation Result of a 20 ss Time Interval. The total number of cycles for the upper path is equal to 35 and the lower path is 36 .

$=20004.25$ ps .

The 12-bit thermometer output result is shown in Figure 5.9. At the rising edge of the stop signal, the one-zero transition appears between the 9th and 10th stage. Confirming the start signal has passed 9 stages. Therefore, the fine counting result is $9 \times T_{L S B}=97.47 \mathrm{ps}$.

The total of the coarse and fine measurement result is 20101.72 ps. The error of a single conversion is equal to $101.72 \mathrm{ps}$ in this case. The simulation result of the average power when the TDC operates on this mode is $15.26 \mathrm{~mW}$. The power is lower than the 1st mode due to the lower resolution. 


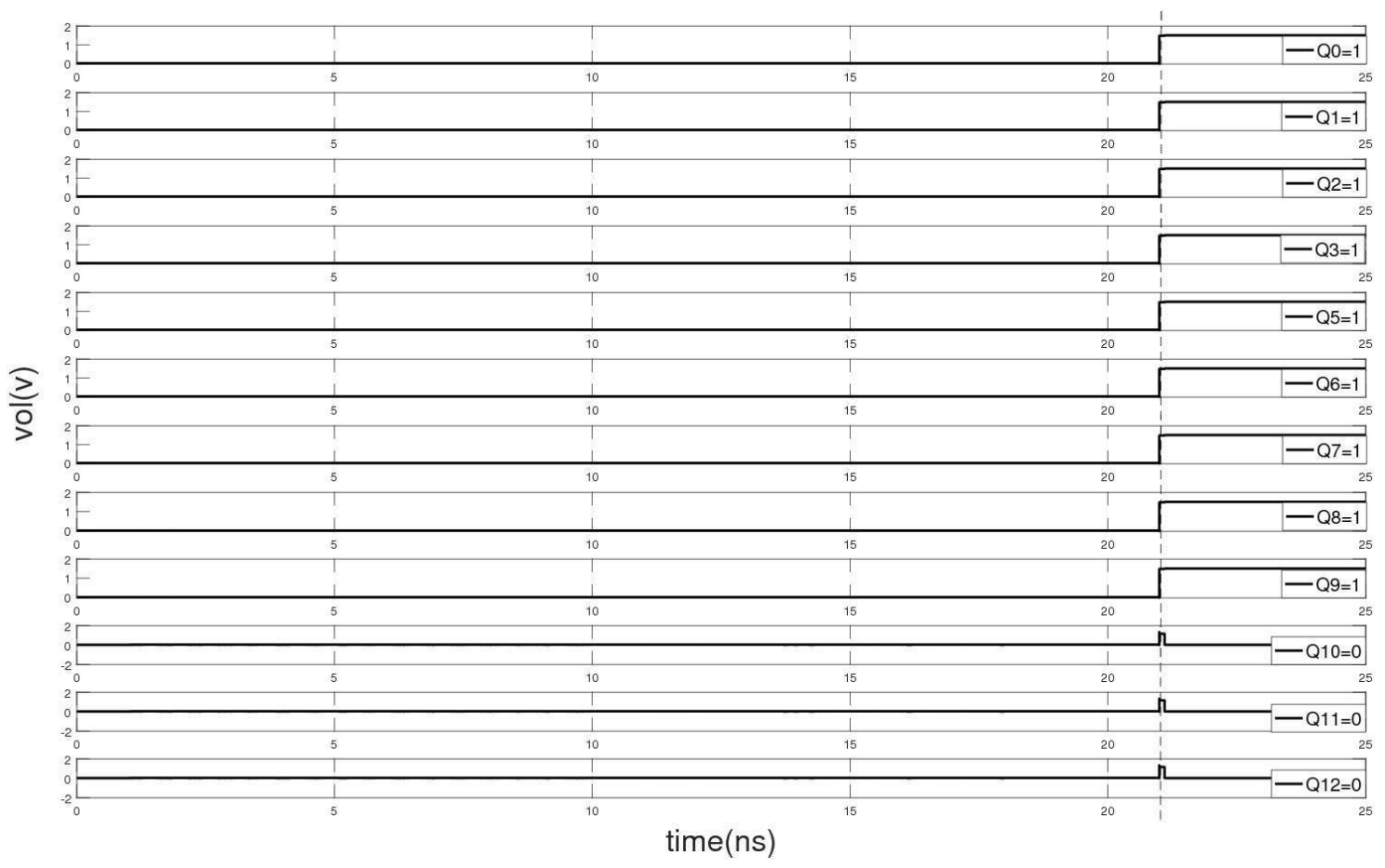

Fig. 5.9: The simulation results of the FFs. A 12-bit thermometer code is acquired. It contains 9 bits of logic 1 followed by 3 bits of logic 0 .

\subsubsection{The TDC Performance with 2 Loads Activated}

Both the control voltages $V P 0$ and $V P 1$ are switched to $0 \mathrm{~V}$ to set up the third operation point of the TDC. In this case the resolution is $12.98 \mathrm{ps}$. The same transient analysis is used to simulate the performance of the TDC core block. Figure 5.10 shows the waveforms of the outputs Vout1 and Vout 2 when the TDC works in this mode. The reference time interval is still represented by the time difference between the start and stop signal.

There is no doubt that the total loop cycles will be less than the previous condition since the resolution of this mode is degraded. The simulation result matched this conclusion. It demonstrates the upper delay path oscillates for 30 cycles during the reference time interval. The lower signal propagates for 29 full cycles before the 


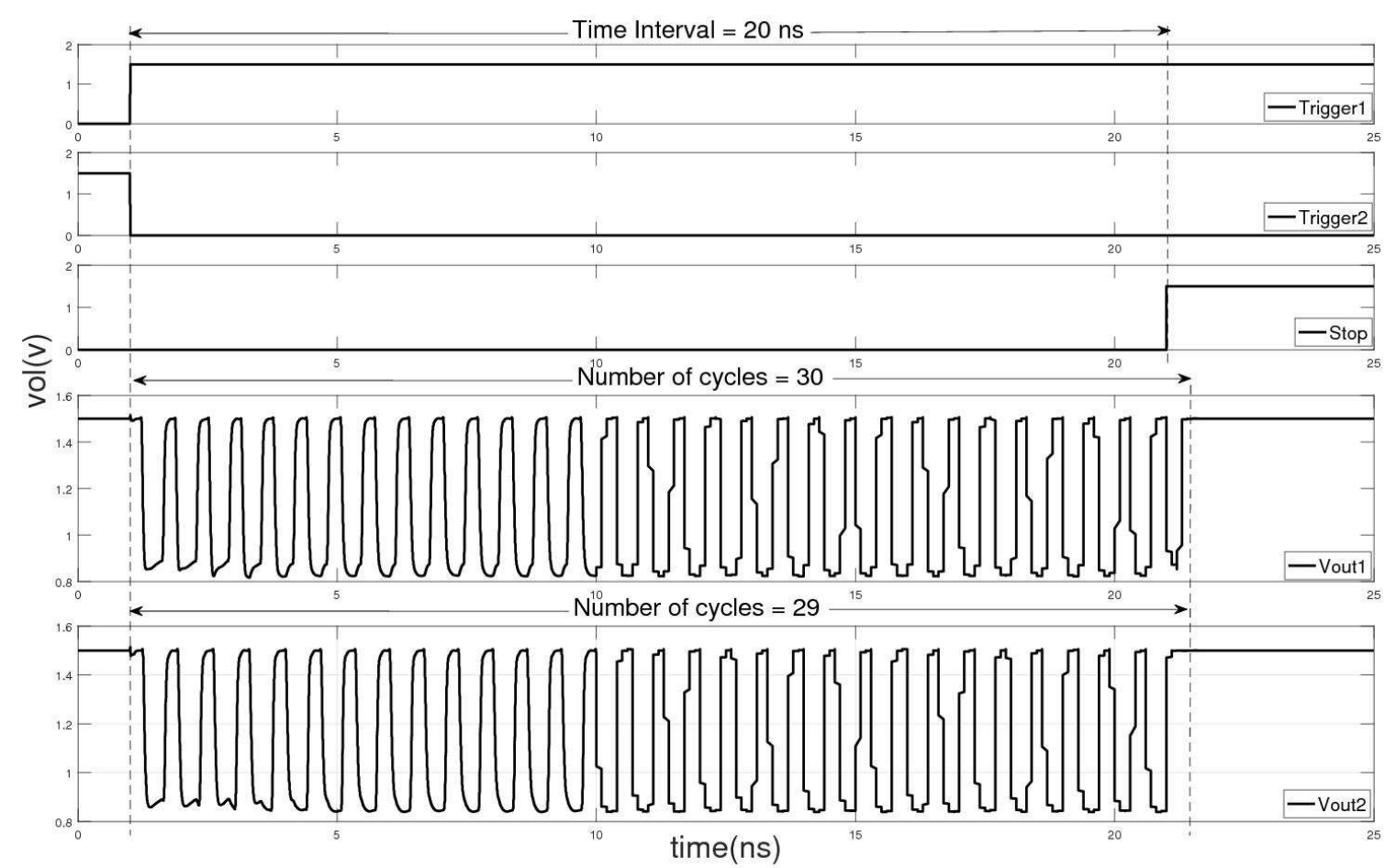

Fig. 5.10: The Simulation Result of a $20 \mathrm{~ns}$ Time Interval. The total number of cycles for the upper path is equal to 30 and the lower path is 29 .

stop event. Therefore, the total number of loop cycles in this case is equal to $N_{1}+$ $N_{2}$ which is 59 . The simulation result also provides the loop delay $T_{\text {loop }}$ for this case, which is equal to $337.75 \mathrm{ps}$. Hence, the coarse counting result is equal to $\left(N_{1}+N_{2}\right) T_{\text {loop }}$ $=19927.25$ ps.

The 12-bit thermometer output result is shown in Figure 5.11. At the rising edge of the stop signal, the one-zero transition appears between the 2nd and 3rd stage. Confirming the start signal has passed 2 stages. Therefore, the fine counting result is $2 \times T_{L S B}=25.96 \mathrm{ps}$.

The total of the coarse and fine measurement results is calculated to be 19953.21 ps. For this mode, a single conversion has a 46.79 ps timing error.

The average power when the TDC operates on this mode is $13.84 \mathrm{~mW}$. 


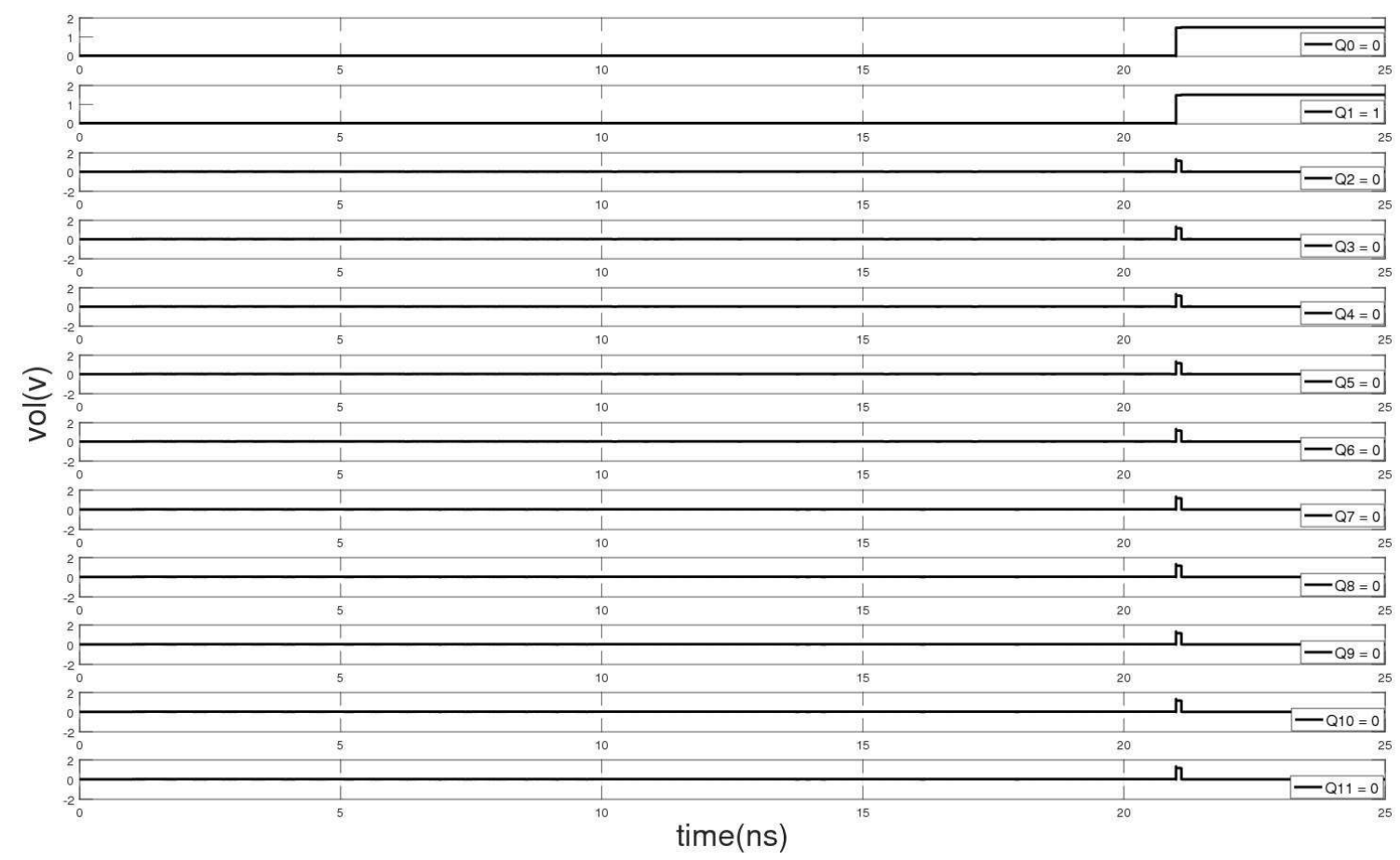

Fig. 5.11: The simulation results of the FFs. A 12-bit thermometer code is acquired. It contains 2 bits of logic 1 followed by 10 bits of logic 0 .

\subsubsection{The TDC Performance with 1 Load Activated}

The TDC operates with the maximum resolution $(14.3 \mathrm{ps})$ in this mode. This configuration is acquired by connecting only the first control voltage $V P 0$ to $0 \mathrm{~V}$. The same transient analysis is performed to probe the specific performance of the TDC core. Figure 5.12 shows the waveforms of the outputs Vout1 and Vout2 when the TDC works in this mode.

It confirms that the upper delay path oscillates for 27 cycles during the reference time interval. The lower signal propagates for 26 full cycles before the stop event. Therefore, the total number of loop cycles in this case is equal to $N_{1}+N_{2}$ which is 53 . The simulation result also provides the loop delay $T_{\text {loop }}$ for this case, which is equal to $371.8 \mathrm{ps}$. Hence, the coarse counting result is equal to $\left(N_{1}+N_{2}\right) T_{\text {loop }}=19705.4 \mathrm{ps}$. 


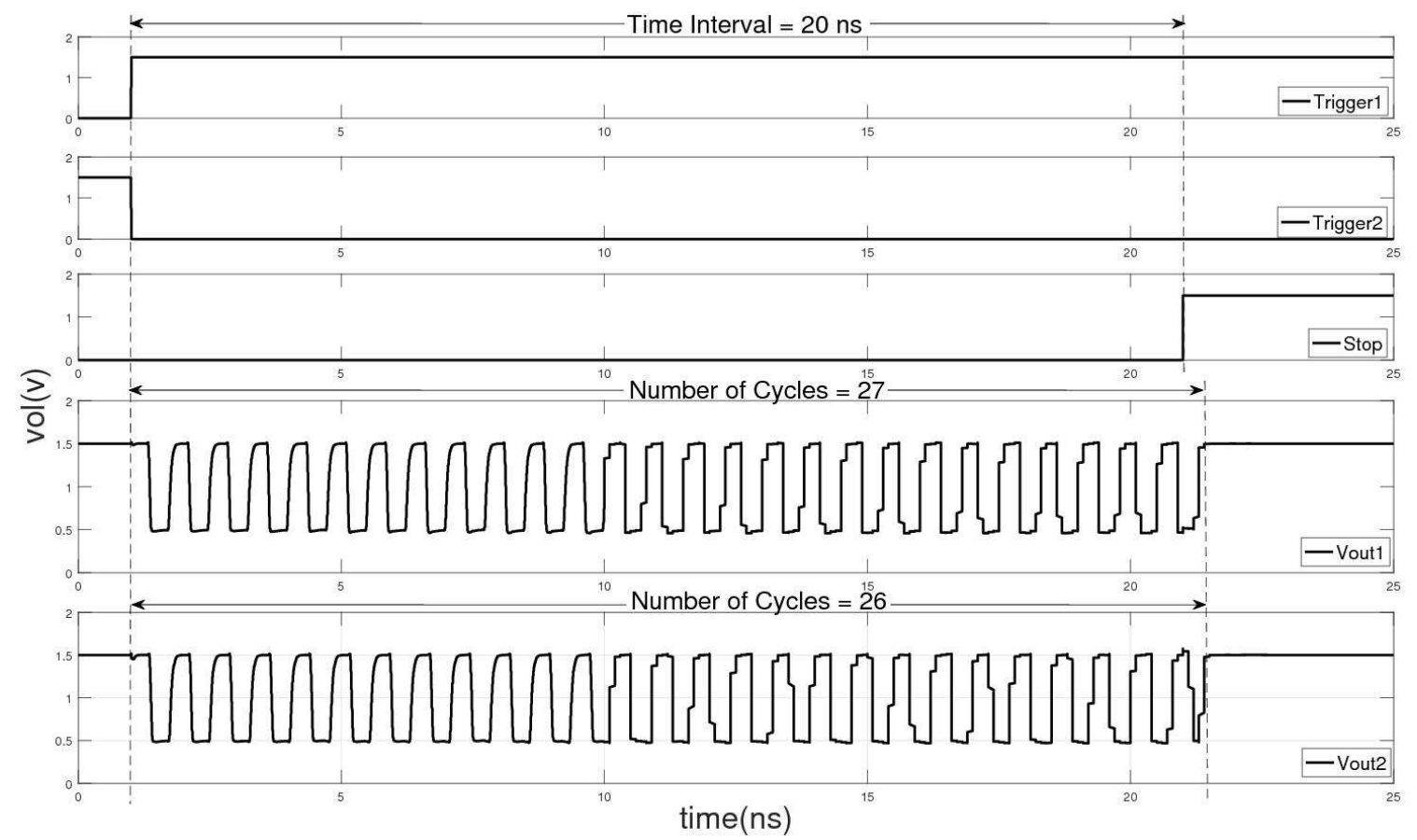

Fig. 5.12: The Simulation Result of a 20 ns Time Interval. The total number of cycles for the upper path is equal to 27 and the lower path is 26 .

The 12-bit thermometer output result is shown in Figure 5.13. At the rising edge of the stop signal, the one-zero transition appears between the 7th and 8th stage. It confirms that the start signal has passed 7 stages and therefore, the fine counting result is $7 \times T_{L S B}=100.1 \mathrm{ps}$.

The total of the coarse and fine measurement results is calculated to be 19805.5 ps. The TDC gives a 194.5 ps timing error, smaller than the reference time interval on this mode.

The average power when the TDC operates on this mode is $8.6 \mathrm{~mW}$. It consumes the minimum power since the TDC operates with its maximum resolution. 


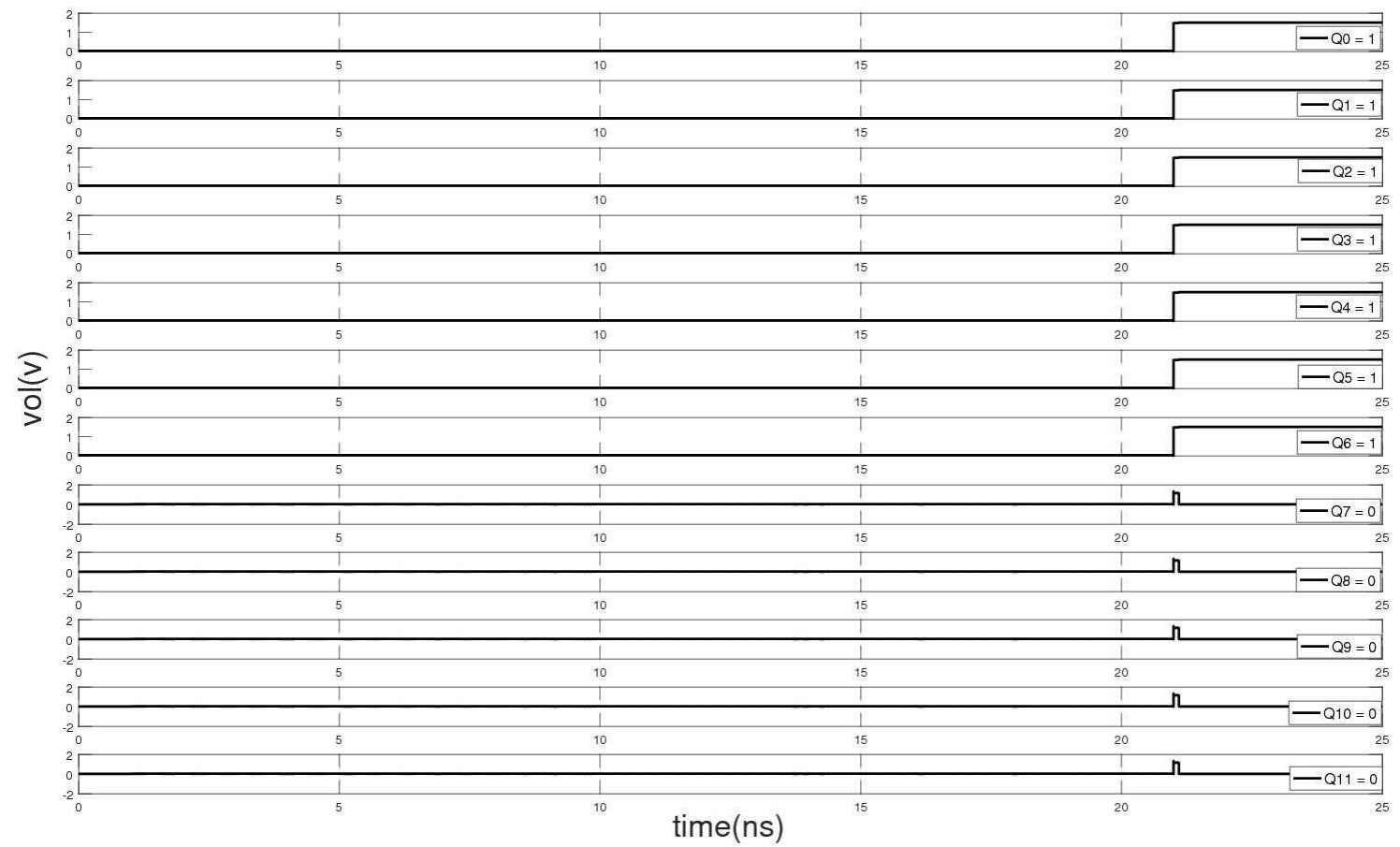

Fig. 5.13: The simulation results of the FFs. A 12-bit thermometer code is acquired. It contains 7 bits of logic 1 followed by 5 bits of logic 0 .

\subsection{Loop Counter Simulation Result}

This subsection is mainly focused on presenting the simulation result of the Loop Counter block. The output Vout1 from Figure 5.8 is chosen and injected into the Loop Counter block. The TDC core is operating on the second mode with a $10.83 \mathrm{ps}$ resolution. As shown before, the upper delay path oscillated 35 full cycles during the reference time interval (20 ns in this case).

The simulation result of the Loop Counter is shown in Figure 5.14. The TDC core and the Loop Counter use a synchronous stop signal. Therefore, the state freeze circuit which was attached to the counter outputs the counting result on the point of time at 21 ns. This result is a parallel 8-bit binary code originally.

The Loop Counter is designed to have 8 bits of output pins. Hence, each bit 


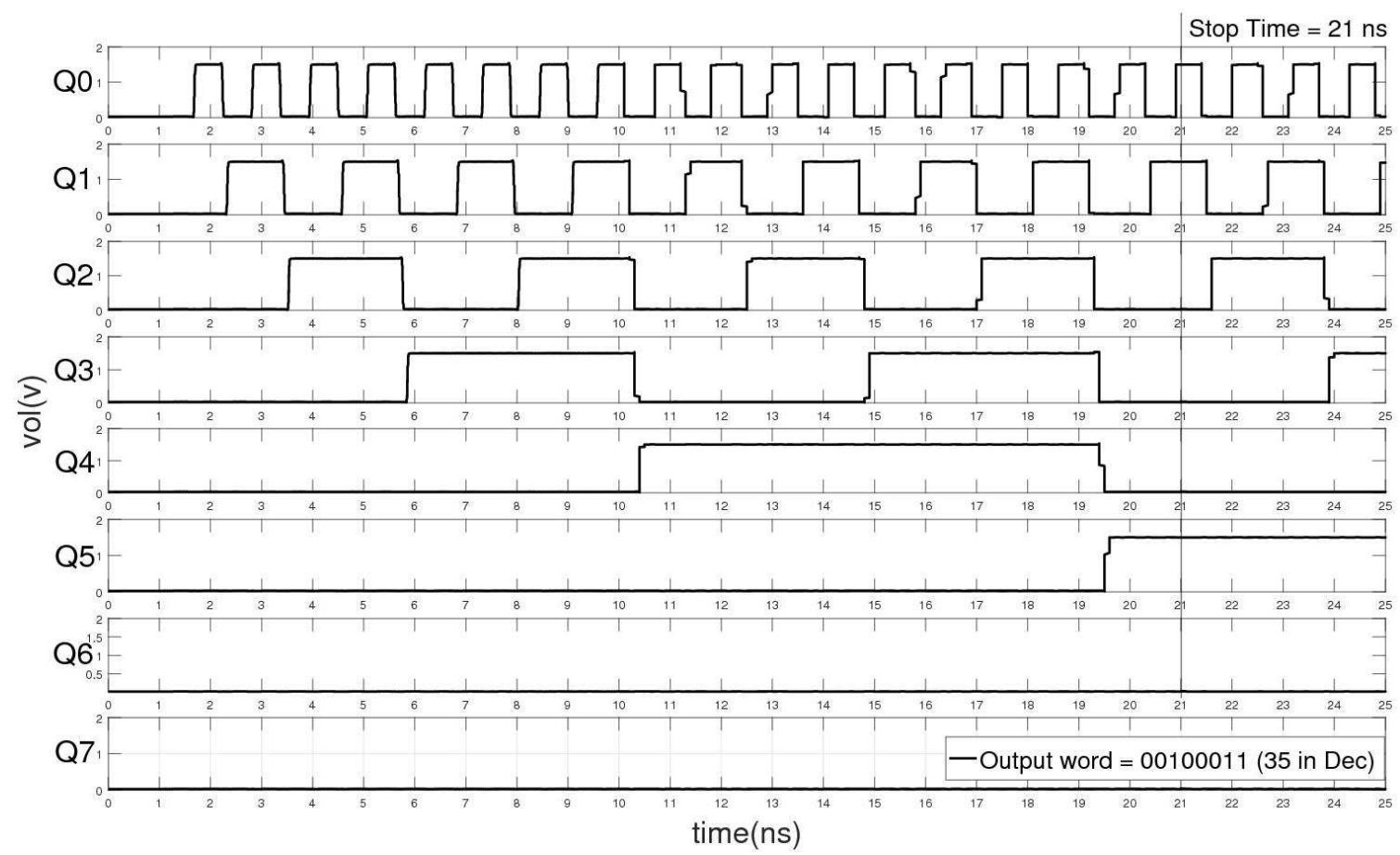

Fig. 5.14: The simulation result of the Loop Counter. The output binary code is 00100011 (Decimal 35) at the stop event which occurs at $21 \mathrm{~ns}$.

of the binary code corresponds to an output of the counter. Figure 5.14 gives the counting result of the Loop Count at $21 \mathrm{~ns}$. From $Q 0$ to $Q 7$, the parallel binary code is 00100011 which is equal to 35 in decimal.

Consequently, the simulation result proves that the Loop Counter works correctly. 


\subsection{PISO Shift Register and Driving Buffer Chain Simulation Result}

PISO Shift Register serves as the data form conversion device. This block is the peripheral circuit and a buffer chain is attached to it. Figure 5.15 shows the test bench used to perform the operation of this block.

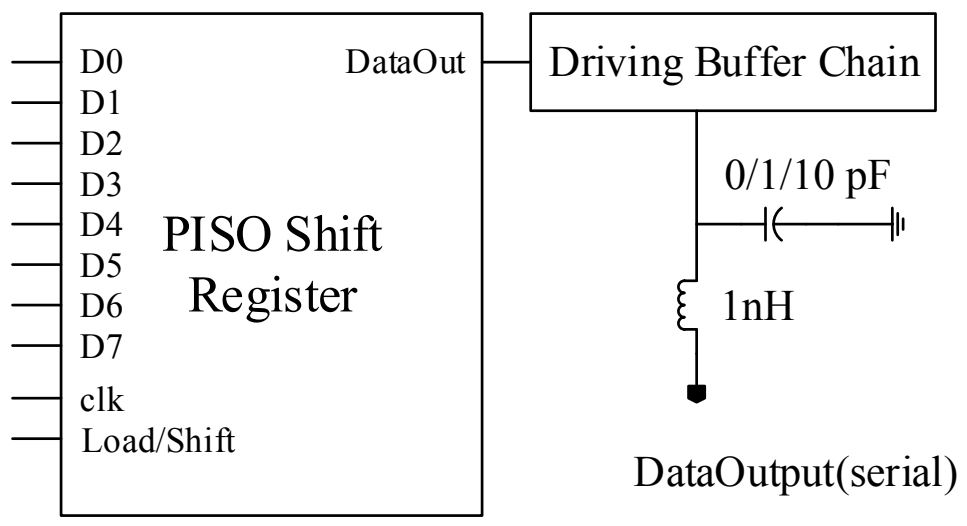

Fig. 5.15: The diagram of the test bench. A driving buffer chain is attached. A capacitor and an inductor is used to model the bond pad and the connections

This block is directly connected with the bond pad and a driving buffer chain is required to strengthen the output signal. A capacitor and an inductor is involved in order to model the bond pad and connection. The result from the last simulation (00100011) is applied to the input pins of the PISO Shift Register. The simulation result of this test bench is shown in Figure 5.16.

The first waveform is a $20 \mathrm{MHz}$ clock used to pump out the data. The clock frequency is acquired by simulating iteratively with different load capacitance. The load and shift process requires at least 8 clock cycles due to the 8-bit outputs of the counter. Thus, a 160 ns transient analysis is performed in order to show the complete data of the conversion. 


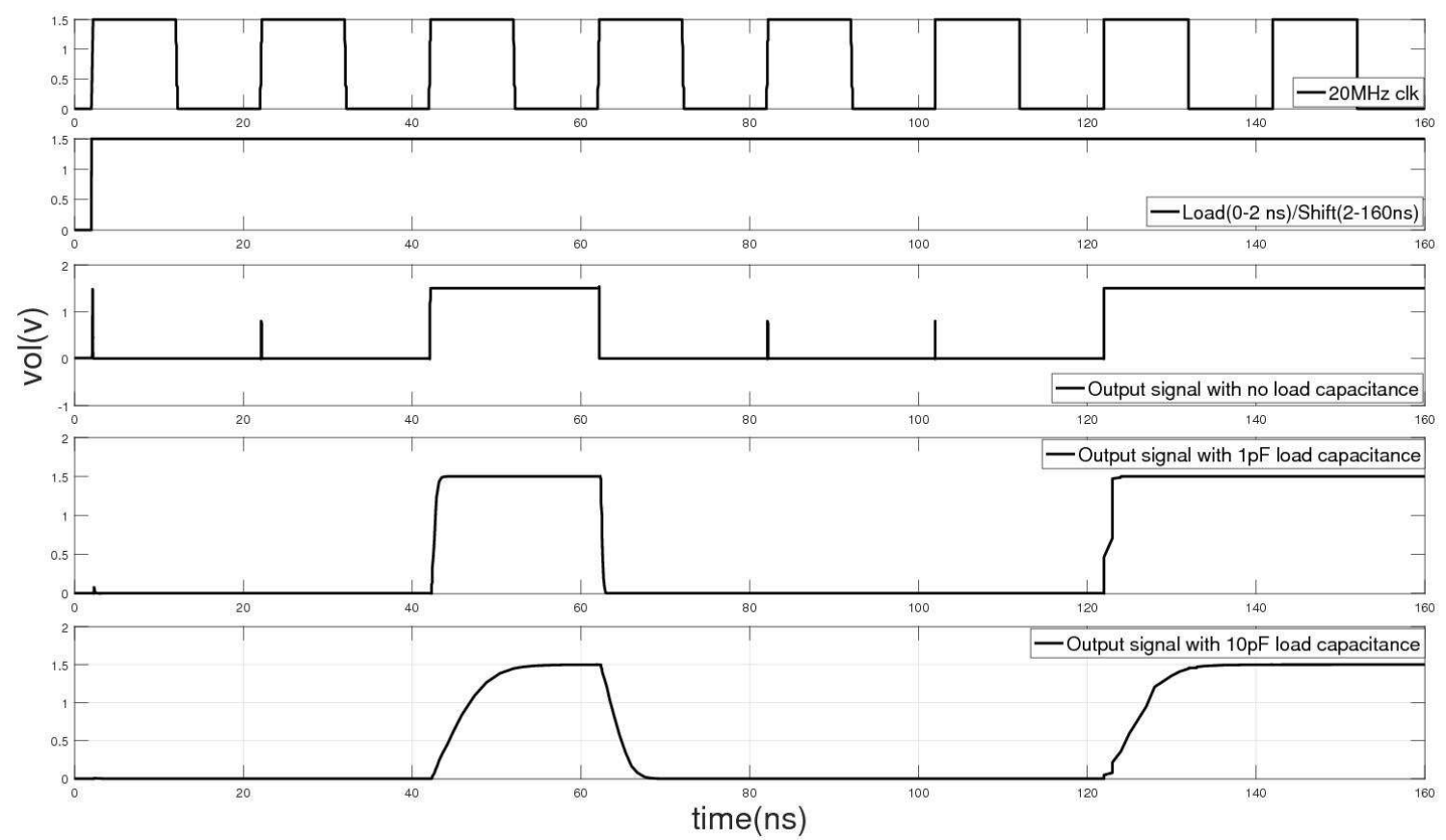

Fig. 5.16: The simulation result of the PISO Shift Register.

The second waveform is the control signal used to switch the PISO Shift Register from load to shift state. From the beginning of the simulation to $2 \mathrm{~ns}$, all data is loaded. Next, the control signal reaches high and enables the shifting process. Each clock cycle causes data to be shifted out one bit after another.

The third waveform is the output binary code in serial form. The simulation result is a perfect square wave without any load capacitance.

The 4th and 5th sub plots are the results of the simulation when the circuit is loaded an $1 p F$ or $10 p F$ capacitor. The register still works properly with a maximum $10 p F$ and $1 n H$ load.

As the Figure 5.16 shows, the output result is a serial binary code 00100011 from left to right. It proves that the parallel data has been correctly shifted into its corresponding serial form. 


\subsection{Summary of The Simulation Results}

As discussed previously, each TDC architecture has its own merits and drawbacks. For example, a Hierarchical TDC usually achieves the widest Dynamic Range and the smallest area. However, it is normally hard for it to obtain a small resolution. The other state-of-the-art architectures also gave better results for some of the parameters.

The Switchable MCML-Buffer based TDC has its unique multi-modes characteristic. The 4 PMOS loads unavoidably involve incidental area cost. However, 6 extra loads in each delay cell with the width of a few micrometers lead to a small area increment since this design includes only one TDC core measurement block. For the application where the TDC array is involved, the multi-step MCML cell may not be a good candidate. The TDC array normally consists of a large amount of TDC core blocks in order to perform multiple measurements simultaneously. Consequently, the area increment in each delay cell will accumulate and end up with a big area cost.

A comparison between the Switchable MCML-Buffer based TDC and the recent state-of-the-art architectures is presented in Table 5.2. 
Table 5.2: The recent state-of-the-art versus all modes of the Switchable MCML-Buffer based TDC

\begin{tabular}{|l|c|c|c|c|c|c|c|}
\hline & \multicolumn{3}{|c|}{ State-of-the-art } & \multicolumn{4}{c|}{ Operation Mode } \\
\hline Reference & {$[14]$} & {$[20]$} & {$[21]$} & 1 & 2 & 3 & 4 \\
\hline Type & GRO & Hierarchical & Time Amplification & \multicolumn{3}{|c|}{ Switchable MCML } \\
\hline Resolution $(\mathrm{pS})$ & 6 & 476 & $0.98(\mathrm{FB}) / 6.01(\mathrm{FF})$ & 8.24 & 10.83 & 12.98 & 14.3 \\
\hline DR (bit) & 11 & 15 & $10(\mathrm{FB}) / 3(\mathrm{FF})$ & \multicolumn{4}{|c|}{9} \\
\hline VDD $(\mathrm{V})$ & 1.5 & - & 1 & & \multicolumn{3}{|c|}{1.5} \\
\hline Power $(\mathrm{mW})$ & $3.3-31.5$ & 6.3 & $3(\mathrm{FB}) / 17.5(\mathrm{FF})$ & 19.29 & 15.26 & 13.84 & 8.6 \\
\hline Technology $(\mathrm{nm})$ & 130 & 350 & 65 & \multicolumn{4}{|c|}{130} \\
\hline Area $\left(\mathrm{mm}^{2}\right)$ & 0.04 & 0.16 & 0.02 & \multicolumn{4}{|c|}{0.06} \\
\hline
\end{tabular}


High priority parameters (resolution and power consumption) of each TDC type are selected and depicted in Figure 5.17 for the purpose of comparison.

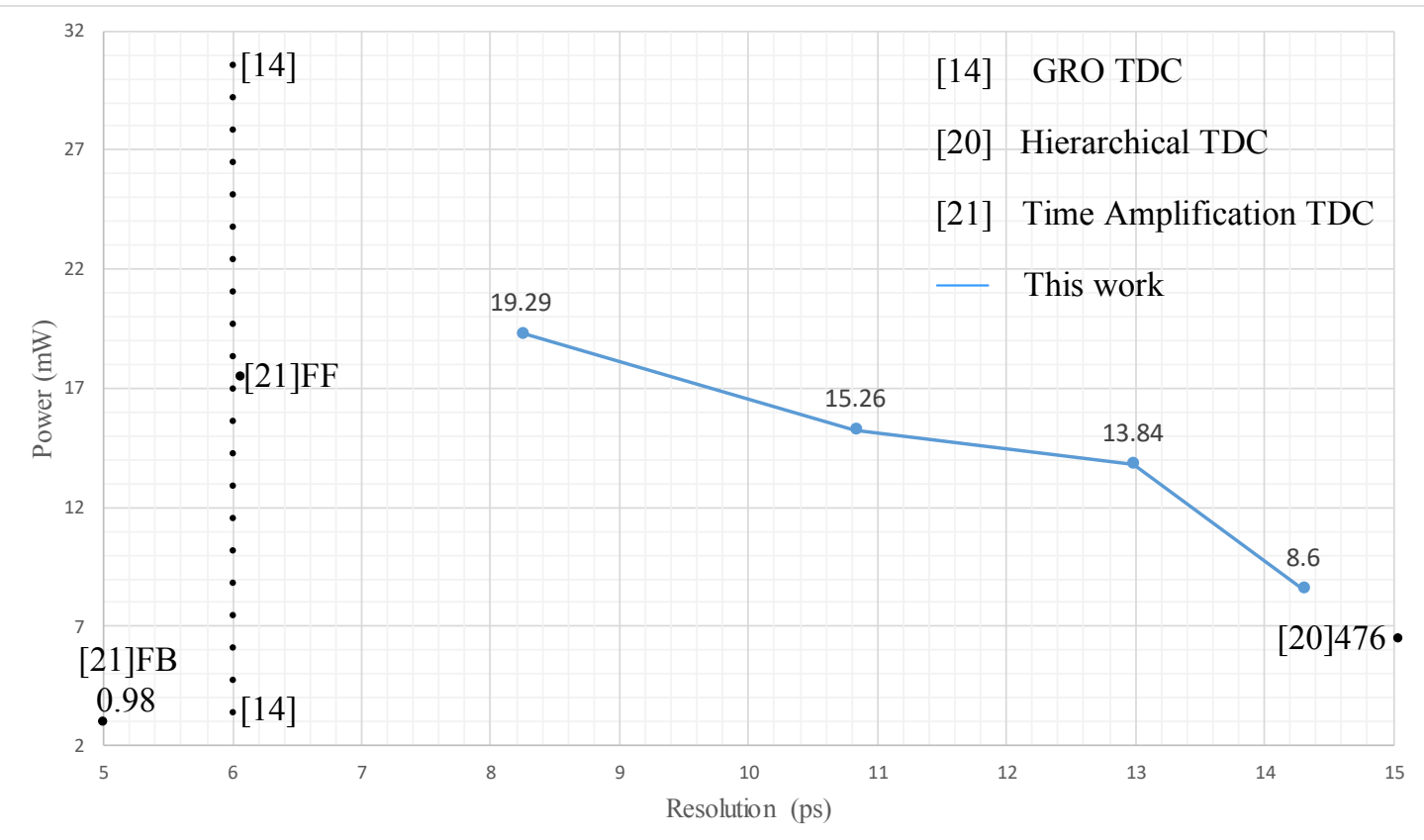

Fig. 5.17: Resolution and Power comparison.

The Time Amplification TDC has a Feedback mode with the resolution of 0.98 ps and the Hierarchical TDC in reference [20] obtains a resolution of $476 \mathrm{ps}$. It is hard to shown all of the corresponding points in one scale. Therefore, both of them are placed at the boundary of the comparison figure.

The reference [14] achieves a minimum resolution of $6 \mathrm{ps}$ which is slightly better than this design. In addition, it has a uncertain power swing from $3.3 \mathrm{~mW}$ to $31.5 \mathrm{~mW}$ which is depending on the time difference between input edges. This work quantizes a given time interval with four switchable resolutions and each corresponds to a certain power consumption. The GRO TDC achieves a better power consumption in short time interval quantization and this work has smaller power for long time interval measurement. 
Furthermore, a TDC that specifically designed for the TOF measurement usually requires a large quantization range. This is not only revealed by more digital bits output result but also the time interval that corresponds to each increment in the counting result. This means that even for the TDCs which have the same DR in the number of bits, the actual range detection capability is still different.

At last but not the least, this work has great potential in the aspect of optimizing the resolution and power consumption. For applications that do not require large measurement range, the device count of delay loop can be significantly reduce. This will achieves a great improvement at the parameters of area consumption and power. 


\section{Chapter 6}

\section{Conclusions and Future Work}

\subsection{Conclusions}

One of the major constraints of TDC design, while using standard digital cells, is that the resolution is limited mostly by the cell propagation delay. Furthermore, recent researches are largely focused on developing new architecture in order to achieve the sub-gated resolution. A TDC architecture that brings an effective enhancement on resolution normally cost extra die area or power consumption. Comparatively little focus has been placed on the fundamental delay cell implementation.

This thesis presented a new TDC using an optimized MCML-Buffer with 4-step selectable delay. The key idea of the design is that the delay cell is inherently adjustable due to the inclusion of the four selectively switched active PMOS loads, which in practise influence the voltage swing at the output of the cell, thereby adjusting the propagation delay through the cell.

In addition, the complexity of the counting devices are also reduced. This is achieved by replacing the conventional dual-edge triggered loop counter with a pair of single edge triggered counter. This configuration benefits from the differential characteristic of the MCML gates. 
A full chip layout is constructed and the chip has been manufactured by CMC. System and circuit level design and implementation were presented in detail to introduce the TDC prototype. Unfortunately, the chip prototype will be measured by another student at a later date due to time constrains. My task was to design and optimize the novel TDC architecture. Furthermore, the circuit has been justified according to the simulation results. 


\subsection{Future Work}

Based on the results of this thesis, the following future work can be undertaken:

- Combining the coarse counting results with the fine counting results:

An evaluation circuit that combines the coarse counting results (8-bit binary codes) with the fine counting results (12-bit thermometer code) will bring completion to this design and significantly improve the efficiency of the simulation and test.

- Extending the operation modes of the MCML-Buffer based TDC circuit:

Currently, the Switchable MCML-Buffer based TDC has four operation modes and covers four different resolutions. An extended PMOS loads network will gives the delay elements the ability of operating on extra modes

- The manufactured integrated circuit will be measured and compared to simulated results. 


\section{List of References}

[1] J. Markoff, "Google cars drive themselves, in traffic," The New York Times, vol. 10, no. A1, p. 9, 2010.

[2] J. Levinson, M. Montemerlo, and S. Thrun, "Map-based precision vehicle localization in urban environments.," in Robotics: Science and Systems, vol. 4, p. 1, Citeseer, 2007.

[3] J. Levinson and S. Thrun, "Robust vehicle localization in urban environments using probabilistic maps," in Robotics and Automation (ICRA), 2010 IEEE International Conference on, pp. 4372-4378, IEEE, May. 2010.

[4] R. B. Rusu and S. Cousins, "3d is here: Point cloud library (pcl)," in Robotics and Automation (ICRA), 2011 IEEE International Conference on, pp. 1-4, IEEE, 2011.

[5] E. Ackerman, "Lidar that will make self-driving cars affordable [news]," IEEE Spectrum, vol. 53, no. 10, pp. 14-14, 2016.

[6] C. Petersen, ":: peeto. net-technology," 2017.

[7] R. W. Wolcott and R. M. Eustice, "Visual localization within lidar maps for automated urban driving," in Intelligent Robots and Systems (IROS 2014), 2014 IEEE/RSJ International Conference on, pp. 176-183, IEEE, Sep. 2014.

[8] S. Henzler, Time-to-digital converters, vol. 29. Springer Science \& Business Media, 2010.

[9] K. Niitsu, M. Sakurai, N. Harigai, T. J. Yamaguchi, and H. Kobayashi, "Cmos circuits to measure timing jitter using a self-referenced clock and a cascaded time difference amplifier with duty-cycle compensation," IEEE Journal of Solid-State Circuits, vol. 47, pp. 2701-2710, Nov. 2012. 
[10] B. Dehlaghi, S. Magierowski, and L. Belostotski, "Highly-linear time-difference amplifier with low sensitivity to process variations," Electronics letters, vol. 47, pp. 743-745, Jun. 2011.

[11] R. B. Staszewski, D. Leipold, C.-M. Hung, and P. T. Balsara, "Tdc-based frequency synthesizer for wireless applications," in Radio Frequency Integrated Circuits (RFIC) Symposium, 2004. Digest of Papers. 2004 IEEE, pp. 215-218, IEEE, 2004.

[12] L. Xiao, Z. Xiao, Z. Qian, Y. Jiang, T. Huang, H. Tian, and E. F. Young, "Local clock skew minimization using blockage-aware mixed tree-mesh clock network," in Proceedings of the International Conference on Computer-Aided Design, pp. 458-462, IEEE Press, Nov. 2010.

[13] J.-L. Tsai and L. Zhang, "Statistical timing analysis driven post-silicon-tunable clock-tree synthesis," in Proceedings of the 2005 IEEE/ACM International conference on Computer-aided design, pp. 575-581, IEEE Computer Society, Nov. 2005.

[14] M. Z. Straayer and M. H. Perrott, "A multi-path gated ring oscillator tdc with first-order noise shaping," IEEE Journal of Solid-State Circuits, vol. 44, pp. 1089-1098, Apr. 2009.

[15] A. S. Yousif and J. W. Haslett, "A fine resolution tdc architecture for next generation pet imaging," IEEE Transactions on nuclear science, vol. 54, pp. 1574-1582, Oct. 2007.

[16] K. Murali, S. Sinha, and W. L. Ditto, "Implementation of nor gate by a chaotic chua's circuit," International Journal of Bifurcation and Chaos, vol. 13, pp. 26692672, Sep. 2003.

[17] B. Markovic, S. Tisa, F. A. Villa, A. Tosi, and F. Zappa, "A high-linearity, 17 ps precision time-to-digital converter based on a single-stage vernier delay loop fine interpolation," IEEE Transactions on Circuits and Systems I: Regular Papers, vol. 60, pp. 557-569, Mar. 2013.

[18] J. Yu, F. F. Dai, and R. C. Jaeger, "A 12-bit vernier ring time-to-digital converter in 0.13 cmos technology," IEEE journal of solid-state circuits, vol. 45, pp. 830842, Apr. 2010. 
[19] O. Musa and M. Shams, "An efficient delay model for mos current-mode logic automated design and optimization," IEEE Transactions on Circuits and Systems I: Regular Papers, vol. 57, pp. 2041-2052, Aug. 2010.

[20] J. Wu, Q. Jiang, K. Song, L. Zheng, D. Sun, and W. Sun, "Implementation of a high-precision and wide-range time-to-digital converter with three-level conversion scheme," IEEE Transactions on Circuits and Systems II: Express Briefs, vol. 64, pp. 181-185, Feb. 2017.

[21] J.-C. Lai and T.-Y. Hsu, "Cost-effective time-to-digital converter using timeresidue feedback," IEEE Transactions on Industrial Electronics, vol. 64, pp. 4690-4700, Jun. 2017.

[22] J. Kong, L. Siek, and C.-L. Kok, "A 9-bit body-biased vernier ring time-to-digital converter in $65 \mathrm{~nm}$ cmos technology," in Circuits and Systems (ISCAS), 2015 IEEE International Symposium on, pp. 1650-1653, IEEE, May. 2015.

[23] A.-J. Annema, B. Nauta, R. Van Langevelde, and H. Tuinhout, "Analog circuits in ultra-deep-submicron cmos," IEEE Journal of Solid-State Circuits, vol. 40, pp. 132-143, Jan. 2005.

[24] M. Usama and T. Kwasniewski, "Design and comparison of cmos current mode logic latches," in Circuits and Systems, 2004. ISCAS'04. Proceedings of the 2004 International Symposium on, vol. 4, pp. IV-353, IEEE, 2004.

[25] H. Hassan, M. Anis, and M. Elmasry, "Mos current mode circuits: analysis, design, and variability," IEEE Transactions on Very Large Scale Integration (VLSI) Systems, vol. 13, pp. 885-898, Aug. 2005.

[26] H. Hassan, M. Anis, and M. Elmasry, "Design and optimization of mos current mode logic for parameter variations," INTEGRATION, the VLSI journal, vol. 38, pp. 417-437, Apr. 2005.

[27] M. Alioto and G. Palumbo, "Design strategies for source coupled logic gates," IEEE Transactions on Circuits and Systems I: Fundamental Theory and Applications, vol. 50, pp. 640-654, May. 2003.

[28] Y. Cheng and C. Hu, MOSFET modeling \& BSIM3 users guide. Springer Science \& Business Media, 1999. 
[29] S. Henzler, S. Koeppe, D. Lorenz, W. Kamp, R. Kuenemund, and D. SchmittLandsiedel, "A local passive time interpolation concept for variation-tolerant high-resolution time-to-digital conversion," IEEE Journal of Solid-State Circuits, vol. 43, pp. 1666-1676, Jul. 2008.

[30] P. Chen, C.-C. Chen, C.-C. Tsai, and W.-F. Lu, "A time-to-digital-converterbased cmos smart temperature sensor," IEEE Journal of Solid-State Circuits, vol. 40, pp. 1642-1648, Aug. 2005.

[31] Y. Liu, U. Vollenbruch, Y. Chen, C. Wicpalek, L. Maurer, Z. Boos, and R. Weigel, "Multi-stage pulse shrinking time-to-digital converter for time interval measurements," in Microwave Integrated Circuit Conference, 200\%. EuMIC 200\%. European, pp. 267-270, IEEE, 2007.

[32] M. Lee and A. A. Abidi, "A 9 b, 1.25 ps resolution coarse-fine time-to-digital converter in $90 \mathrm{~nm}$ cmos that amplifies a time residue," IEEE Journal of solidstate circuits, vol. 43, pp. 769-777, Apr. 2008.

[33] S.-H. Chen and M.-B. Lin, "A synthesizable architecture of all-digital cyclic tdcs," IEICE Electronics Express, vol. 11, no. 20, pp. 20140875-20140875, 2014.

[34] M. M. Mano, C. R. Kime, T. Martin, et al., Logic and computer design fundamentals, vol. 3. Prentice Hall, 2008.

[35] N. Nedovic and V. G. Oklobdzija, "Dual-edge triggered storage elements and clocking strategy for low-power systems," IEEE Transactions on Very Large Scale Integration (VLSI) Systems, vol. 13, pp. 577-590, May. 2005.

[36] B. S. Cherkauer and E. G. Friedman, "Unification of speed, power, area, and reliability in cmos tapered buffer design," in Circuits and Systems, 1994. ISCAS'94., 1994 IEEE International Symposium on, vol. 4, pp. 111-114, IEEE, 1994.

[37] S. Milicevic and L. MacEachern, "Frequency dividers implementing custom cells with resistor tail bias," in Signals, Systems and Electronics, 200\%. ISSSE'0\%. International Symposium on, pp. 493-496, IEEE, 2007.

[38] K. J. Kuhn, "Reducing variation in advanced logic technologies: Approaches to process and design for manufacturability of nanoscale cmos," in Electron Devices Meeting, 200\%. IEDM 200\%. IEEE International, pp. 471-474, IEEE, 2007. 
[39] J. Rice, "An analysis of several proposals for reversible latches," in Advances and Innovations in Systems, Computing Sciences and Software Engineering, pp. 203206, Springer, 2007.

[40] P. J. Restle, T. G. McNamara, D. A. Webber, P. J. Camporese, K. F. Eng, K. A. Jenkins, D. H. Allen, M. J. Rohn, M. P. Quaranta, D. W. Boerstler, et al., "A clock distribution network for microprocessors," IEEE Journal of Solid-State Circuits, vol. 36, pp. 792-799, May. 2001.

[41] J. Richardson, R. Walker, L. Grant, D. Stoppa, F. Borghetti, E. Charbon, M. Gersbach, and R. K. Henderson, "A 32× 32 50ps resolution 10 bit time to digital converter array in 130nm cmos for time correlated imaging," in Custom Integrated Circuits Conference, 2009. CICC'09. IEEE, pp. 77-80, IEEE, 2009.

[42] T. Charania, A. Opal, and M. Sachdev, "Analysis and design of on-chip decoupling capacitors," IEEE Transactions on Very Large Scale Integration (VLSI) Systems, vol. 21, pp. 648-658, Apr. 2013. 